\title{
Evoluzione storica delle misure orarie in Italia
}

(Historical evolution of the horary measurements in Italy)

Suoi riflessi nello studio dei fenomeni geofisici storici, $\mathrm{e}$ in particolare nelle catalogazioni di eventi sismici.

\section{P. Dominici* - L. MARCElli*}

Ricevuta il 20 Iuglio, 1979

\section{Abstract}

The present paper represents a research on the historical evolution, in Italy, regarding the measurement of hours during the day. A few cosmographical theoric recalls are offered to clarify how the problem should be considered from the astronomical viewpoint. A review is then made of the different chronometrical scales adopted in Italy, since Roman times; then, later, in the High and Low Medieval periods down to modern and contemporary times. All the different chronometrical scales adopted, in their historical evolution, are investigated: temporary, equi. noctial, Italian hours, true local, mean local, civil, time hours a.m. and p.m., mean Rome, Mean Central European, Universal and finally Univer. sally Coordinate, time hours; the intermittent adoption of legal summer time, is recalled.

The importance of the problem is evidenced on account of the reper. cussion that an inaccurate interpretation of the different time measures given in historical times can have on geophysical events, and especially

(*) Istituto Nazionale di Geofisica, Roma. 
on an indexing of seismic events occurring in epochs prior to the existence of radiosignalled time.

To illustrate the research, reference is made to the classical treatise of Baratta "I terremoti d'ltalia» (ed. 1901): possibility of alterations of the fina lresults can be observed unless special attention is given to the original chronicles. The alterations can even produce a date change or a repetition, in the same catalogue, of the same event.

Reliability criteria are suggested for hour data in function of the cpoch of the event, as well as for the chronometrical scale employed in evidencing said event.

In appendix some passages drawn from a study made by Serpieri on the Rimini earthquake of March 18, 1875 that confirm tre difficulties evidence are reported.

Some tables are also reported:

a table that gives the start of the clay for the calculation of Italian hours at different latitude sand at different days of the year, prior to and after the Gregorian reformation of the calendar, as well as the corresponding time equations (App. E);

a table that gives the geoghaphical coordinates of the Italian provincial capitals with the longitudes (referred to Greenwich and Rome) expressed in time (App. D);

a table showing time periods where legal (summer) time was adopted instead of solar time (App. B);

a table of intercalar seconds of Universal Coordinate time (App. A).

\section{RIASSUNTO}

Il presente lavoro vuole essere una ricerca sull'evoluzione storica che ha subito, in Italia, il modo di misurare le ore durante la giornata.

Alcuni richiami teorici di cosmografia vengono dati per chiarire come dovrebbe essere impostato il problema dal punto di vista astronomico.

Si passa indi all'esposizione delle varie scale cronometriche adottate in Italia a partire fin dal periodo romano; poi, successivamente, nell'alto e basso medioevo fino all'età moderna e contemporanea.

Vengono esaminate, nella loro evoluzione storica, tutte le varie scale cronometriche adottate: ore temporarie, ore equinoziali, ore italiane, ore in tempo vero locale, in tempo medio locale, in tempo civile, in ore a.m. e p.m., in tempo medio di Roma, in Tempo Medio Europa Centrale, in Tempo Universale e infine in Tempo Universale Coordinato; viene ricordata l'adozione saltuaria delle ore legali estive.

Si evidenzia l'importanza del problema per la ripercussione che una interpretazione non accurata delle varie misure orarie date in tempi 
storici può avere su eventi geofisici, e in particolare su una catalogazione di eventi sismici avvenuti in epoche precedenti l'avvento dei radiosegnali orari.

Per illustrare la ricerca viene fatto riferimento a ltrattato classico del Baratta "I terremoti d'Italia " (ed. 1901): si riscontra la possibilità di alterazioni dei risultati finali ove non si ponga una debita attenzione alle cronache originali. Le alterazioni possono raggiungere perfino un cambiamento di data, o una ripetizione, nello stesso catalogo, del medesimo evento.

Vengono suggeriti dei criteri di attendibilità delle misure orarie in funzione dell'epoca cui risale l'evento, e del tipo di scala cronometrica usata per la sua segnalazione.

In appendice sono riportate, oltre ad alcuni passi tratti da uno studio del Serpieri sul terremoto riminese del 18 marzo 1875 che confermano le difficoltà segnalate, anche varie tabelle:

una tabella che dà l'inizio del giorno per il computo delle ore ita. liane alle varie latitudini e nei vari giorni dell'anno, prima e dopo la riforma gregoriana del calendario, nonché le corrispondenti equazioni del tempo (App. E);

una tabella che dà le coordinate geografiche dei capoluoghi di provincia italiani con le longitudini (rispetto a Greenwich e rispetto a Roma) espresse in tempo (App. D);

una tabella indicante i periodi di tempo nei quali è stata adottata l'ora legale (estiva) in luogo di quella solare (App. B);

una labella indicante l'introduzione dei secondi intercalari per la esatta conversione delle misure di tempo in T.U.C. (App. A).

\section{INTRODUZIONE}

Nel lavoro intrapreso recentemente dall'Istituto Nazionale di Geofisica per redigere un catalogo completo degli eventi sismici che hanno interessato il territorio italiano dall'inizio dell'era cristiana a tutt'oggi, ci si è trovati, impensatamente, di fronte a notevoli difficoltà interpretative circa la valutazione del "tempo origine" che, come si sa, è di basilare importanza per ogni studio che si voglia intraprendere su un qualsiasi terremoto.

Per la catalogazione sismica - ma l'osservazione vale, naturalmente, per ogni descrizione "storica " di fenomeni geofisici si hanno a disposizione riferimenti temporali la cui accuratezza diminuisce via via che si risale nel passato; da un certo punto in poi - sempre procedendo a ritroso, ma più vicino al presente 
di quanto non si pensi - alle ben identificabili cause di imprecisione che sono legate alla povertà dei mezzi e dei metodi di osservazione s'aggiungono insidiose incertezze che sono legate all'interpretazione delle misure temporali riportate nelle relazioni tecnico-scientifiche e nelle cronache della vita civile.

Nell'uso geofisico odierno la relatività temporale degli eventi è valutata riportando tutte le ascisse temporali al cosiddetto " tempo universale ": è quindi evidente che non corrette trasformazioni in tale tempo dei tempi ricavati da vecchie relazioni e da cronache antiche (i quali fino a circa un secolo fa erano tutti "tempi locali ", di varia natura) possono oggi portare a notevoli distorsioni interpretative degli eventi, con il grave rischio limite di portare in conto più di una volta il medesimo evento se è riferito da fonti storiche diverse ad ascisse temporali apparentemente diverse.

Di fronte a questa situazione, gli autori del presente lavoro hanno ritenuto utile presentare una rassegna critica delle cosiddette "scale cronometriche", cioè delle scale di misura con cui è stato valutato nei secoli in Italia lo scorrere del tempo nell'arco della gionata, con qualche richiamo alle implicazioni riguardanti la data, cioè alle cosiddette "scale cronologiche".

Nel procedere del lavoro, essi sono stati colpiti in particolare da due fatti, e cioè dalla scarsità di studi sull'argomento e dalla rozzezza delle misure temporali del passato, protrattasi anche dopo l'introduzione - sul finire dell'altro secolo - di un'ora unica in ogni singola nazione.

Relativamente a quest'ultima circostanza, non va dimenticato che prima dell'introduzione di vaste reti telegrafiche e poi anche telefoniche $\left({ }^{*}\right)$, e di ferrovie a lungo percorso $\left({ }^{* *}\right)$, la vita scorreva in ambito strettamente locale e, poiché non esistevano comunicazioni rapide, non si poneva in pratica il problema della correlazione temporale fra luoghi diversi. Questo serve a rendersi conto del perché le misure orarie del passato, anche prossimo, fossero date tutte in tempo locale.

(*) In Italia, all'incirca dal 1850 per le reti telegrafiche, e dal 1881 per quelle telefoniche.

$\left.{ }^{* *}\right)$ In Italia, ferrovie interregionali si ebbero subito dopo il 1861. 
Anche quando sarebbe stato possibile, con metodi astronomici, collegare tutti gli orologi di una regione in modo che indicassero la stessa ora, mancava tuttavia una qualunque necessità pratica che giustificasse una cosa del genere, molto onerosa per i mezzi tecnici del passato; ore "nazionali " (e come tali - è da presumere - sufficientemente controllate e perciò attendibili) furono introdotte (anche nei paesi che, come la Gran Bretagna e la Francia si costituirono in nazione relativamente presto) in tempi piuttosto vicini a noi, e precisamente quando, nella seconda metà del secolo scorso, si manifestò la necessità di gestire reti telegrafiche e ferroviarie nazionali e internazionali. Prima che ciò accadesse, per tutte le esigenze della vita individuale e collettiva veniva spontaneo regolarsi sul corso diurno locale del Sole, cioè sullo scorrere di quello che propriamente si chiama " tempo solare ", scandito nella remota antichità da determinate consuetudini religiose e civili (come la celebrazione di particolari riti o processi, ecc., in determinati momenti del giorno solare) e più tardi, nell'Occidente cristiano, scandito dal suono delle campane delle chiese.

Per moltissimo tempo, scarso interesse pratico ha avuto, genericamente parlando, l'accurata determinazione dello scorrere del giorno: espressioni quali "all'alba ", "al mattino presto", " intorno al mezzodì ", "verso il tramonto " e, più avanti, misure orarie riferite alle ore, mezz'ore e quarti d'ora battuti dagli orologi civici erano sufficienti per la cronachistica dell'epoca (è da notare che sono ritenute sufficientemente accurate anche secondo i criteri storiografici moderni). Ciò spiega sia la povertà delle misure orarie tramandateci dalle cronache, sia il relativo disinteresse che gli storici - e sorprendentemente anche astronomi e fisici - hanno dimostrato per la cronometria antica. Ci auguriamo che, sul nostro esempio, altri studiosi, di estrazione diversa dalla nostra, siano tentati di migliorare, con ben maggiore competenza quanto al reperimento delle fonti, la conoscenza - sotto questo aspetto - del modo di vivere degli antichi abitanti del nostro paese.

Per quanto ci riguarda, e indulgendo a un certo amore per la completezza, riteniamo opportuno premettere alla descrizione delle scale cronometriche che sono state usate via via in Italia 
— argomento che costituirà la seconda parte del lavoro - una prima parte in cui si richiamano organicamente nozioni generalmente ben note ai cultori di scienze geofisiche sulle scale cronometriche definite dapprima soltanto su base astronomica e poi, venendo ai nostri tempi, su base astronomica e fisica. A queste due parti seguirà una terza parte, in cui verranno discussi, a titolo di esempio abbastanza generale, i criteri di interpretazione di misure orarie riferentisi a eventi sismici. 


\section{PARTE PRIMA}

Scale di tempo astronomiche e astronomico-fisiche: dal tempo solare vero al tempo universale coordinato.

Tutte le scale di tempo con cui l'uomo ha regolato le sue attività e di cui si è servito per descrivere i fenomeni naturali hanno sempre fatto riferimento, sin dalla più remota antichità, al moto di cui il Sole sembra essere animato sulla sfera celeste in conseguenza dei moti effettivi della Terra, e principalmente in conseguenza del moto intrinseco di rotazione e del moto di rivoluzione intorno al Sole.

\section{Richiami di cosmografia.}

Ricordiamo che la sfera celeste è la sfera ideale avente il centro nel centro $\mathbf{C}$ della Terra, sulla quale si proiettano gli astri per un osservatore situato nel generico punto $\mathbf{P}$ della superficie terrestre. Nella fig. 1 sono indicati i principali elementi di tale sfera; in particolare, $\mathbf{Z}$ è lo zenit di $\mathbf{P}$ (traccia della verticale $\mathbf{v}$ ), $\mathbf{B}$ è il polo celeste nord (proiezione da $\mathbf{C}$ del polo nord terrestre) e il piano della figura è il piano del "cerchio meridiano" (o semplicemente "meridiano") di $\mathbf{P}$ (intersezione della sfera celeste con il piano per $\mathbf{P}$ e per l'asse della rotazione terrestre), essendo E l'intersezione dell'equatore celeste (proiezione da $\mathbf{C}$ dell'equatore terrestre) con il "meridiano superiore" di $\mathbf{P}$, cioè con il semicerchio meridiano (NBZS) che si svolge al di sopra dell'orizzonte.

In conseguenza della rotazione terrestre, che avviene da ovest verso est ed è - in prima, buona approssimazione - uniforme, per l'osservatore in $\mathbf{P}$ è come se la sfera celeste rotasse uniformente con la stessa velocità angolare della Terra ma in verso opposto, cioè da est verso ovest, e in tale verso sembrano muoversi gli astri sulla sfera medesima. In particolare, il Sole, s, sembra percorrere sulla sfera un parallelo, p, di cui i punti notevoli sono il punto $\mathbf{A}$ in cui l'astro si leva sull'orizzonte (alba) e quello $\mathbf{T}$ (tramonto) in cui esso passa al disot to dell'orizzonte, nonché i punti $\mathbf{M}$ (mezzogiorno) ed $\mathbf{m}$ (mezzanotte) in cui il Sole passa al meridiano superiore (culminazione superiore) e inferiore (culminazione inferiore), rispettivamente.

Nell'intervallo di tempo fra l'alba e il tramonto si ha l'« arco diurno", in cui il Sole raggiunge la massima altezza sull'orizzonte $\mathbf{h}(0$, se si vuole, la minima distanza zenitale $\%$ ) al mezzogiorno $\mathbf{M}$; fra il tramonto e l'alba si ha l'« arco notturno ", in cui il Sole raggiunge la massima depressione 
sot to l'orizzonte (e quindi la massima distanza zenitale) alla mezzanotte $\mathbf{m}$.

Correntemente, gli archi diurno e notturno sono chiamati «giorno * e "notte" rispettivamente; in astronomia, propriamente, si chiama però "giorno l'intervallo di tempo fra due passaggi successivi del Sole al meridiano superiore. Tale intervallo di tempo è chiamato specificamente "giorno solare" per distinguerlo dal "giorno siderale", che intercorre fra due successive culminazioni superiori di una medesima stella ed è, con ottima approssimazione, il periodo della rotazione terrestre $\left(^{*}\right)$. Va da sć che, essendo l'alternarsi del giorno e della notte legato non al giorno siderale ma a quello solare, è a quest'ultimo che fanno riferimento le scale cronometriche.

In conseguenza della rivoluzione della Terra intorno al Sole, que. st'ultimo descrive in un anno, spostandosi lungo le costellazioni dello Zodiaco, un cerchio massimo della sfera celeste, l'eclittica, inclinato di un angolo $\varepsilon$ pari a circa $23^{\circ} 27^{\prime}$ sull'equatore. Come ben appare dalla figura 1 , spostandosi il Sole sull'eclittica in virtù del moto annuo terrestre (circa $1^{\circ}$ al giorno), ogni giorno esso descrive, a causa del moto diurno terrestre, un parallelo celeste diverso da quello del giorno precedente, talché di giorno in giorno variano sia la durata dell'arco diurno sia quella dell'arco notturno.

Una situazione particolare si ha nelle epoche in cui il Sole passa per i due punti della sfera celeste in cui s'incontrano equatore ed eclittica. Il parallelo descritto dal Sole coincide allora con l'equatore, che, come cerchio massimo, è bisecato dall'altro cerchio massimo costituito dall'orizzonte del luogo, per modo che arco diurno e arco notturno sono uguali fra loro su tutta la Terra: di qui la denominazione di "punti equinoziali". Di questi, si chiama "punta vernale", $\gamma$, quello che corrisponde all'equinozio di primavera.

L'alternarsi delle stagioni è legato al ritorno del Sole al punto vernale, che avviene in un intervallo di tempo di circa 365 giorni e un quarto, detto "anno tropico"; tutti i calendari e i computi cronologici sono basati su tale intervallo di tempo.

L'anno tropico è leggermente minore (per $20 \mathrm{~m} 24 \mathrm{~s}$ circa) del periodo della rivoluzione terrestre, detto "anno siderale" in quanto ragguagliabile all'intervallo di tempo che il Sole impiega per tornare in congiunzione con una medesima stella.

Per quanto riguarda la durata dell'arco diurno e di quello notturno, situazioni singolari si hanno nelle calotte polari, ove tale durata supera le $24^{h}\left(^{*}\right)$, e all'equatore, in cui si hanno per tutto l'anno condizioni equinoziali; cosi, le durate in questione dipendono, oltre che dalla posizione del Sole sull'eclittica (e quindi dall'epoca dell'anno), anche dalla latitudine del luogo.

${ }^{*}$ ) Il giorno siderale è circa $4^{\mathrm{m}}$ più breve del giorno solare.

$\left.{ }^{*}\right)$ Al limite, ai poli il Sole è visibile ininterrottamente per circa metà dell'anno e invisibile per la restante metà. 
Tempo solare vero. - Lasciando da parte la posizione del Sole sull'eclittica che costituisce una sorte di indicatore calendario, ed è quindi di minore importanza per le questioni che vogliamo qui dibattere, da quanto precede risulta che la posizione del Sole sulla volta celeste durante la giornata costituisce la "lancetta » di un orologio che segna mezzogiorno quando il

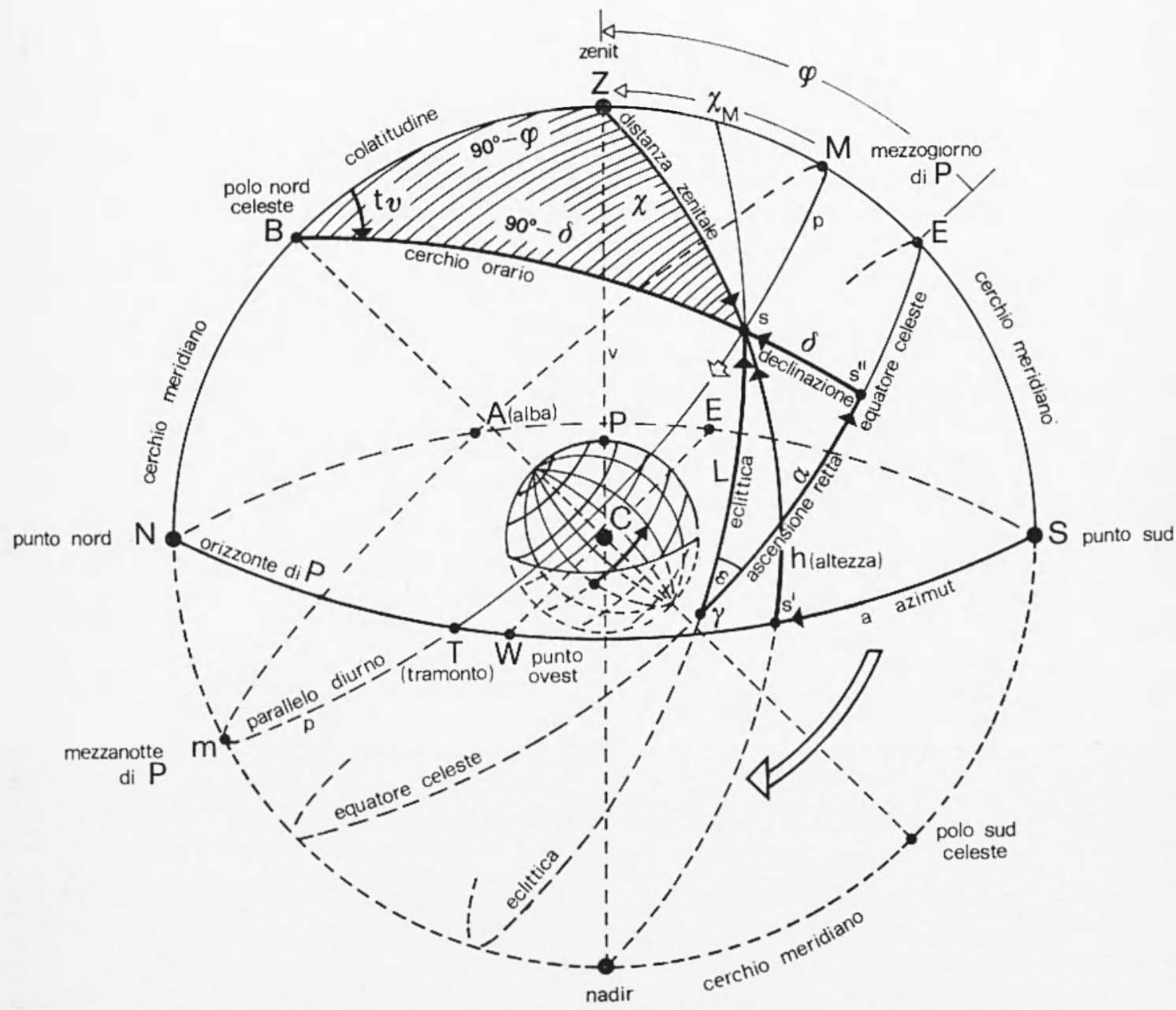

Fig. 1 - Elementi del corso diurno e annuo del Sole sulla sfera celeste. 
Sole passa al meridiano superiore del luogo, in $\mathbf{M}$ (fig. 1); in un istante generico, il tempo solare vero rispetto al mezzogiorno è misurato dall'angolo t." ("angolo orario" del Sole) che il "cerchio orario" del Sole (cerchio massimo Bs della sfera celeste passante per il polo nord celeste $\mathbf{B}$ e per la posizione $\mathbf{s}$ del Sole) forma con il cerchio meridiano del luogo, contato in verso orario da mezzogiorno a mezzanotte $\left(0 \div 180^{\circ}\right)$ e successivamente da mezzanotte a mezzogiorno $\left(180^{\circ} \div 360^{\circ}\right)$.

$E^{\prime}$ da osservare che, con ovvi adattamenti, per origine di tale tempo può essere preso (come in realtà è stato fatto nelle varie scale cronometriche) uno degli altri punti singolari del parallelo solare p, e cioè la mezzanotte o l'alba o il tramonto.

Tra questi 4 punti, quello il cui istante si coglie meglio è il mezzogiorno, in cui, come accennato, il Sole transita al meridiano superiore del luogo e è minima la sua distanza zenitale. Così, osservando l'ombra di un oggetto (un semplice bastone infisso per terra, ad es.), il mezzogiorno corrisponde all'istante in cui l'ombra ha la minima lunghezza: è questo, com'è ben noto, il principio di funzionamento delle "meridiane " (orizzontali, verticali, oblique), così chiamate proprio perché atte a segnalare il passaggio del Sole al meridiano. Della dipendenza fra la posizione dell'ombra dell'estremità di uno stilo (gnomone) su un piano di proiezione e la posizione del Sole sulla volta celeste ci si è giovati poi per costruire quelle utili varianti delle meridiane che sono gli " orologi solari ", atti a indicare il tempo solare vero.

Per verificare la circostanza del minimizzarsi della distanza zenitale del Sole a mezzogiorno e, più in generale, per presentare la relazione fra tempo solare vero e posizione del Sole sulla sfera celeste, che servirà per il seguito delle considerazioni da fare, ricorderemo che tale posizione può essere riferita a vari sistemi di coordinate sulla sfera medesima:

a) sistema altazimutale, di uso più geodetico che astronomico, ottenuto proiettando sfericamente il Sole $\mathbf{s}$ sull'orizzonte del luogo, in $\mathbf{s}^{\prime}$ (fig. 1): le coordinate sono l'azimut a (contato sull'orizzonte in verso orario a partire dal punto sud $\mathbf{S}$ ) e l'altezza $\mathbf{h}$ (contata sul cerchio verticale Zss' positivamente al disopra dell'orizzonte), pari al complemento della distanza zenitale $\%$ (l'arco Zs, contato a partire da Z);

b) sistema equatoriale, il più usato in astronomia, che si ottiene proiettando sfericamente il Sole s sull'equatore celeste, in s": le coordinate sono l'ascensione retta $x$ (contata sull'equatore in verso antiorario 
a partire dal punto vernale $\gamma$ ) e la declinazione ó (l'arco s"s di cerchio orario, contato positivamente dall'equatore al polo nord celeste $\mathbf{B}$ );

c) sistema eclitticale, usato specialmente dagli astronomi antichi, che ha per cerchio base l'eclittica e in cui unica coordinata per il Sole è la longitudine celeste $\mathbf{L}$ (contata in verso antiorario sull'eclittica a partire da punto vernale $\gamma$ ). S'è usato e s'usa tuttora misurare le ascensioni rette, le longitudini e gli angoli orari in ore, anziché in gradi sessagesimali, secondo le relazioni di equivalenza:

$$
1^{\mathrm{h}}=15^{\circ} ; 1^{\circ}=4^{\mathrm{m}} \text {. }
$$

Ciò posto, applicando un noto teorema di trigonometria sferica al triangolo BZs (il cosiddetto "triangolo fondamentale" per il Sole: vedi fig. 1) si ha la seguente relazione, in cui compare anche la latitudine geografica $\varphi$ del luogo:

$\cos \chi=\sin \mathbf{h}=\sin \varphi \sin \delta+\cos \varphi \cos \delta \cos \mathbf{t} \gamma$.

Da questa fondamentale relazione segue che l'angolo orario del Sole, e cioè il tempo solare vero, può essere dcterminato semplicemente misurando l'altezza $\mathbf{h}$ del Sole sull'orizzonte, note che siano la latitudine $\varphi$ del luogo e la declinazione $\delta$ del Sole. Ricordiamo che i valori di $\tilde{o}$ vanno dal valore zero, agli equinozi (fig. 1), fino a un valore massimo, raggiunto ai solstizi, che è pari in valore assoluto all'obliquità dell'eclittica, cioè pari a circa 23027'. Per la misurazione di $\mathbf{h}$, in passato si icorreva a un astrolabio o a un quadrante; modernamente, si usa un sestante.

Una situazione particolare si ha a mezzogiomo solare vero, cioè quando $t v=0$; dalla [1] segue ( $v$. anche fig. 1):

$$
90^{\circ}-\mathbf{h}=\chi_{\mathrm{M}}=\varphi-\delta
$$

Dunque, la distanza zenitale del Sole è effettivamente minima, nel corso della giornata, a mezzodì, quando il Sole culmina superiormente.

Un'altra situazione particolare si ha all'alba e al tramonto del Sole. Precisamente, ponendo nella [1] $\mathbf{h}=0$ (o, equivalentemente, $\chi=90^{\circ}$ ) si ha:

$$
\left(\mathbf{t}_{v}\right)_{0}= \pm \arccos (-\tan \varphi \tan \delta),
$$


relazione che dà l'angolo orario $\left(t_{1}\right)_{0}$ del Sole all'alba (segno - ) e al tramonto (segno + ). Com'è naturale, questi due istanti sono sempre equidistanti dal mezzogiorno solare in qualunque epoca dell'anno, talché per la durata dell'arco diurno $\mathbf{d}_{\mathrm{g}}$ e per la durata dell'Arco notturno $\mathbf{d}_{\mathrm{n}}$ (in ore) si hanno le relazioni seguenti:

$$
\begin{aligned}
& \mathbf{d}_{\mathrm{g}}=2 !\left(\mathbf{t}_{\mathrm{v}}\right)_{\mathrm{o}} ! \\
& \mathbf{d}_{n}=24^{\mathrm{h}}-2\left|\left(\mathbf{t}_{\mathrm{v}}\right)_{\mathrm{o}}\right|
\end{aligned}
$$

Poiché a causa della rifrazione atmosferica il Sole è ancora visibile quando in realtà è già circa $36^{\prime}$ al disotto dell'orizzonte, un valore più esatto per $\left(\mathbf{t}_{\mathrm{v}}\right)_{\mathrm{o}}$ si ottiene dalla [1] facendo in questa $\mathbf{h}=-0,6^{\circ}\left(\chi=90,6^{\circ}\right)$.

La luce solare diffusa dall'atmosfera è ancora visibile per tutto un periodo, il crepuscolo, seguente il tramonto ("crepuscolo serale ") e antecedente l'alba ("crepuscolo mattutino"); si distinguono sia all'alba sia al tramonto un "crepuscolo civile " e un "crepuscolo nautico ", corrispondenti a una depressione del Sole di $6^{\circ}$ e di $12^{\circ}$, rispettivamente. Per alcune delle scale cronometriche di cui parleremo più avanti ha importanza l'istante finale del crepuscolo serale (in cui cioè è completa l'oscurità notturno), cui corrsponde per il Sole l'angolo orario ( $\mathbf{t}_{v c}$ dato, con accettabile approssimazione, dalla relazione seguente:

$$
\left(t_{v}\right)_{0} \approx \pm \arccos \left(-\frac{\sin \varphi \sin \delta+0,104}{\cos \varphi \cos \delta}\right)
$$

La durata del crepuscolo civile è, a media latitudine, di circa $30^{\mathrm{m}}$; cresce con la latitudine e varia di pochissimi minuti durante l'anno, con massimi ai solstizi e minimi agli equinozi.

Appare chiaro che il tempo solare è un tempo "locale »: quando il Sole culmina, ciò̀ è mezzogiorno, in un determinato luogo, in altri luoghi non è in genere mezzogiorno, a meno che, naturalmente, essi non si trovino lungo il meridiano geografico del primo. 
Poiché dunque il tempo solare varia con la longitudine, ogni qualvolta si voglia valutare la contemporaneità di eventi accaduti in luoghi diversi, occorre riportare il tempo solare vero $\left(t_{v}\right)_{A}$ di ogni generico luogo $A$, di longitudine $\lambda_{A}$. a quello, $\left(t_{v}\right)_{o}$, di un luogo di riferimento $O$, di longitudine $\lambda_{o}$, mediante la relazione seguente:

$$
\left(t_{v}\right)_{o}=\left(t_{v}\right)_{\lambda}+\left(\lambda_{0}-\lambda_{\lambda}\right),
$$

dove, come accennato prima, le longitudini vanno espresse in unità di tempo.

Tempo (solare) medio locale. - Poiché la Terra descrive la sua orbita annua intorno al Sole con velocità areale costante, e quindi con velocità angolare dipendente dalla distanza TerraSole, variabile durante l'anno ne segue che il Sole percorre sulla volta celeste l'eclittica con una velocità che varia durante l'anno; a questo s'aggiunge il fatto che durante la giornata il sole percorre un parallelo della sfera celeste, rispetto al quale l'eclittica è inclinata di circa $23^{\circ} 27^{\prime}$.

L'insieme di questi due fatti fa sì che la durata del giorno solare vero viene a variare nel corso dell'anno. Poiché ben si comprendono le difficoltà pratiche che seguono a tale circostanza, si è sentita la necessità di regolarizzare il moto del Sole sulla volta celeste al fine di regolarizzare la durata del giorno.

A tal fine s'immagina di sostituire al Sole vero un astro ideale, il "Sole equatoriale medio", che percorra con velocità costante l'equatore celeste (e non l'eclittica) e che passi al punto vernale negli stessi istanti in cui vi passa il Sole vero. Cosi, la posizione del Sole medio sulla volta celeste, lungo l'equatore, costituisce la "lancetta" di un orologio ad andamento uniforme, sul quale possono essere regolati orologi ad andamento parimenti uniforme, quali gli ordinari orologi "meccanici », con oscillatore 
a pendolo o a bilanciere, gli orologi " piezoelettrici», con oscillatore elettrico a cristallo, gli orologi " atomici", con oscillatore molecolare.

L'angolo orario del Sole medio, $t_{m}$, è il tempo medio, e risulta legato al tempo solare vero $t_{v}$ dalla relazione seguente:

$$
t_{m}=t_{v}-E,
$$

dove $E$ (equazione del tempo) è l'angolo orario del Sole (vero) a mezzogiorno medio, cioè quando il Sole medio culmina superiormente $\left(t_{m}=0\right)$. I valori di $E$, conosciuti con grande precisione e riportati dagli Annuari astronomici e dalle Effemeridi nautiche, variano in maniera complessa durante l'anno, raggiungendo due massimi e due minimi che comunque non superano, in valore assoluto, $17^{\mathrm{m}}$ (fig. 2 ).

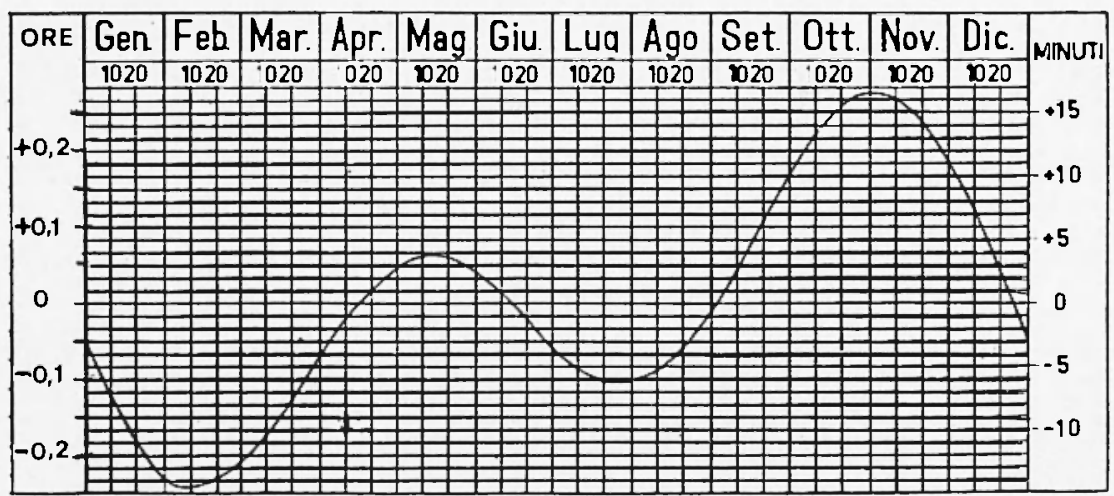

FIG. 2

Nel corso dell'anno la durata del giorno medio, cioè dell'intervallo di tempo fra due successive culminazioni superiori del Sole medio, è costante, per definizione; la sua $24 \mathrm{ma}$ parte è l'ora (solare media), con le frazioni minuto $\left(1^{\mathrm{m}}=1 / 60^{\mathrm{h}}\right)$ e secondo $\left(1^{\mathrm{s}}=1 / 60^{\mathrm{m}}=1 / 3600^{\mathrm{h}}\right)$. La durata del giorno vero invece varia 
leggermente e in maniera complessa: gli scarti massimi rispetto alle $24^{\mathrm{h}}$ di tempo medio si hanno a dicembre $\left(\approx+31^{\mathrm{s}}\right)$, aprile $\left(\approx-18^{\mathrm{s}}\right)$, giugno $\left(\approx+13^{\mathrm{s}}\right)$, settembre $\left(\approx-22^{\mathrm{s}}\right)$.

Naturalmente, il tempo medio è anch'esso un tempo locale; per riportarsi dal tempo di un luogo generico $A$ a quello di un luogo $O$ vale una relazione analoga alla [7], e cioè:

$$
\left(t_{m}\right)_{O}=\left(t_{m}\right)_{A}+\left(\lambda_{O}-\lambda_{A}\right)
$$

essendo $\lambda_{0}, \lambda_{A}$ le longitudini (espresso in ore) dei luoghi.

Tempo civile. - Per tutti gli usi civili viene adottato il cosiddetto giorno civile, che ha durata uguale a quella del giorno medio ma, a differenza di questo, viene contato da una mezzanotte all'altra, talché le misure in tempo medio vanno aumentate di $12^{\mathrm{h}}$ per avere il corrispondente tempo civile $t_{c}$.

Ricordando quanto detto in precedenza, valgono dunque le relazioni:

$$
\begin{aligned}
& t_{c}=t_{m}+12^{\mathrm{h}} \\
& t_{c}=\left(t_{v}-E\right)+12^{\mathrm{h}} .
\end{aligned}
$$

La data del giorno. - Usando il tempo civile, la data cambia, naturalmente alla mezzanotte, che è l'istante $24^{\mathrm{h}}$ per il giorno che finisce e l'istante $\mathrm{O}^{\mathrm{h}}$ per quello che inizia. Orbene, ogni qualvolta si effettua un cambiamento di tempo, passando da quello di un luogo a quello di un altro luogo con longitudine diversa, può capitare, per istanti intorno alla mezzanotte, che venga coinvolta la data.

Quest'avvertenza va tenuta presente in tutti i cambiamenti di tempo medio effettuati secondo le [9], [10], e in particolare in quelli molto frequenti in geofisica, dal tempo medio locale ai cosiddetti tempi "del fuso orario " e "universale ", e viceversa. 
Tempo del fuso orario. - Considerando i 24 meridiani terrestri di longitudine $\mathrm{O}^{\circ}$ (meridiano di Greenwich), $15^{\circ}, 30^{\circ}, \ldots$, dalle [9], [10] risulta che il tempo civile varia esattamente di un'ora passando dall'uno all'altro di tali meridiani. Secondo una proposta fatta sul finire dell'Ottocento $\left({ }^{*}\right)$, volta a rendere unica la scala delle misure orarie nell'ambito di uno stesso Stato, a tutti i luoghi situati in uno dei 24 fusi sferici centrati sui meridiani anzidetti viene attribuito il tempo civile del meridiano centrale, detto tempo del fuso orario (in particolare tempo del fuso zero per i luoghi compresi entro $t 7,5^{\circ}$ long. dal meridiano di Greenwich; tempo del fuso $1^{h}$, per quelli entro $t$ 7,5 dal meridiano $15^{\circ} \mathrm{E}$; ecc.: fig. 3 ).

Tale tempo è anche det to tempo legale $\left({ }^{* *}\right)$ perché adot tato dai singoli stati con propri provvedimenti di legge nei quali si precisavano anche eventuali adattamenti nel caso che il territorio nazionale non fosse interamente compreso in un fuso orario: così, il provvedimento con cui fu adottato tale tempo in Italia (R.D. 10 agosto 1893 , n. 490) precisava che a partire dal $1^{\circ}$ novembre 1893 per tutto il territorio nazionale valesse il tempo del fuso orario- $\mathrm{I}^{\mathrm{h}}\left(15^{\circ} \mathrm{E}\right)$, detto anche, all'inizio, tempo medio dell'Europa centrale (T.M.E.C.), e questo anche se geograficamente inesatto, giacché alcune zone della parte occidentale del Piemonte dovrebbero appartenere al fuso zero, avendo longitudine minore di $7,5^{\circ}$.

Per la conversione dei tempi locali, di cui si è parlato, in " tempo del fuso orario ", $t_{\text {, }}$ valgono le seguenti relazioni:

$$
\begin{array}{ll}
\text { per il tempo medio } & t_{f}=\left(t_{m}\right)_{A}+12^{\mathrm{h}}+\left(\lambda_{I}-\lambda_{A}\right), \\
\text { per il tempo vero } & t_{l}=\left[\left(t_{v}\right)_{A}-E\right]+12^{\mathrm{h}}+\left(\lambda_{I}-\lambda_{A}\right),
\end{array}
$$

${ }^{*}$ ) Nel 1878, dal canadese Sandford Fleming; l'idea era stata già avanzata, nel 1859, da Q. Filopanti.

$\left.{ }^{* \star}\right)$ Da non confondere con il "tempo legale " legato alla cosiddetta " ora legale estiva »: v. oltre, nota in calce a pag. 165. 


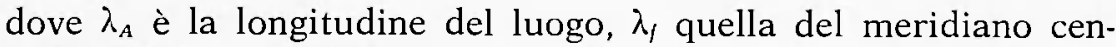
trale del fuso cui il luogo appartiene entrambe valutate in ore, ed $E$ è l'equazione del tempo.

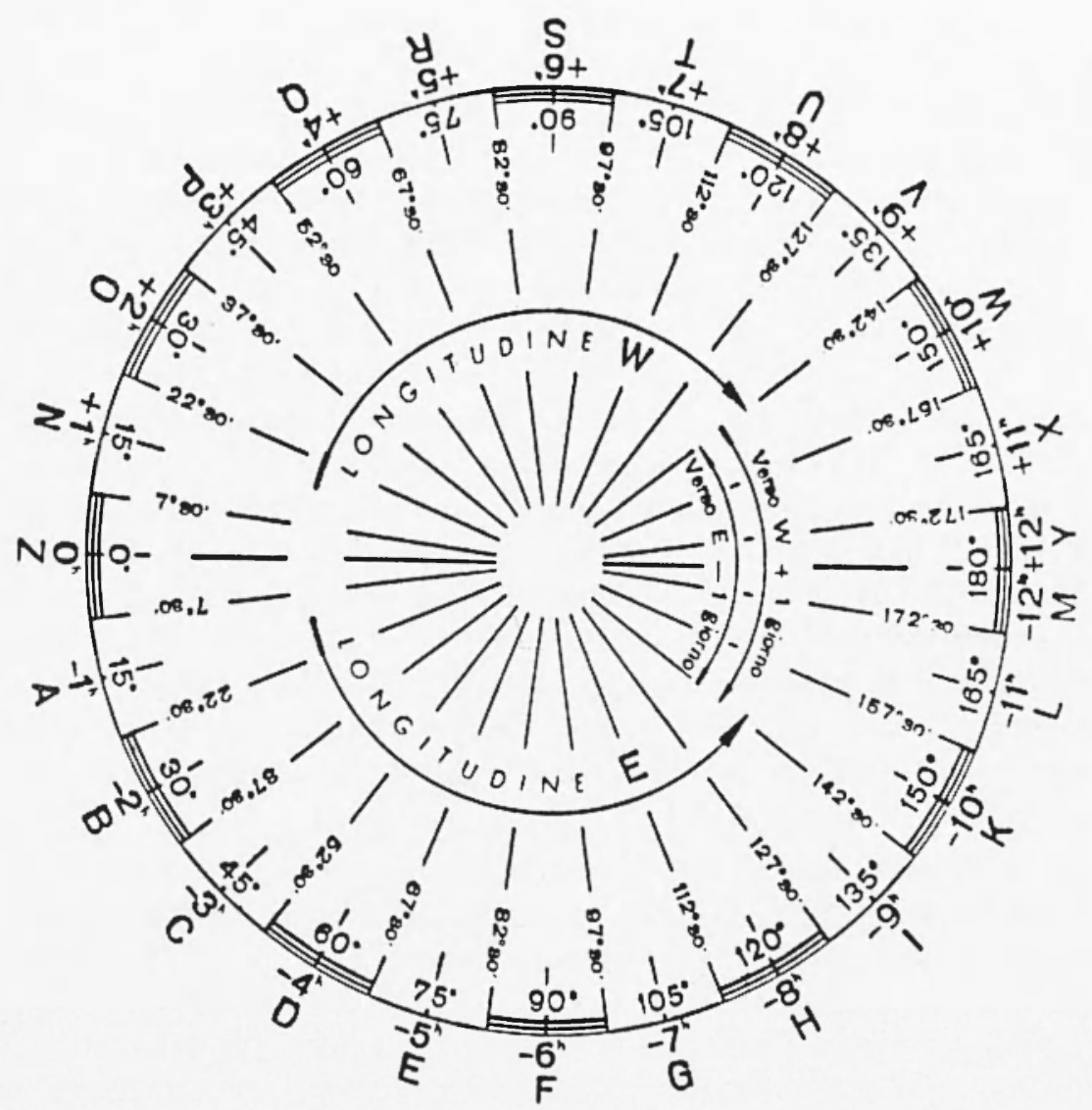

Fic. 3 - Schema dei fusi orari (da Effemeridi nautiche, Istituto Idrografiche della Marina)

Tempo universale. - Essendo utile, per la valutazione della relatività temporale di eventi svolgentisi in vari luoghi della Terra, poter riferire gli eventi medesimi a una scala di tempo valida 
per tutto il pianeta, si è assunto come tempo universale $\left({ }^{*}\right)$ il tempo civile del meridiano di Greenwich. Com'è ben noto, il tempo universale domina nelle sinossi geofisiche.

In sintesi, per la conversione in tempo universale dei vari tempi fin qui nominati, si hanno le seguenti formule

$$
\begin{array}{ll}
\text { per il tempo del fuso } & \mathrm{TU}=t_{f}-\lambda_{f} \\
\text { per il tempo medio locale } & \mathrm{TU}=\left(t_{m}\right)_{A}+12^{\mathrm{h}}-\lambda_{A} \\
\text { per il tempo vero } & \mathrm{TU}=\left[\left(t_{v}\right)_{A}-E\right]+12^{\mathrm{h}}-\lambda_{A}
\end{array}
$$

Irregolarità della rotazione terrestre. Tempo delle effemeridi e tempo atomico internazionale. - Le scale di tempo fin qui ricordate, compresa quella del tempo universale $\mathrm{TU}$, sono basate tutte sulla rotazione terrestre, sia pure regolarizzata con la sostituzione del Sole medio al Sole vero. Quando agli orologi meccanici fecero seguito orologi piezoelettrici $\left({ }^{* *}\right)$, molto più regolari, e orologi cosiddetti atomici $(* * *)$, ancora più regolari, si potè notare che tale rotazione presenta in realtà alcune irregolarità residue minori.

Tra esse, hanno rilevanza una variazione a lunghissimo periodo della durata del giorno medio, dell'ordine (attualmente) di circa $+1 \mathrm{~ms} / \mathrm{secolo}$, dovuta con tutta probabilità alla perdita di energia per attrito nei moti di marea, e una variazione stagionale di detta durata, dell'ordine di qualche $\mathrm{ms}$ fra estate e inverno dovuta, secondo quanto si presume, alla circolazione

(*) Indicato con varie sigle nelle varie lingue: TU (ital. e franc.) UT (ingl:: Universal Time), WZ (ted.: Weltzeit); anche, GMT, dall'ingl. Greenwich $M$ ean $T$ ime.

${ }^{* *}$ Entrati nell'uso intorno al 1935.

$\left(^{* * *}\right)$ Il prmo orologio del genere, oscilatore molecolare ad ammoniaca entrò in funzione nel 1948, presso i laboratori del National Bureau of Standards, negli U.S.A. 
atmosferica e forse anche alle maree. Piccole variazioni si hanno anche nell'assetto dell'asse della rotazione, con conseguenti piccole, irregolari e lente "migrazioni » dei poli $\left({ }^{*}\right)$, e variazioni delle coordinate terrestri e celesti.

Per eliminare l'effetto di tali irregolarità nello scorrere del tempo medio di anno in anno, nel $1954 \mathrm{fu}$ proposto, in seno alle associazioni internazionali astronomiche e metrologiche, di riferirsi a un anno tropico campione. Come tale fu scelto quello che iniziò alle ore zero (mezzanotte) del $1^{\circ}$ gennaio 1900 , per il quale si possedevano accuratissime effemeridi del Sole vero e del Sole medio. Nell'ottobre 1956 il Comitato internazionale dei pesi e delle misure decise di sostituire al tempo valutato sul giorno medio dell'anno in corso,, quello riferito alle effemeridi del Sole medio per l'anno tropico $1900\left({ }^{* *}\right)$, per tal motivo detto tempo delle effemeridi. Da questo tempo sono stati dedotti due "tempi universali »: uno, indicato con la sigla TU1 (o UT1), il cui scorrere è depurato dalle irregolarità associate alla migrazione dei poli terrestri; l'altro, indicato con la sigla TU2 (o UT2), depurato anche delle irregolarità associate alle variazioni stagionali della rotazione terrestre; si decise poi di indicare con la sigla TUO (o UTO) il precedente tempo universale "non corretto ».

L'uniformità di queste ultime scale di tempo è dell'ordine di $10^{-y}$. ben maggiore quindi di quella del tempo TUO, che è dell'ordine di $10^{-7}$, ma nettamente inferiore rispetto a quella del tempo indicato da orologi atomici, che è dell'ordine di $10^{-13}$. Per tale motivo e anche per la necessità di disporre di campioni di tempo riproducibili, la XIII Conferenza internazionale dei pesi e delle misure stabilì nel 1967 di abbandonare la definizione dell'unità di misura del tempo basata sulla rotazione terrestre e di riferire lo scorrere del tempo alle indicazioni di un orologio atomico del tipo a Cesio, definendo il «secondo» in

$\left(^{*}\right)$ Entro un raggio di una quindicina di $\mathrm{m}$.

$\left.{ }^{* *}\right)$ Il secondo è l'intervallo di tempo in cui sono compresi 9.192.631.770,0 periodi della radiazione elettromagnetica monocromatica che s'accompagna alla transizione fra i livelli di struttura iperfina dello stato fondamentale ${ }^{2} S_{1 / 2}$ del ${ }_{35}^{135} \mathrm{Cs}$ con $F=4, m_{i}=0$ e $F=3, m_{i}=0$. 
relazione alla frequenza della radiazione emessa in una ben definita transizione energetica degli atomi ${ }_{53}^{135} \mathrm{Cs}\left({ }^{*}\right)$. Si ebbe cosi il tempo atomico internazionale, indicato con la sigla TAI (o anche IAT, dall'inglese International Atomic Time), di cui si assunse come istante iniziale l'istante zero (di tempo TU2) del $1^{\circ}$ gennaio 1958.

Tempo universale coordinato. - Se è vero che il tempo atomico è assai più uniforme e riproducibile di quello solare medio - e questa qualità è fondamentale per le necessità della fisica e della tecnica - resta il fatto che per le necessità della vita civile - e anche in molte questioni riguardanti le scienze della Terra fattori determinanti restano l'altezza del Sole sull'orizzonte, l'alternarsi del giorno e della notte, il susseguirsi delle stagioni, cose che sono legate tutte al tempo solare vero e, per esso, al tempo medio. Tale fatto non è irrilevante se si pensa che 1'« orologio " Terra (sia pure regolarizzato al massimo con il ricorso ai tempi TU1, TU2) attualmente ritarda di quasi un secondo all'anno sugli orologi atomici (che ormai vanno considerati esatti. per definizione). Quando, all'istante zero $1^{\circ}$ gennaio 1972, il tempo atomico fu introdotto a tutti gli effetti nella vita civile, il tempo universale ritardava su di esso di ben 10 secondi, accumulati nel breve periodo di 14 anni (dal 1958 al 1972).

A questa situazione s'è messo rimedio mediante il cosiddetto tempo universale coordinato, indicato con la sigla TUC (o. all'inglese, UTC), che è il tempo TAI, fornito dunque da orologi atomici, il quale subisce però di quando in quando, e precisamente prima dell'istante iniziale di ogni $1^{\circ}$ gennaio e, se occorre, anche del successivo $1^{\circ}$ luglio (fig. 4), correzioni in ragione di un numero intero (di norma, uno) di " secondi intercalari " per riportarlo al vecchio tempo $\mathrm{TU}$, in modo che lo scarto da questo ultimo non superi 0,7 secondi.

Poiché dal $1^{\circ}$ gennaio 1972 i radiosegnali orari (sui quali sono regolati tutti gli orologi del mondo) sono emessi in tempo

(*) L'unità di misura del tempo, il secondo, fu definito come la frazione 1/31.556.925.974.7 dell'anno tropico 1900. 
TUC, l'attuale scala di tempo (sia quella del fuso orario sia quella del tempo universale) è quindi una scala uniforme che subisce periodiche discontinuità, delle quali ovviamente va tenuto conto.

Nell'Appendice A sono riportate le inserzioni di secondi intercalari fatte sino al momento in cui il presente lavoro è stato consegnato alla stampa (vedi pagina 199).

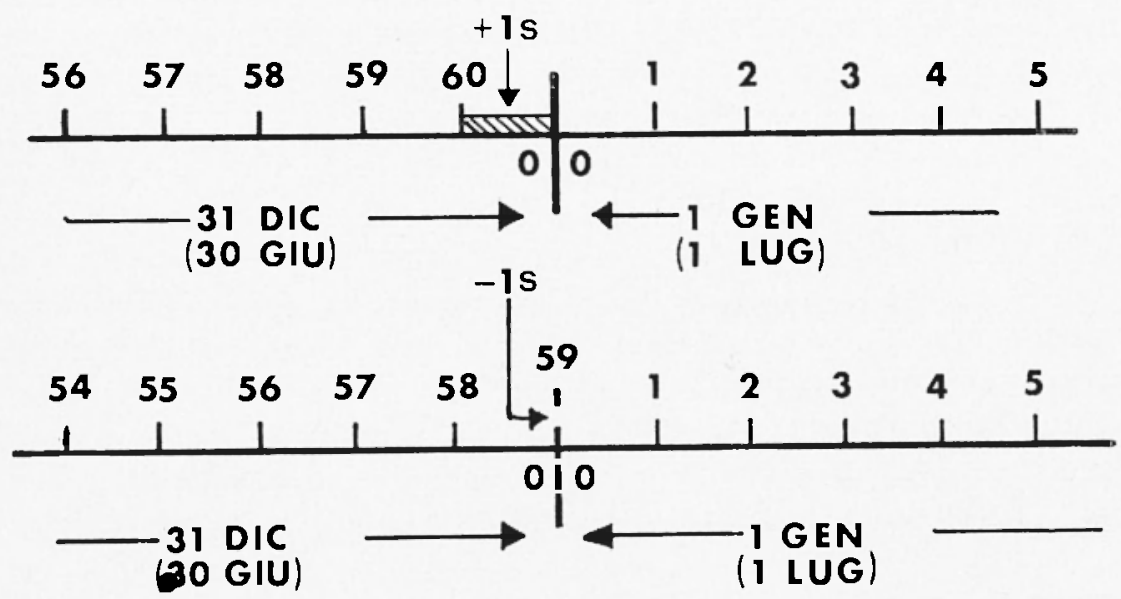

Fig. 4 - Schema dell'inserzione dei secondi intercalari del tempo TUC. Nella scala dei secondi, il numero d'ordine indica la fine di ogni secondo.

Tempo siderale. - E' il tempo fondamentale degli astronomi, definito come l'angolo orario del punto vernale.

In tutti gli osservatori astronomici v'è sempre almeno un orologio " a tempo siderale "; così, può capitare di imbattersi in descrizioni di fenomeni geofisici riferite a tale tempo. Per questo motivo riteniamo utile accennare anche alla conversione di esso in tempo civile.

Poiché gli Annuari astronomici e le Effemeridi nautiche danno il tempo siderale $t_{s m}$ alla mezzanotte media di Greenwich (e quindi alla mezzanotte media locale di un sito qualsiasi) per 
avere il tempo civile locale $t_{c}$ (e da esso poi il tempo del fuso $t_{f}$ e quello universale TU) basta convertire in intervallo di tempo medio l'intervallo di tempo siderale trascorso a partire dalla mezzanotte media precedente, tenendo conto del fatto che un giorno medio supera di $3^{\mathrm{m}} 56,56^{\mathrm{s}}$ un giorno siderale $\left({ }^{*}\right)$, a conti fatti, all'istante $t_{s}$ di tempo siderale corrispondono, nelle varie scale di tempo medio, gli istanti dati dalle seguenti relazioni:

per il tempo civile locale

$$
t_{c}=0,997.270\left(t_{s}-t_{s m}+k 24^{\mathrm{h}}\right)
$$

per il tempo del fuso

$$
t_{l}=0,997.270\left(t_{s}-t_{s m}+k 24^{\mathrm{h}}\right)+\left(\lambda_{l}-\lambda_{A}\right)
$$

per il tempo universale

$$
\mathrm{TU}=0,997.270\left(t_{s}-t_{s m}+k 24^{h}\right)-\lambda_{A}
$$

dove $k=0$ se $t_{s}>t_{s m}, k=1$ se $t_{s}<t_{s m}$, e inoltre sia i tempi siderali sia le longitudini ( $\lambda_{f}$ del fuso e $\lambda_{A}$ del luogo) sono espressi in ore.

(*) In un anno tropico, che contiene 365,2422 giorni medi, sono contenuti 366,2422 giorni siderali, talché il rapporto fra misure di uno stesso intervallo di tempo in unità di tempo medio e unità di tempo siderale è pari a $365,2422 / 366,2422 \approx 0,997.270$. 


\section{PARTE SECONDA}

\section{Rassegna storica delle scale cronometriche succedutesi in Italia.}

Alle considerazioni di carattere meramente tecnico fin qui esposte faremo ora seguire alcune considerazioni, questa volta di carattere principalmente storico, sui vari modi che nel nostro paese sono stati via via seguiti per indicare il trascorrere del giorno.

Periodo romano. - I Romani, come tutti i popoli dell'antichità, distinguevano nettamente il corso diurno del Sole da quello notturno: essi parlavano di dies naturalis per indicare l'intervallo di tempo, compreso fra l'alba e il tramonto, in cui il Sole è sopra l'orizzonte, e di dies civilis (il vuxî́nuepov greco), che era poi il giorno per antonomasia, per indicare l'insieme del dies naturalis e della nox ad esso associata, e precisamente il periodo da una mezzanotte alla successiva $\left({ }^{*}\right)$.

Seguendo poi un uso praticato dai Greci (introdotto dai Babilonesi e diffuso dai Fenici in tutto il Mediterraneo), dividevano sia il corso diurno sia la notte in quattro parti, ciascuna delle quali suddivisa in tre ore. Le quattro parti del corso diurno del Sole erano chiuse, rispettivamente, dalle ore tertia, sexta, nona

${ }^{(*)}$ Un'eccellente sintesi si ha nel seguente passo della monumentale opera di erudizione Etymologiarum, di Isidoro di Siviglia (560-636):

DE DIEBUS. Dies est presentia solis, sive sol supra terras, sicut nox sol sub terris... Dies legitimus viginti quattuor horarum, usque dum dies et nox spatia sui cursus ab oriente usque ad alium orientalem solem caeli volubilitate concludat. Abusive autem dies unus est spatium ab oriente sole usque ad occidentem. Sunt autem diei spatia duo, interdianum atque nocturnum; et est dies quidem horarum viginti quattuor, spatium autem horarum duodecim... Dies secundum Aegyptios inchoat $a b$ ocasu solis: secundum Persas ab ortu solis: secundum Athenienses $a$ sexta hora diei; secundum Romanos a media nocte. (lib. $\mathrm{V}, \mathrm{XXX}$ ). 
e duodecima (quest'ultima coincidente con il tramonto, Solis occasus) corrispondenti, agli equinozi, alle nostre ore 9, 12, 15, 18; analogamente per le parti della notte, dette vigiliae. Usavano poi denominazioni particolari, con definito significato cronometrico, per momenti o periodi speciali che scandivano il giorno e la notte: cosi, chiamavano mane la prima parte dell'arco diurno, meridies il mezzogiorno (allo spirare della hora sexta), suprema l'ultima parte del giorno, verso i tramonto. Una corrispondenza approssimativa tra queste indicazioni orarie romane sia diurne sia notturne $\left({ }^{*}\right)$ e le nostre ore attuali è indicata nella fig. 5, relativamente a periodi intorno agli equinozi.

Quest'ultima precisazione è necessaria in quanto, con la costante ripartizione dell'arco diurno e di quello notturno in 12 ore, venivano ad aversi ore diurne e notturne di ineguale durata durante l'anno (horae inequales o horae temporae): più lunghe le diurne e più corte le notturne nei mesi estivi, e il contrario nei mesi invernali. Soltanto agli equinozi quelle ore erano uguali fra loro, e coincidenti con quelle che s'usano oggidì.

I Romani ereditarono dai Greci e dagli Egizi anche le loro nozioni astronomiche, che erano più vaste e accurate di quel che si sarebbe portati a pensare: questi popoli possedevano, per es., le nozioni di tempo siderale, tempo solare vero, tempo medio.

Per la determinazione dell'ora del giorno i Romani furono per parecchio tempo sprovvisti di strumenti sufficientemente precisi, sino al momento in cui vennero a contatto con le evolute colonie greche del centrosud. Il primo orologio solare fu installato a Roma, nel Foro, presso il

(*) Noctis partes septem sunt, id est vesper, crepusculum, conticinium, intempestum, gallicinium, matutinum, diluculum. Vesperum $a b$ stella occidentali (Venere) vocatum, quae solem occiduum sequitur et tenebras sequentes praecedit... Crepusculum est dubia lux... hoc est inter lucem et tenebras. Conticinium est quando omnes silent... Intempestum est medium et inactuosum noctis tempus, quando agi nihil potest... Gallicinium propter gallos lucis praenuntios dictum. Matutinum est inter abscessum tenebrarum et aurorae adventum.. Diluculum quasi iam incipiens parva diei lux. Haec et aurora, quae solem praecedit. Est autem aurora diei clarescentis exordium et primus splendor aeris... Isidoro di Siviglia, loc. cit., XXXI. 
tempio di Quirino, da L. Papirio Cursore nel 263 a.C. ma non era preciso, dal momento che era stato preso ai Sanniti e quindi non era stato costruito per la latitudine di Roma.

Prima di questo orologio, i Romani avevano a disposizione soltanto gli annunci di un littore che, nel Foro, scandiva i quarti del giorno; per i frequentatori del Foro, il mezzogiorno era indicato dall'apparire del Sole fra i Rostri e la Grecostasi. Nel 263 a.C. M.M. Valerio Messalla installò a fianco del precedente un altro orologio solare, trasportato da Catania e quindi ancora meno preciso. Soltanto nel 56 a.C. il patrigno di Augusto, Q. Marcio Filippo, fece costruire un orologio solare per la latitudine di Roma. Dopo di allora, i Romani progredirono rapidamente, dotandosi di tutti gli strumenti allora disponibili per determinare l'ora meridiane (per cogliere l'istante del mezzogiorno vero), astrolabi (per misurare l'altezza di astri sull'orizzonte e quindi, mediante la [1], angoli orari) e orologi solari, sia fissi sia portatili.

Per "conservare» il tempo fra un'osservazione astronomica e l'altra si ricorreva a clessidre, di vari ingegnosi tipi (a stillazione; a immersione; ignee, ciò̀ a combustione di ceri, ecc.) e ad orologi, sia "idraulici " (in cui lo scorrere del tempo veniva riferito al fluire d'una corrente d'acqua, regolarizzato con opportuni dispositivi) sia "meccanici" (basati sulla discesa di un peso, resa sensibilmente uniforme mediante ventole, volani e rotismi) $\left(^{*}\right)$. Le clessidre e gli orologi idraulici e meccanici indicavano ovviamente un tempo che scorreva uniformemente, e quindi indicavano ore di uguale durata (horae equales o equinoctiales) (**). Nell'amministrazione civile e in quella militare erano peraltro in uso anche clessidre a ore temporarie, le quali erano provviste di un meccanismo di regolazione, da aggiustare a seconda dell'epoca dell'anno in modo che la durata della hora indicata dalla clessidra corrispondesse alla dodicesima parte dell'intervallo di tempo fra l'alba e il tramonto del Sole a quell'epoca.

\section{DAL MEdIOEVo ALL'ETA' MODERNA.}

Le ore temporarie liturgiche. - Dopo la caduta dell'Impero Romano d'Occidente, la cronometria romana fu conservata, sia pure con modificazioni, dalla Chiesa cristiana. In Oriente, ma con evoluzione abbastanza diversa, fu conservata dall'Impero

${ }^{*}$ ) I primi orologi di questo genere pare che risalgano al sec. $9^{\circ}$ a.C. (L. Volta, in Enciclopedia Italiana, XXXIII, p. 478).

(**) Per es., clessidre di questo genere erano usate, già presso i Greci, per misurare l'intervallo di tempo assegnato per le arringhe dei patrocinatori nei processi. 
Bizantino e dagli Arabi, e da questi ultimi ritornò poi migliorata (soprattutto per quanto riguardava l'arte di costruire buoni strumenti) all'Occidente dopo la conquista araba dell'Italia meridionale e della penisola iberica.

La Chiesa latina mantenne, per regolare i suoi riti, le ore temporarie romane ma introdusse, sin dalle origini, un'importante novità, e cioè il computo del giorno da un tramonto del Sole al successivo, secondo l'uso religioso ebraico e, in genere, di tutti i popoli del vicino Oriente (giorno cosiddetto «mosaico » o «biblico", cui si attengono tuttora, per il culto, gli Ebrei e i mussulmani; in antico, però, i Babilonesi facevano iniziare il giorno al levar del Sole: giorno cosiddetto "babilonese»).

Lo scorrere del tempo era segnato da preghiere e riti particolari a seconda del momento della giornata: l'alba (preghiera delle «Lodi »), l'ora terza (santificata dalla discesa dello Spirito Santo sugli Apostoli), la ora sesta (la crocifissione di Gesù), l'ora nona (la morte di Gesù). Molto presto, presumibilmente per sottrarre i fedeli alle persecuzioni, alle preghiere furono interessate anche le ore notturne, cioè le vigiliae: alle ore dell' " ufficio diurno " s'aggiunsero così quelle dell'ufficio notturno » ("vespro », " compieta », "notturno ", mattutino », "lodi »: variamente spostate, queste ultime, fra due ore e un'ora prima dell'alba), le ore canoniche venendosi a configurare all'incirca come indicato nella fig. 5.

Dopo il sec. $4^{\circ}$, la libertà di culto di cui la Chiesa romana venne a godere dette luogo a un'altra importante innovazione, e cioè quella di annunciare il momento delle preghiere, come dire le ore canoniche, con il suono delle campane: consuetudine che, insieme alle ore canoniche medesime, si diffuse in tutta l'Europa, specialmente a opera dei benedettini. In tal modo la conoscenza dell'ora del giorno, prima generalmente ristretta a poche persone, veniva a essere, in un centro abitato, uniformata e diffusa nell'ambito, piuttosto vasto, del raggio di udibilità delle campane. Più tardi, circa a partire del sec. $14^{\circ}$, le campane furono azionate meccanicamente per «battere» le ore, mediante appositi 
dispositivi applicati ai grandi orologi meccanici a discesa di pesi che in numero sempre maggiore presero a essere montati sui campanili e sulle torri civiche delle città e dei borghi $\left({ }^{*}\right)$.

Ore equinoziali all'italiana. - Proprio il diffondersi di orologi meccanici, e soprattutto di quelli con soneria a campane, portò presto a togliere alle ore liturgiche il compito di regolare il corso della vita civile, per la qual cosa meglio conveniva l'uniforme trascorrere delle ore segnate da quegli orologi. Per di più, gli usi ecclesiastici erano stati più volte modificati $\left({ }^{* *}\right)$. Così, le ore cano-

${ }^{(*)}$ Notizie certe sull'esistenza di questi orologi che battevano le ore si hanno a partire dai primi anni del 1300 , pressoché contemporaneamente in tutta l'Europa (precedentemente, si hanno notizie di orologi idraulici e meccanici a pesi, qui per aquam fiunt et pondera).

Il primo orologio pubblico di cui si ha notizia certa per l'Italia fu montato a Milano nel 1336 (G. Bilfiger, Die mittelalterlicher Horem und die modernen Stunden, Stuttgart 1892, pag. 175 e sgg.); altra notizia certa riguarda un orologio pubblico con soneria a campane costruito e installato a Padova intorno al 1340 da Iacopo Dondi, capostipite di una celebre dinastia di costruttori di orologi da torre e da appartamento. Ma orologi meccanici a rotismi dovevano essere abbastanza diffusi in Italia anche prima; basti questo esplicito accenno che ad essi fa Dante nella Commedia (Paradiso, c. XXIV, 13-15):

E come cerchi in tempra d'orioli

si giran si, che 'l primo a chi pon mente

quieto pare, e l'ultimo che voli;

con riferimento alla diversa velocità delle ruote.

A titolo di curiosità, ricorderemo che nella Commedia v'è un altro riferimento a orologi, e precisamente a orologi meccanici provvisti di sveglia (Paradiso, c. X, 139-144):

Indi, come orologio che ne chiami

tin tin sonando con si dolce nota

$\left.{ }^{* *}\right)$ In particolare riportando l'inizio del giorno alla mezzanotte, secondo l'uso romano.

Al riguardo, nella Summa Theologica di S. Tommaso d'Aquino (scritta intorno al 1273) si legge (Tertia pars, q. 80, a. 8, Ad V):

Et licet principium diei secundum diversos diversimode sumatur, 


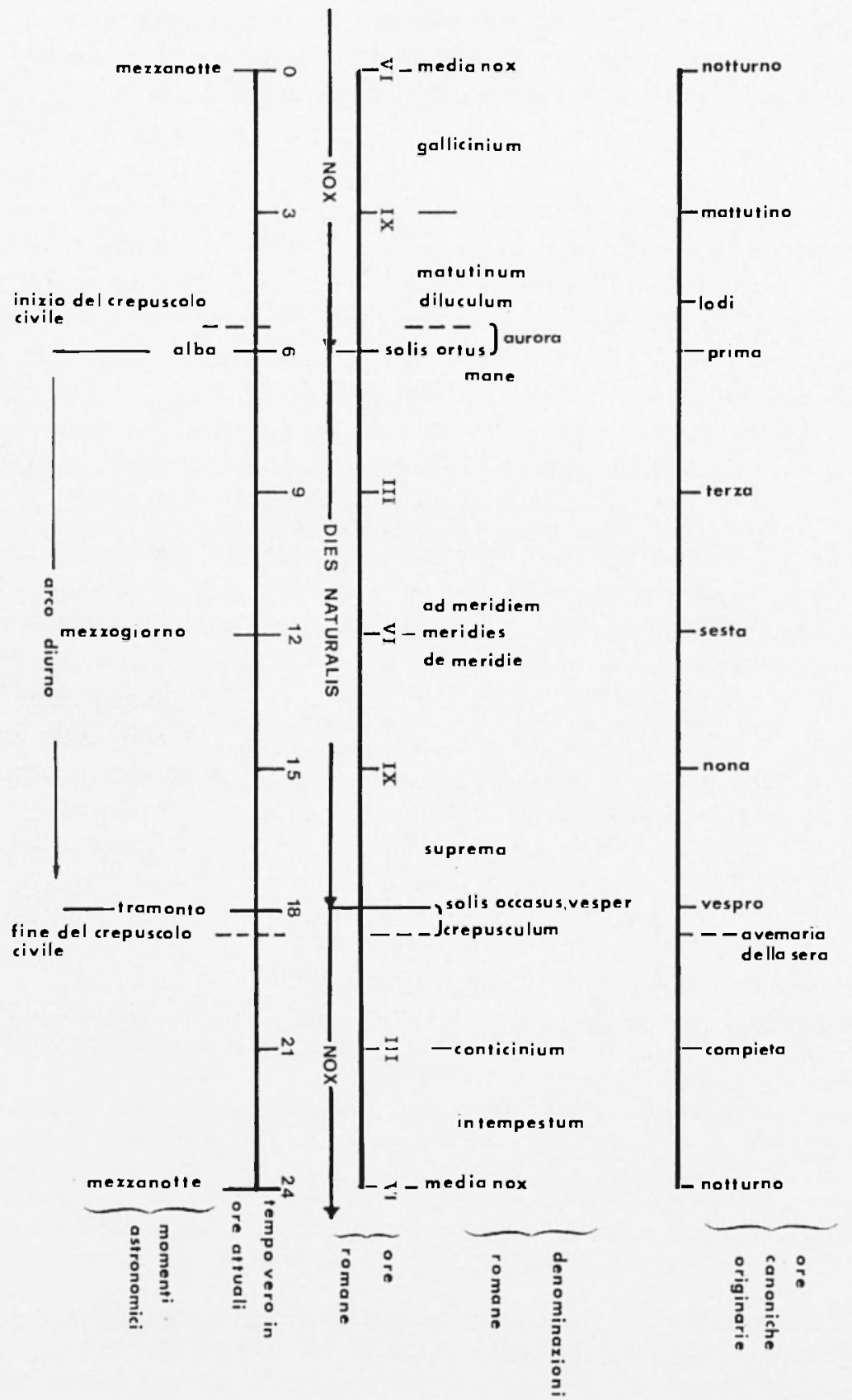

Fig. 5 - Corrispondenza, indicativa per periodi intorno agli equinozi, fra ore attuali, ore romane e ore canoniche. 
niche " finirono abbastanza presto per perdere ogni preciso e immutabile contenuto cronometrico per assumere il significato, che è quello proprio di esse oggidi, di una serie ordinata di preghiere giornaliere.

Per questo motivo, dai primi decenni del $14^{\circ} \mathrm{sec}$. circa, le cronache (come meglio vedremo nella terza parte di questo lavoro) cominciano a riportare gli avvenimenti in una scala di tempo formatasi spontaneamente per gli usi civili a partire dal primitivo computo ecclesiastico del giorno e che presto, diffusasi in tutti i paesi europei, fu detta delle ore all'italiana, o ore italiane.

Secondo questa scala il giorno era di 24 ore equinoziali. cioè uguali fra loro, contate a partire dal tramonto: anzi, come quasi subito si costumò, a partire dall' "avemaria della sera ", o "Angelus della sera", indicato - secondo una consuetudine mai interrottasi in Italia - dalle campane delle chiese e dei conventi alla fine del crepuscolo serale, vale a dire - come già precisato una mezz'ora dopo il tramonto.

In Italia questo modo di indicare le ore del giorno divenne pressoché esclusivo a partire dal 1400 circa, dominò nelle cronache per tutto il 1600 e quasi tutto il 1700, restando in ampio uso anche nei primi decenni del $1800\left(^{*}\right)$.

In tutto questo periodo prosegui però, anche se sempre più sporadicamente, l'uso cronachistico ed epigrafico di ore di notte e ore di giorno (dette allora horae antiquae), cioè delle ore

nam quidam a meridie, quidam ab occasu, quidam a media nocte, quidam $a b$ ortu solis diem incipiunt; Ecclesiam tamen, secundum Romanos, diem a media nocte incipit. Et ideo, si post mediam noctem aliquis sumpserit aliquid per modum cibi vel potus, non potest eadem die hoc sumere sacramentum (si tratta dell'Eucarestia): potest vero si ante mediam noctem.

Ancorché debba essere letto nel suo contesto, che è quello di una prescrizione sacramentale, questo passo indica che già a quell'epoca la Chiesa contava i giorni a partire dalla mezzanotte vedi anche in calce a pag. 161).

il principio e il fine del giomo è determinato secondo gli usi delle

(*) In un noto Dizionario Enciclopedico compilato da A. Bazzarini e stampato a Veneza nel 1831, alla voce GIORNO si legge (vol. III, pag. 285, inizio $2^{\circ}$ col.): 
temporarie per ciascun arco di tempo dal tramonto all'alba e dall'alba al tramonto rispettivamente: uso che ancora sopravvive, specie per le prime ore della notte, nei nostri costumi regionali.

IL COMPUTO DELLE ORE EQUINOZIALI IN EuROPA.

Può essere utile ricordare che l'accennata diffusione del computo delle ore all'Ttaliana $\mathrm{fu}$ generale in Europa per tutto il Rinascimento, dopo di che si svilupparono varie particolarità nei diversi paesi.

Cosi, per esempio, in Francia e nei paesi che subivano l'influenza francese l'uso di contare il giorno a partire dall'avemaria della sera si alternò con l'uso di contare dalla mezzanotte (hora gallicana), uso che divenne poi prevalente.

Così pure, per influsso dei paesi germanici, in cui il computo delle ore e conseguentemente il modo di indicare le ore sugli orologi era duodecimale (cioè in due serie di dodici ore), già nel 1300 accanto alle ore italiane $\left(^{*}\right)$ cominciò a comparire la divisione delle 24 ore in 12 ore antimeridiane, da mezzanot te a mezzogiorno, e 12 ore pomeridiane, da mezzogiorno a mezzanotte, divisione tuttora vivissima anche da noi.

Nei secoli $14^{\circ}$ e $15^{\circ}$, e fino circa alla metà del secolo $16^{\circ}$, usò di fare iniziare il giorno all'alba in Gran Bretagna (hora britannica) nonché in Boemia e in Ungheria (hora bohemica), secondo l'antico uso babilonese.

varie nazioni. Gli Egizi lo incominciavano a mezzanotte; $i$ Caldei $e$ i Babilonesi al levar del sole; gli Ebrei $e$ gli Ateniesi al tramonto: così pure fanno gli Italiani moderni. Gli astronomi e $i$ nautici lo contano da un mezzogiorno all'altro, e l'uso poi ordinario e comune ̀̀ d'incominciarlo alla mezzanotte e dividerlo in due parti. Tale $\grave{e}$ il giorno naturale, civile ed ecclesiastico.

E' bene osservare che "l'uso ordinario e comune" è quello della Chiesa, con 1 significato che $\mathrm{i}$ termini "ordinario " e "comune " hanno nella liturgia.

(*) Tuttora nell'arte orologiaia si chiama "quadrante allitaliana» il quadrante con 24 ore; ma poiché lo sviluppo di tale arte avvenne, come è ben noto, soprattutto nei paesi tedeschi, si impose il quadrante a 12 ore. 
Infine, nei paesi di lingua tedesca - e così in essi si costuma ancora - vigeva l'uso dell'hora incipia (che cominciò a Basilea nel 1422), anziché quello, italiano e francese, dell'hora completa: per esempio l'istante (in notazione attuale) $10^{\mathrm{h}} 30^{\mathrm{m}}$, in Italia scritta $10^{1 / 2}$ e intesa come corrispondente a mezz'ora oltre 10 ore complete, mentre nei paesi germanici veniva scritta $1 / 211$ (ma anche 11/1/2), come corrispondente a mezz'ora dopo l'inizio dell'undicesima ora.

RIFLESSI SUlLA DATA DEL GIORNO.

Le particolarità, sopra descritte, dell'istante da cui far iniziare il computo delle ore del giorno hanno evidente rilevanza per la cronologia. In particolare, per le ore italiane la data cambia all'avemaria della sera, e di ciò va tenuto conto ogni qualvolta si debba attribuire la data a eventi accaduti fra l'avemaria e la mezzanotte, che nelle cronache possono essere riportati con la data del giorno seguente (secondo i criteri attuali). Abbiamo detto "possono essere " e non "sono riportati » in quanto da cognizioni effettuate su fonti storiche, abbiamo tratto l'impressione che al riguardo non esistesse uniformità di comportamento(*).

AVVENTO DELLE ORE ANTIMERIDIANE E POMERIDIANE DI TEMPO MEDIO.

Sul finire del sec. $18^{\circ}$ l'uso di contare le ore "all'italiana " subi notevoli modificazioni a seguito degli avvenimenti politici che fra la fine di quel secolo e l'inizio del successivo agitarono il nostro paese.

(*) La stessa Chiesa se, come detto prima, fa iniziare il giorno dalla mezzanotte, ha però sempre mantenuto il criterio di considerare appartenente all'Ufficio di un Santo anche il periodo fra il tramonto e la mezzanotte precedente il giorno in cui il Santo viene festeggiato.

Comunque, questa importante questione del cambiamento della data del giorno sarà ripresa più avanti, nell'ambito dell'analisi di misure orarie riguardanti eventi sismici. 
La conquista dell'Italia da parte delle armate napoleoniche e l'inserzione a vario titolo delle regioni storiche italiane nel sistema politico-amministrativo francese (iniziatasi con la prima campagna italiana di Napoleone, 1796, assestatasi intorno al 1805 e terminata poi con la caduta di Napoleone, 1814, e le successive restaurazioni) ebbero l'effetto di introdurre in Italia il computo delle ore "alla francese" cioé come accennato in precedenza, a partire dalla mezzanotte, in ore antimeridiane e pomeridiane.

In un primo periodo queste ore (come le ore italiane che le precedettero) erano ore di tempo solare vero, in quanto gli orologi erano universalmente regolati mediante osservazioni fatte con meridiane normali cioè a tempo vero. Col passare degli anni crebbe però il numero delle persone capaci di rimettere al tempo medio un orologio meccanico controllato mediante una meridiana, cosicché le ore cominciarono ad essere espresse anche in tempo medio (ovviamente locale).

Questa riforma cronometrica, consistente nella sostituzione delle ore italiane con ore (antimeridiane e pomeridiane) di tempo medio locale $\left({ }^{*}\right)$, ebbe una ineguale progressione; genericamente parlando essa si compi dapprima nelle grandi città, poi in quelle piccole e nei paesi delle regioni settentrionali, successivamente in quelle centrali e da ultimo in quelle meridionali e insulari dove in questo periodo di confusione, l'influenza delle mode straniere fu minore e più superficiale.

\section{IL TEMPO MEDIO DI RoMA.}

A mettere un pò di ordine intervenne nel 1866, quando il nostro paese aveva raggiunto quasi completamente l'unità politica, l'adozione (R.D. 22 settembre 1866 n. 3224) nelle provincie continentali del Regno (Sicilia e Sardegna rimasero con i tempi locali di Palermo e di Catania) del tempo medio del meridiano

(*) In campo astronomico, la riforma di cui si sta parlando fu avviata nel 1816, allorché l'autorevole Annuario francese "La connaissance des temps" iniziò a servirsi del tempo medio (in luogo del tempo vero) del meridiano di Parigi. 
di Roma (contato dalla mezzanotte e cioè tempo civile) per gli usi della pubblica amministrazione e specialmente dei servizi telegrafici e ferroviari.

Era l'epoca in cui anche altrove presero a essere usate "ore nazionali »: in tutta la Francia l'ora del meridiano di Parigi, in Inghilterra l'ora del meridiano di Londra (Greenwich), in Germania quella del meridiano di Berlino, e via dicendo. L'ora veniva distribuita in tutto il territorio nazionale mediante gli orologi degli uffici telegrafici e delle stazioni ferroviarie, che erano sincronizzati fra loro mediante il telegrafo; su quegli orologi potevano poi essere regolati altri orologi pubblici e anche gli orologi dei privati cittadini.

In realtà (come avremo modo di far rilevare nella terza parte di questo lavoro) l'uso del tempo medio di Roma penetrò piut tosto lentamente nella vita quotidiana: ancora una decina d'anni dopo l'adozione ufficiale di esso, gli orologi pubblici di varie città, anche importanti, seguitavano a marciare con il tempo medio locale, talora addirittura con il tempo solare vero.

Relativamente al tempo medio di Roma, che dunque fu per un certo periodo il tempo ufficiale nelle provincie continentali del Regno, va ricordato che la città di Roma ha avuto nell'età

Il primo di essi ebbe sede in Vaticano e fu dotato, fra l'altro, di una buona meridiana (1579 nella cosiddetta Torre dei venti); prima della sua ricostituzione (1891), ebbe però scarsa rilevanza rispetto al più famoso Osservatorio del Collegio romano. Questo si può far risalire alle osservazioni astronomiche dei padri gesuiti $C$. Scheiner e altri, fatte al tempo di Galilei, nel palazzo detto appunto "Collegio romano" che la Compagnia possedeva non lontano dal Campidoglio, e come Osservatorio in senso moderno può essere datato al 1787, quando ebbe stabile sede nella torre appositamente eretta sull'angolo sud-est di quel palazzo.

Nel 1827 fu creato il terzo Osservatorio quello del Campidoglio, che, a differenza del precedente, era un Osservatorio pubblico destinato specialmente a soddisfare esigenze didattiche per l'università di Roma.

Nel 1878 anche l'Osservatorio del Collegio romano divenne un ente pubblico fondendosi con l'Ufficio Centrale di Meteorologia che ebbe sede nello stesso palazzo. Nel 1923 i due Osservatori, del Collegio romano e del Campidoglo, furono riuniti sotto l'unica denominazione di Osservatorio di Roma, la cui sede fu portata nel 1938 sul Colle di Monte Mario, dov'è ancor oggi. 
moderna e in prosieguo di tempo quattro Osservatori astronomici.

Nella tab. 1 sono riportate le coordinate geografiche di questo Osservatorio.

Nel periodo in cui ebbe corso il tempo medio di Roma, questo fu riferito al meridiano dell'Osservatorio del Collegio Romano.

TAB. 1 - COORDINATE DEGL.I OSSERVATORI ASTRONOMICI DI ROMA

\begin{tabular}{|c|c|c|}
\hline Osservatorio & lat. $\mathrm{N}$ & long. E \\
\hline Vatic. $(1579 \ldots$ 1891-1935) & $41^{\circ} 54^{\prime} 06^{\prime \prime}$ & $12^{\circ} 27^{\prime} 17^{\prime \prime}=49^{\mathrm{m}} 49.13^{\mathrm{s}}=0,830.31^{\mathrm{h}}$ \\
\hline Coll. Rom. (1787-1922) & $41^{\circ} 53^{\prime} 54^{\prime \prime}$ & $12^{\circ} 28^{\prime} 51^{\prime \prime}=49 \mathrm{~m} 55.34^{\mathrm{s}}=0,832.04^{\mathrm{h}}$ \\
\hline Campidoglio (1827-1938) & $41 \circ 53^{\prime} 33^{\prime \prime}$ & $12^{\circ} 29^{\prime} 05^{\prime \prime}=49 \mathrm{~m} 56.33^{\mathrm{s}}=0.832 .31^{\mathrm{lt}}$ \\
\hline Monte Mario dal 1938 & $41^{\circ} 55^{\prime} 25^{\prime \prime}$ & $12^{\circ} 27^{\prime} 08^{\prime \prime}=49^{\mathrm{m}} 48.53^{\mathrm{s}}=0,830.15^{\mathrm{h}}$ \\
\hline
\end{tabular}

Adozione dEL TEMPo MEdio dELl'Europa cENTRALE.

Con R.D. n. 490 in data 10 agosto 1893 dal 1 novembre $1893 \mathrm{fu}$ adottato, per il territorio del Regno d'Italia, il tempo medio dell'Europa centrale cioè, con dizione più moderna, il tempo del fuso orario $-\mathrm{l}^{\mathrm{h}}$; questo tempo venne a lungo chiamato anche tempo dell'Etria, in quanto il meridiano centrale del fuso orario di esso $\left(15^{\circ} \mathrm{E}\right)$ passa per il cratere di quel vulcano.

Dopo il 1893 l'uso ufficiale di dividere le ore in antimeridiane e pomeridiane si ridusse gradualmente fino a tornare alla vecchia consuetudine italiana e francese di indicare le ore da $\mathrm{l}^{\mathrm{h}}$ a 24h (nell'uso corrente sopravvive però, come detto, la consuetudine duodecimale).

(*) Nel 1935 la strumentazione, arricchita, fu trasportata nella Specola vaticana di Castel Gandolfo, presso Roma, inaugurata in quell'anno. 


\section{OrA LEGALE ESTIVA.}

Nell'arrivare ai giorni nostri vi sono da rilevare soltanto due importanti avvenimenti cronometrici, e cioè l'adozione dell'ora legale estiva e l'adozione del tempo universale coordinato con i relativi "secondi intercalari".

Per quanto riguarda il tempo coordinato, di esso abbiamo parlato diffusamente al termine della prima parte pag. 150 quanto all'" ora legale estiva ", si tratta di una scala di tempo civile che ritarda sull'ordinario tempo del fuso orario per un'ora esatta; essa viene adottata durante i mesi estivi (di qui la denominazione) $\left({ }^{*}\right)$, per conseguire alcuni vantaggi, soprattutto relativamente a risparmi di energia elettrica per illuminazione e altri usi civili. Fu introdotta in vari paesi europei durante la prima guerra mondiale su proposta dell'inglese W. Willet (1915).

In Italia l'ora legale estiva è stata adottata durante le due guerre mondiali e negli immediati dopoguerra, e poi, sistematicamente, a partire dal 1966, come precisato nell'appendice B.

Vale la pena di osservare che per l'Italia, in vigenza dell'ora legale estiva vi sono due ore di differenza, e non piu una soltanto, rispetto al tempo universale.

(*) Propriamente dovrebbe chiamarsi semplicemente ora legale in quanto adottata di volta in volta con appositi provvedimenti di legge; per evitare però equivoci con l'altra e più importante "ora legale" cioè l'ora del fuso orario, è preferibile la locuzione " ora legale estiva ", anche se essa riguarda un periodo più lungo dell'estate propriamente detta (in alcuni paesi, e in passato anche in Italia, essa dura o è durata per l'intero anno). 


\section{PARTE TERZA}

\section{Precisazione delle scale di TEMPo E INTERPRETAZIONE SUI TEMPI DI EVENTI SISMICI.}

Considerazioni introduttive. - Tornando a questioni propriamente cronometriche resta da dire come le misure espresse nelle scale di tempo precedentemente descritte possano interpretarsi in tempo universale quale sia la precisione attribuibile ad esse.

Per quanto concerne l'interpretazione delle misure si può fare un discorso piuttosto generale, ma per quanto concerne la stima della precisione delle misure si entra nello specifico, per cui i criteri di valutazione possono essere diversi se si riguardano le cose sotto un profilo meramente storiografico (accettando quindi un'approssimazione piut tosto larga) oppure se si cerca di trarre dalle indicazioni orarie il massimo di informazione (e

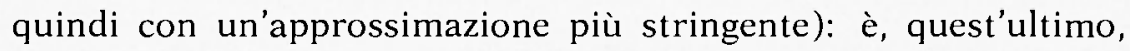
il caso di misure temporali riferentisi a eventi geofisici.

Diciamo subito che un esame poco critico in fatto di cronologia può portare autori diversi a dare informazioni di tempo differenti per uno stesso evento, con il rischio successivo che l'evento in questione venga considerato come due (o più) eventi distinti: questo rischio - cui abbiamo già accennato nella introduzione generale di questo lavoro - è particolarmente grave nella redazione di un catalogo $\operatorname{sismico}\left({ }^{*}\right)$. Per tale motivo condurremo la discussione esemplificando su dati orari di terremoti.

Dato il carattere puramente dimostrativo del presente lavoro abbiamo ritenuto sufficiente, in luogo di rifarci sempre direttamente alle cronache e alle relazioni originali, di far capo al più grande catalogo sismico nazionale del passato recente,

(*) Esso è stato evidenziato anche da sismologi che, in epoca relativamente recente, hanno intrapreso una catalogazione sismica organica: valgano, per tutte, le considerazioni, ben documentate, che fa A. Cavasino in Boll. Soc. Sism. It., XX (1916), pag. $11 \mathrm{sgg}$. 
quello redatto da Mario Baratta e pubblicato nel $1901\left(^{*}\right)$, che riguarda eventi dall'anno 1 dell'era volgare all'anno 1898.

Si tratta, com'é ben noto, di un'opera pregevolissima, fondamentale nel campo della catalogazione sismica anche per i puntuali riferimenti alle fonti storiche. Nella maggior parte dei casi l'autore riporta le indicazioni orarie così come sono annotate nelle cronache da lui consultate, mentre in altri casi traduce nelle ore a.m. e p.m. di tempo medio che s'usavano negli anni in cui egli scriveva (alla fine del secolo $19^{\circ}$ ): così, quel catalogo non è soltanto un repertorio di misure orarie di eventi sismici, ma anche un testo ricco di annotazioni critiche sulle questioni oggetto della nostra discussione.

Allo scopo di confrontare i criteri interpretativi di tali misure che sono stati seguiti dal Baratta con quelli seguiti da studiosi che in tempi assai più vicini si sono occupati di catalogazione sismica, sono stati tenuti presenti anche due cataloghi sismici recenti, e precisamente quello redatto a cura del Comitato Nazionale per l'Energia Nucleare $\left(^{* *}\right)$ e quello redatto a cura dell'Ente Nazionale per l'Energia Elettrica $(* * *)$.

Poiché uno degli scopi pratici del presente lavoro, e segnatamente di questa terza parte di esso, è di attirare l'attenzione sulla corretta interpretazione delle misure temporali storiche, in più di un caso ci capiterà di far rilevare, esplicitamente o implicitamente, interpretazioni orarie non corrette, sia da parte del Baratta, sia da parte di altri. Ma questo fatto, di fronte alla mole e alla qualità dei cataloghi citati, nulla toglie - secondo il nostro convinto avviso - alla capacità e al merito degli studiosi che hanno redatto quei repertori.

(*) M. Baratta, I terremoti d'Italia, Torino 1901. L'opera è divisa in tre parti: I maggiori terremoti italiani (che è il catalogo vero e proprio), pag. 1-668; Distribuzione geografica dei terremoti italiani, pagg. 670-845; Saggio di bibliografia sismica italiana, pagg. 846-950.

$\left.{ }^{* *}\right)$ M.T. Carrozzo, G. De Visintini, F. Giorgetti, E. Iaccarino: General Catalogue of Italian Earthquakes, Comitato Nazionale Energia Nucleare RT/PROT (73) 12, 1973.

${ }^{* * *}$ ) Attualmente disponibile sotto forma di tabulati di calcolatore elettronico. 
Scorrendo il Baratta si ha la convinzione che nel riportare le indicazioni orarie delle fonti storiche egli abbia usato di una grande accuratezza e che in genere non abbia "interpretato " tali indicazioni (salvo quando espressamente detto). Riguardo alla natura delle indicazioni orarie abbiamo sintetizzato la situazione mediante il quadro sinottico esposto nella figura 6, che, a parte alcune minori particolarità, poò ritenersi sufficientemente approssimato per tutta la cronachistica italiana antica.

Sono distinguibili tre periodi in cui la situazione è abbastanza ben definita e due in cui invece, le cose sono piuttosto incerte. Nell'ordine cronologico, ve un periodo iniziale, comprendente l'epoca romana e l'alto medio-evo fino a poco dopo il 1300 (nella fig. 6 compare, per motivi che risulteranno chiari tra breve la data 1329), in cui si usavano ore temporarie; segue un periodo che arriva circa sino alla fine del 1700 , in cui si usavano riferite al tempo attuale, cioè quello del fuso orario $\mathrm{kl}^{\mathrm{h}}$ (in certezza è quello che parte dal 1894, in cui tutte le notizie venivano riferite al tempo attuale, cioè quello del fuso orario $-1^{\text {h }}$ (in sismologia quest'uso ebbe inizio con il $\mathrm{I}^{\circ}$ gennaio 1894).

Notevolmente confusi sono invece il periodo a cavallo del 1400 , in cui si passò dalle ore temporarie a quelle italiane e, soprattutto il periodo di transizione fra le ore italiane e quelle attuali, cioé dalla fine del 1700 al 1894, in cui si trovano mescolate fra loro ore italiane (particolarmente resistenti nell'Italia meridionale $\left({ }^{*}\right)$ e ore a.m. e p.m. sia di tempo vero, sia di tempo medio locale, sia di tempo medio di Roma.

Per la corretta lettura dei dati orari del Baratta, riportiamo una illuminante precisazione che egli fa al termine della premessa alla seconda parte della sua opera (pag. 673) ma evidentemente riferendosi anche alla prima parte, che è il catalogo vero e proprio: "Rispetto alle ore credo utile tener presente che per le date anteriori al 1860 , allorquando non è indicato se siano antimeridiane o pomeridiane, si ritengono contate con il sistema ita-

(*) Nel Baratta, l'ultima indicazione in ore italiane si trova nella descrizione del terremoto calabro del novembre 1869: «...; al 21 novembre ( $1^{h} 30^{m}$ ital. di notte) Cosenza; ...". E' per questo motivo che tale data è posta nella fig. 6 come limite cronologico delle ore italiane. 


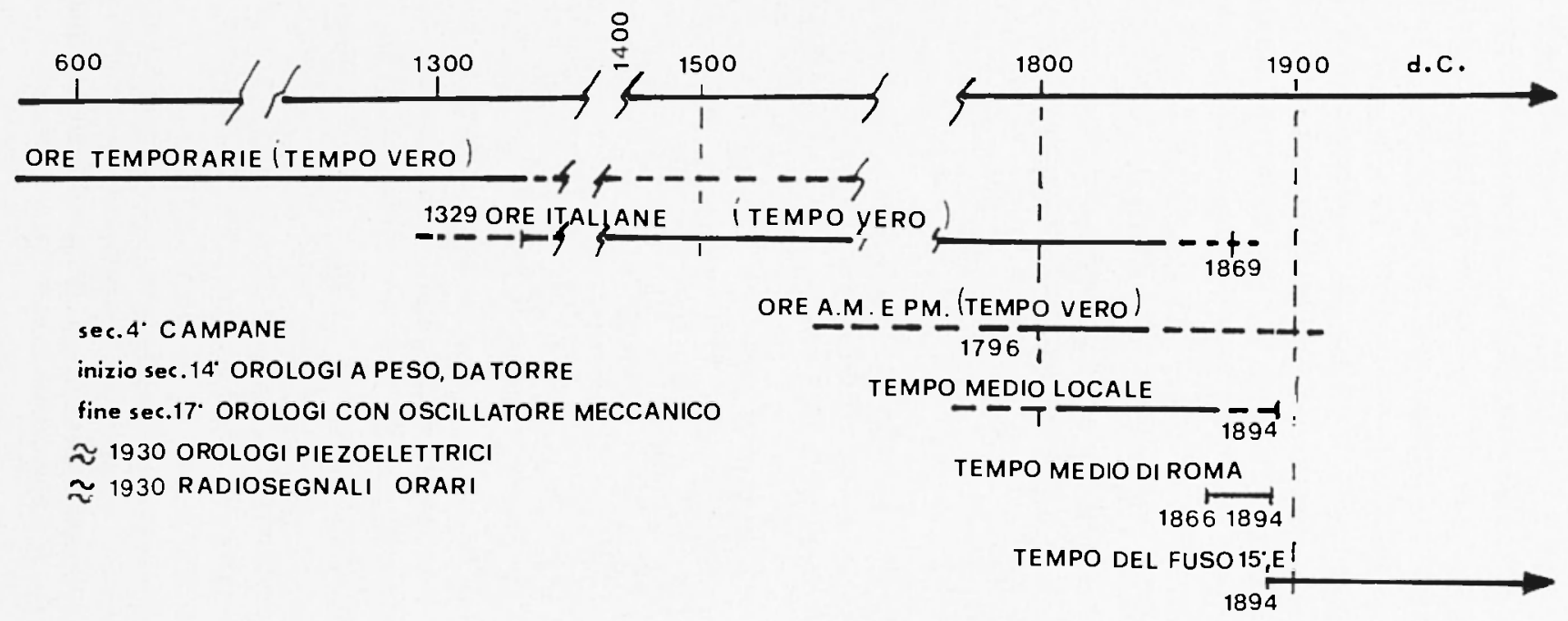

TEMPO UNIVERSALE COORDINATO

Fig. 6 - Quadro sinottico delle scale di tempo in Italia. 
liano: dal 1894 in poi sono espresse in tempo medio dell'Europa centrale e numerate da una mezzanotte all'altra" (*).

Per chiudere questo rapido sguardo generale, è da osservare che indicazioni orarie sia pure sporadiche, compaiono, nel Baratta, già per l'alto medioevo (prima del 1300), ma di esse pochissime sono interpretabili come vere e proprie misure orarie; queste ultime predominano soltanto dal 1400 in poi, con l'affermazione del computo all'taliana. A maggior chiarimento diamo qui di seguito la percentuale in varie epoche delle misure orarie sulle totali indicazioni orarie: anni $1 \div 119920 \%$; secolo $13^{\circ} 30 \%$; secolo $14^{\circ} 50 \%$; secolo $15^{\circ} 85 \%$; secolo $16^{\circ} 90 \%$; secolo $17^{\circ} 97 \%$; poi, praticamente il $100 \%$.

PERIODO DELLE ORE TEMPORARIE E DELLE ORE ITALIANE

\section{Indicazioni orarie medievali.}

Come abbiamo or ora detto nel periodo iniziale che arriva fino al 1300 avanzato si ha a che fare per lo più con indicazioni orarie che sono dei semplici riferimenti temporali.

Ad alcuni di questi riferimenti è però attribuibile un significato cronometrico abbastanza definito: intendiamo le indicazioni di origine romana, di alcuni particolari momenti della giornata, delle quali abbiamo già parlato. Diamo qualche esempio - "al primo canto dei galli" è il gallicinium romano, cioè a tre quarti della notte (fig. 6).

- "all'aurora", "sul far del circa mezz'ora prima dell'alba. giomo $»$, e simili

- «al nascer del dì», e simili all'alba.

- "sul vespro", "sul crepu- circa alla fine del crepuscolo scolo », "all'imbrunire ", serale, e cioè mezz'ora dopo il "sul far della sera", e si- tramonto. mili

(*) Quest'ultima notizia compare già nella prima parte dell'opera, in una nota a pag. 560, per il terremoto del 9 febbraio 1894. 
Altre indicazioni non sono invece riconducibili a momenti abbastanza ben determinati della giornata: "al canto dei galli", che potrebbe essere il gallicinium, ma anche un momento qualunque delle tre ore prima dell'alba; "al mattino", "di sera", e via dicendo; peggio che mai, "di giomo", "di notte".

Per la conversione in tempo universale (quando essa abbia senso) v'é da tener presente che si tratta sempre di tempo solare vero, e che quindi va tenuto conto, nel modo che fu illustrato a suo tempo e che sarà ripreso tra breve, dell'istante del tramonto e dell'equazione del tempo appropriati al luogo e all'epoca dell'anno, nonché della longitudine del luogo.

Ore temporarie. - Le prime vere e proprie misure orarie che si incontrano nel Baratta (escludendo un riferimento orario, tratto da Plinio, per il terremoto che accompagnò la grande eruzione del Vesuvio del 79) sono in ore temporarie e riguardano un terremoto che nell'anno 801 interessò tutta l'Italia centrosettentrionale: sentito a Spoleto "il dì 30 aprile, a $2^{h}$ circa di notte » e a Pontremoli "alla seconda ora della notte del 30 aprile (9-10 pom.) ». Doppiamente interessante, questa citazione, perché in essa v'é anche il risultato della prima conversione in ore p.m. fatta dal Baratta (sulla quale torneremo più avanti). Altre misure dello stesso genere, riguardanti cioè ore notturne, si trovano nel 1117 per un terremoto in alta Italia (" circa le $3^{\text {h }}$ della notte del 3 gennaio, nel 1169 per un terremoto siciliano ( 4 al $4 \mathrm{feb}$ braio circa a un'ora di notte"), nel 1328 a Norcia (" a un'ora di notte I dicembre»), e in anni successivi, alternandosi con più rare misure riguardanti il giorno, quali quelle di cronache del 1249 (Lombardia: "verso le $3^{\text {h }}$ della mattina del 25 luglio"), del 1373 ( In Venezia, al I marzo, verso le $2^{h}$ di giomo»), del 1401 (Belluno: "al 29 gennaio, a $4^{h}$ di giorno »).

Giova osservare che vi sono due espressioni tipiche: "il giorno $\mathrm{x}$, alle ore $\mathrm{x}$ di notte " e " a $\mathrm{x}^{\mathrm{h}}$ (o alla seconda, terza, ... ora) della notte del giorno $\mathrm{x} "$. Queste misure orarie, dell'uno e dell'altro tipo testé evidenziato, sono quasi certamente da prendere come ore temporarie se riferentisi a luoghi che si presume fossero privi di orologi pubblici meccanici: in termini di epoca, 
sino, grosso modo, al 1300 per i grandi centri e sino al 1400 e anche oltre per i centri medi e piccoli. E' invece altamente probabile che dopo queste date, le misure del tipo " ad $\mathrm{x}$ ore della notte " vadano prese come ore italiane; riteniamo che non si possa dire la stessa cosa per le "ore di notte", che incliniamo a ritenere di norma ore temporarie, salvo contraria specifica indicazione, come capita nel tardo Seicento e nel Settecento $\left({ }^{*}\right)$. Peraltro, come si mostrerà più avanti, in molti casi la distinzione fra ore temporarie e ore italiane finisce col non avere una grande importanza pratica.

Discorso analogo va fatto per le (poche) misure in " ore del giorno " e "ore di giorno".

Ore italiane - La prima indicazione in ore italiane il Baratta la trae da un autore del 1700 ( $A$. Mongitore, Istoria cronologica de' terremoti di Sicilia, Palermo 1743), riportando che "sulle $23^{h}$ del 28 giugno [1329] "l'Etna si mise in eruzione; la seconda è nella descrizione del grande terremoto veneto del 1348: " al 25 gennaio 1348 , verso le $23^{h}$ "; seguono via via altre indicazioni da prendersi come ore italiane, se non detto esplicitamente (la prima indicazione esplicita è per il terremoto di Bologna del 2-3 gennaio 1505: "a $9^{n}$ italiane, cioé a $2^{h} 30^{m}$ ant. circa"). Osserviamo che tutte le misure orarie antiche eccedenti la misura massima dell'arco diurno o notturno in Italia, cioé eccedenti, a seconda dell'epoca dell'anno e del luogo, le $12 \div 16^{\mathrm{h}}$, non possono essere che ore italiane (fig. 7): è da escludere infatti che il Baratta abbia fatto una conversione in ore del tipo di quelle nostre attuali, dato che egli ha usato ore a.m. e p.m. per eventi sino a tutto il 1893.

(*) Es. 1697: " al 20 settembre cominciarono a sentirsi in Siena delle scosse di terremoto: le maggiori avvennero circa le $22^{h}\left(4^{h} 1 / 2\right.$ pomt.), le $23^{h}$ (la maggiore) e $4^{h}$ di notte $"$ : la successione del discorso porta ad assimilare l'ultima misura alle due precedenti, che sono in ore italiane. Altro esempio più esplicito: 1727, Noto: " Nel gionno 7 [gennaio], a $7^{\text {hl }} / 2$ ital. di notte $\left(0^{n} 45^{m}\right.$ ant. circa) $)$. 
Precisione delle ore italiane. - Come detto, le ore italiane nacquero, per cosi dire, con gli orologi meccanici pubblici a soneria sui quali si regolavano i campanari e i pochi possessori di orologi privati: a questi orologi pubblici finivano così col riferirsi (talora immediatamente e, più spesso mediatamente) i cronisti.

Gli orologi pubblici erano affidati alle cure di un " temperatore", che aveva il compito, oltre che di "caricarli " quando dovuto, di " regolarli " mediante una meridiana: al mezzogiono indicato da quest'ultima le lancette dell'orologio dovevano indicare un'ora (desunta per ogni giorno dell'anno, da apposite tabelle) tale che esse indicassero poi l'ora zero all'avemaria della sera, e cioé mezz'ora dopo il tramonto del Sole; e poiché l'istante di quest'ultimo può variare di circa un minuto (certe volte in ritardo, certe altre in anticipo) da un giorno al successivo durante l'anno, di altrettanto andava ogni giorno rimesso l'orologio in aggiunta alla rimessa per correggerne l'andamento.

$E^{\prime}$ da tener presente che esistevano, oltre alle normali meridiane a tempo vero, anche meridiane a tempo medio, corrette cioè dell'equazione del tempo e che quindi indicavano il mezzogiorno medio; l'arte orologiaia fu poi in grado, già sul finire del 1600 , di realizzare "orologi a equazione», capaci di indicare contemporaneamente il tempo vero e quello medio. Ma sia questi orologi, sia le meridiane a tempo medio, sia altri più sofisticati dispositivi astronomici per avere il tempo medio locale, erano delle rarità; per controllare gli orologi ci si affidava per lo più a meridiane normali, talché di regola le ore italiane vanno accreditate come una scala di tempo solare vero.

La precisione delle meridiane ordinarie, con stilo lungo intorno al metro, può essere stimata dell'ordine del minuto, certamente maggiore di quella attribuibile intrisecamente alle indicazioni degli orologi (pubblici e privati) dell'epoca di cui stiamo parlando.

Ricorderemo che fino a tutto il 1700 l'Italia ebbe il vanto di possedere, nelle sue maggiori città, meridiane di grande precisione: eccellente fra tutte quella in S. Petronio a Bologna, costruita da G. D. Cassini nel 1655 , in sostituzione di un'altra, poco meno buona, realizzata nello stesso 


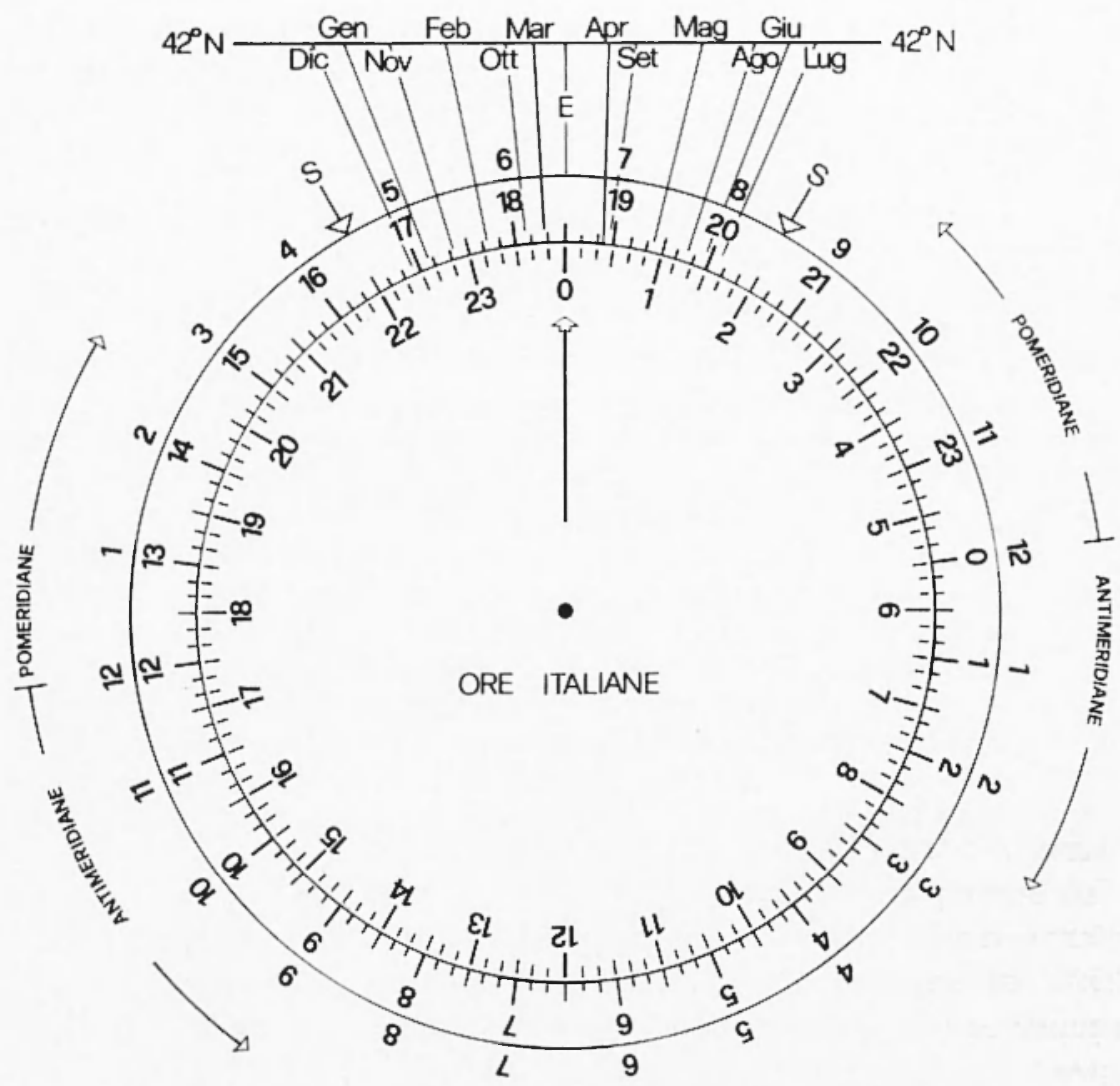

Fig. 7 - Corrispondenza fra ore italiane (all'interno), ore a.m. e p.m., in condizioni equinoziali. $E^{\prime}$ indicata la posizione dello zero delle ore italiane, oltre che agli equinozi (E), anche ai solstizi (S) per le estreme ragioni settentrionali italiane e ( $n$ alto) al 10 di ogni mese per la latitudine di Roma $\left(42^{\circ} \mathrm{N}\right)$.

luogo da I. Danti nel 1575 ("). La meridiana che lo stesso Cassini costrui qualche anno dopo nell'osservatorio di Parigi, pure essa giustamente celebrata, fu una replica, e per di più non altrettanto precisa, di questa

(*) A tale meridiana fu affiancato nel 1758 un orologio a equazione, di ottima fattura, che indicava (usiamo il passato giacché tale orologio da qualche anno è fermo) il tempo vero del meridiano di Bologna, il tempo medio di tale meridiano, il tempo in ore italiane e il tempo medio dell'Europa centrale (questo ultimo aggiunto successivamente). 
di Bologna, la cui esattezza può essere stimata intorno al secondo. Ottime meridiane erano pure in Roma (Torre dei venti in Vaticano, Santa f.Aaria degli Angeli, Obelisco in Piazza S. Pietro), a Firenze (Battistero di S. Giovanni, Santa Maria del Fiore, Santa Maria Novella), a Venezia (S̀dn Marco), a Milano (Duomo).

Duanto agli orologi, è bene tener presente che la regolarizzazione del Irro movimento restò piuttosto approssimativa fino all'applicazione di osillatori a pendolo e a molla-bllanciere. Il regolatore a pendolo, preconizzato da Galilei, entrò nell'uso pratico intorno al 1650: la più antica data autentica è il 1655, e si riferisce a un orologio a pendolo con meccanismo a ruote dentate, costruito a Roma("). Notevoli perfezionamenti furono apportati da $\mathrm{Ch}$. Huygens, il quale nel 1674 fece costruire a Parigi il primo orologio portatile con oscillatore a bilanciere e molla a spirale. Negli orologi sprovvisti di oscillatore la regolarita di marcia era di qualche minuto da un mezzogiorno al successivo; in quelli con uscillatore essa poteva scendere a quelche secondo: ricordiamo che - siamo intorno al 1750 - i celebri cronometri dell'inglese J. Harrison (che a lui fruttarono la vincita di un apposito premio di ben 20.000 sterline istituito dal Governo inglese e alla Marina inglese fruttarono la risoluzione del cosiddetto "problema delle longitudini " e la supremazia nei viaggi oceanici di scoperta) erano capaci di "conservare" il tempo del porto di partenza entro +5 secondi in $5 \div 6$ mesi di navigazione.

Nell'Appendice $\mathrm{C}$ sono riportati alcuni passi riguardanti notazioni cronometriche, tratti da uno studio fatto da un illustre sismologo del 1800 , il padre scolopio Alessandro Serpieri, direttore dell'Osservatorio Ximeniano di Firenze, sul terremoto che sconvolse il riminese nella notte fra il 17 e il 18 marzo 1875. Tale App. ha lo scopo di illustrare quale fosse la situazione sul finire del secolo scorso per quanto riguardava la precisione dei riferimenti orari, in appoggio a pertinenti considerazioni che faremo più avanti. La lettura di quei passi è utile anche in rapporto alle considerazioni che stiamo facendo ora, in quanto mostra che la maggior parte delle misure orarie là riportate appare affetta da un errore di qualche minuto: si noti che ci si trovava già nell'epoca in cui vigeva il tempo medio di Roma, che veniva "distribuito" in tutt'Italia mediante i collegamenti telegrafici con gli uffici postali e le stazioni ferroviarie. E' facile

${ }^{*}$ E. Von Bassermann-Jordan, Uhren, $2^{\circ}$ ediz. franc., Fribuurg $(\mathrm{CH})$, pag. 372. 
arguire una situazione ben peggiore nei secoli precedenti, nei quali, oltre a tutto, la regolarità di marcia degli orologi nelle 24 ore fra un controllo alla meridiana e il successivo era certamente scarsa.

Con una certa dose di ragionevole ottimismo, siamo propensi ad attribuire un errore intrinseco di $+5 \div 10^{\mathrm{m}}$ alle misure date in ore italiane dal finire del 1700 in poi e di $+10 \div 20^{\mathrm{m}}$ per quelle date in epoche anteriori (fra il 1400 e il 1800).

In realtà, v'è ancora un fattore di cui si deve tener conto.

Quanto ora detto ha senso soltanto nel caso di letture fatte sugli orologi al momento del verificarsi dell'evento.

Spesso, invece, la cronaca è fatta in base al racconto dei protagonisti della vicenda, i cui riferimenti temporali erano, parlando in termini generali, i rintocchi orari degli orologi civici. Questi venivano dati, con i primi orologi, soltanto di ora in ora; più tardi (e a cominciare dai centri abitati di maggiore importanza) furono dati anche ai quarti d'ora.

Ciò è ben evidenziato dal fatto che nelle cronache più antiche per molto tempo le misure venivano espresse in ore intere. accompagnate per di più da locuzioni di approssimazione (circa le ore $x$ ", "verso $x$ ore ", e simili). La prima misura oraria frazionaria ( $4^{h} 30^{m}$ di notte ") è, nel Baratta, del 1455 (terremoto del 5 dicembre, Bologna); successivamente, se ne hanno nel 1511 (26 marzo, Friuli: "Fra le $20^{h}$ e le $20^{h} 30^{m}$ "), nel 1542 (13 giugno, Mugello: "Circa le $6^{h_{1}}$ del mattino "), nel 1558 (13 aprile, Siena: "Circa le $14^{h} 30^{m} "$ ), e poi via via più frequentemente. I quarti d'ora cominciano ad apparire verso il 1700; la pruma indicazione è del 1694 ( 8 settembre, Avellino: "verso le 17/3/4 ital. "). Orbene, viene spontaneo di attribuire a queste misure un "errore di lettura " con un ordine di grandezza pari all'ampiezza della graduazione: precisamente, $+30^{\mathrm{m}}$ per le misure "all'ora", $\pm 7,5^{\mathrm{m}}$ per quelle "al quarto d'ora".

Complessivamente, e cioé tenendo conto di tutte le cause d'errore, si può formulare il seguente criterio di massima per l'entità dell'errore da attribuire alle misure in ore italiane: $+30^{\mathrm{m}}$ per le misure all'ora, $+15^{\mathrm{m}}$ per quelle al quarto d'ora, beninteso a meno di probanti argomenti in favore di una precisione mi- 
gliore, come potrebbe essere, per es., la notizia dell'ancoraggio delle misure a controlli fatti da una buona specola ( $\left.{ }^{*}\right)$.

$\mathrm{E}^{\prime}$ evidente che di fronte a errori assoluti di quest'ordine di grandezza diminuisce la rilevanza della questione (dianzi accennata a proposito del controllo meridiano degli orologi) se la regolazione degli orologi venisse fatta in tempo vero, come tutto porta a pensare, o non piuttosto in tempo medio, come in qualche caso potrebbe essere: la differenza fra i due tempi, cioè l'equazione del tempo, non supera infatti, come s'è detto, pochi minuti per gran parte dell'anno (soltanto fra metà gennaio e metà marzo, e in ottobre e novembre, oscilla fra $10^{\mathrm{m}}$ e circa $16^{\mathrm{m}}$ ).

Conversione delle ore italiane in tempo universale.

Il procedimento è il seguente:

1) si determinano, con appropriato grado di approssimazione $\left({ }^{* *}\right)$ le coordinate geografiche del luogo $A$ cui la misura si riferisce, $A \in\left(\varphi_{A}, \lambda_{A}\right)$;

2) si determina l'istante di tempo solare vero corrispondente all'inizio del "giorno all'italiana » considerato, cioè l'istante della fine del crepuscolo solare, $\left(t_{v}\right)_{c}$, dato dalla [6];

3) si trasforma in tempo medio il tempo di cui sopra, mediante l'equazione del tempo $E$, e il tempo medio in tempo civile, aggiungendo $12^{\mathrm{h}}$; queste due rasformazioni si effettuano mediante la [11], ottenendosi così l'istante iniziale di tempo civile del giorno all'italiana, $\left(t_{c}\right)_{i}$, per il luogo e l'epoca dati;

(*) Già sul finire del 1600 buone specole astronomiche esistevano in tutte le città sede di università, e anche in altre. Osservatori astronomici nel significato odierno della locuzione si svilupparono da queste specole nel 1700; ricordiamo la data di fondazione di Osservatori tuttora esistenti: Bologna 1712, Torino (Pino) 1759, Milano (Brera) 1760, Padova 1761, Roma (Collegio romano) 1787, Palermo 1790, Firenze 1807, Napoli (Capodimonte) 1812, (G. Abetti, Storia dell'Astronomia, Firenze 1949).

$\left.{ }^{* *}\right)$ In genere è sufficiente l'approssimazione al decimo di grado per la latitudine, al centesimo di ora per la longitudine. 
4) si aggiunge a tale istante iniziale il tempo dell'evento in ore italiane $t_{1}$, ottenendosi il tempo civile (locale) dell'evento in $A,\left(t_{c}\right)_{A}$;

5) si ottiene infine l'istante dell'evento in tempo universale, TU, tenendo conto della longitudine $\lambda_{A}$ di $A$, espresso in ore (che, nel caso di località italiane, tutte ad est di Greenwich, compare sempre in detrazione);

6) si esprime il risultato in ore e minuti, con arrotondamento al quarto d'ora).

In sintesi:

$$
\begin{aligned}
& T U=\left\{\left[\left(t_{v}\right)_{c}-E+12^{h}\right]+t_{i}\right\}+\lambda_{A}, \\
& \mathrm{TU}=\left[\left(t_{c}\right)_{i}+t_{i}\right]-\lambda_{A}, \\
& T U=\left(t_{c}\right)_{A}-\lambda_{A}
\end{aligned}
$$

dove al risultato vanno tolte $24^{\mathrm{h}}$ se esso supera la durata di un giorno.

Per facilitare il calcolo, nell'App. D sono riportate latitudine e longitudine delle città capoluogo di provincia, e nel'App. E i valori dell'istante d'inizio $\left(t_{c}\right)_{i}$, in tempo civile locale, delle ore italiane, nonché quelli dell'equazione del tempo $E$, di decade in decade durante l'anno e di grado in grado fra $37^{\circ}$ e $47^{\circ}$ di latitudine $\mathrm{N}$ (corrispondenti alle estreme regioni meridionali e settentrionali del nostro paese) $\left(^{*}\right)$.

(*) Poiché le coordinate celesti e terrestri, nonché l'equazione del tempo, sono soggette a variare (sia pure di molto poco) nel corso degli anni, i valori dati nell'App. E vanno intesi come valori medi per le epoche indicate, con l'approssimazione appropriata per i calcoli di conversione di misure orarie del passato. 
Interpretazione e accuratezza delle ore temporarie. - Come s'é accennato, nel periodo in cui le indicazioni cronometriche venivano date prevalentemente in ore italiane, si incontrano ancora qua e là misure in ore temporarie derivanti dalla ripartizione in 12 parti uguali dell'arco diurno (ore di giorno) e dell'arco notturno (ore di notte).

La via più conveniente per l'interpretazione di queste misure è di riportarsi alle corrispondenti ore italiane, e da queste poi, come abbiamo mostrato, al tempo universale.

Per passare da una misura data in ore temporarie $t_{t}$, alla corrispondente misura $t_{\imath}$ in ore italiane valgono le relazioni seguenti:

$t_{i}=\frac{d_{n}}{12} t_{t}-0,5^{\mathrm{h}}=\left(\frac{24-d_{g}}{12}\right) t_{t}-0,5^{\mathrm{h}}$ per le ore di notte

$t_{i}=23,5^{\mathrm{h}}-d_{g}\left(1-\frac{t_{t}}{12}\right)$

per le ore di giorno

dove $d_{n}, d_{g}$ sono le durate dell'arco notturno e diurno, rispettivamente, per le quali si fa capo alle [4], [5] (*).

Nelle [21] si assume che il computo delle ore di notte inizi al tramonto del Sole e quello delle ore di giorno inizi all'alba.

La durata del giorno $d_{g}$ varia in Italia (alle alte latitudini) fra circa $8^{\mathrm{h}}$ (intorno al solstizio d'inverno) e circa $16^{\mathrm{h}}$ (intorno al solstizio d'estate); corrispondentemente per la durata della notte. Così, in periodi solstiziali, le ore temporarie possono essere

(*) E, da queste, alla [3]. Più comodamente, possono essere usati dati dell'App. E, con i quali s'ottengono risultati meno precisi, ma accettabili per $\mathrm{i}$ calcoli qui in discussione.

Precisamente, si tratta di convertire l'istante iniziale delle ore italiane $\left(t_{c}\right)_{i}$, dato nell'App. E, da tempo civile in tempo medio (il che si ottiene togliendo da esso $12^{\mathrm{h}}$ ) e poi in tempo vero (aggiungendo algebricamente l'equazione del tempo $E$, anch'essa data nell'App. E); si ottiene cosi l'istante di tempo vero dell'avemaria della sera $\left(t_{v}\right)_{c}$, dal quale, sot- 
di circa un terzo più lunghe oppure più corte rispetto alle ore ordinarie. Ciò significa che se, per incertezza delle fonti o per amore di semplicità, ci si inducesse a considerare le ore di notte $\left({ }^{*}\right)$ come se fossero ore italiane, si verrebbe ad aggiungere all'errore intrinseco della misura un altro errore che, praticamente nullo in epoche sufficientemente lontane dai solstizi, potrebbe raggiungere il 30\% circa nei mesi solstiziali, e quindi superare l'errore intrinseco.

Quest'ultimo errore è senz'altro maggiore di quello attribuibile alle ore italiane. Il fatto stesso che ancora in epoca avanzata $\left({ }^{* *}\right)$ ci si servisse di ore temporarie fa pensare che nei luoghi interessati non esistessero buoni orologi meccanici, supposizione suffragata dal fatto che generalmente le misure temporarie sono date all'ora e non in ore e mezz'ore o quarti d'ora: è da presumere quindi che in quei luoghi il servizio del tempo fosse piuttosto al disotto dello standard dell'epoca.

Per questo motivo siamo inclini ad attribuire a queste indi-

traendo $0,5^{\mathrm{h}}$, si ha l'istante di tempo vero del tramonto, $\left(t_{v}\right)_{0}$ : raddoppiando quest'ultimo, si ha infine la durata dell'arco diurno $d_{b}$, in funzione della quale possono essere usate le [21].

Diamo un esempio: 31 gennaio, lat. $42^{\circ} \mathrm{N}$.

avemaria della sera, tempo civile (App. E) $\left(t_{c}\right)_{i}$

17,70

tempo medio $\left(-12^{\mathrm{h}}\right)$

5,70

equazione del tempo

(App. E) $E$

$-0,23$

avemaria della sera, tempo vero

$\left(t_{1}\right)_{c}$

5,47

tramonto,

tempo vero

$\left(-0,5^{\mathrm{h}}\right)$

$\left(t_{v}\right)_{o}$

4,97

durata arco diurno

(x 2$) \quad d_{\varepsilon}$

$9,94 \approx 10^{\mathrm{h}}$

Si raccomanda vivamente di condurre questi calcoli, e altri di natura simile, con misure tutte espresse in ore, al fine di evitare errori che con facilità si commettono se si esprimono le misure orarie nell'ordinaria forma complessa, e cioè in ore e minuti.

(*) $\mathrm{Ci}$ riferiamo a queste perché esse sono molto più frequenti che non le ore di giorno. Per queste ultime, il discorso, comunque, è analogo.

(**) Nel Baratta, misure interpretabili come ore temporarie di notte, compaiono ancora nella seconda metà del 1600 . 
cazioni cronometriche un errore non minore di $+45^{\mathrm{m}}$, salvo, beninteso, il reperimento, di caso in caso, di probanti elementi in favore di una migliore precisione.

Cosi :stando le cose (a meno che il carattere dell'indagine richieda una conversione più precisa) riteniamo che in vari casi le prime ore di notte $\left(2^{\mathrm{h}} \div 3^{\mathrm{h}}\right)$ possano essere trattate come se fossero ore italiane, attribuendo alla misura un errore di $\pm 35^{\mathrm{m}}$. Un errore assoluto di tale ampiezza rende poco rilevante il fatto di chiarire se il computo delle ore temporarie veniva fatto dal tramonto, come tutto fa pensare, oppure dall'avemaria della sera, come per le ore italiane.

Analisi di conversioni orarie presenti nel catalogo del $\mathrm{Ba}$ ratta. - In un certo numero di casi (una trentina), nel catalogo Baratta è data, accanto alla misura in ore temporarie o italiane, la corrispondente misura in ore antimeridiane o pomeridiane, calcolata (presumibilmente) dallo stesso Baratta.

Sorge subito la questione se queste misure "convertite" debbano essere intese in tempo vero, tempo medio locale, tempo medio di Roma, tempo medio dell'Europa centrale: tempi, questi, che venivano usati tutti nel periodo in cui il Baratta attese alla compilazione e alla pubblicazione della sua opera.

Fra i tempi ora nominati, il tempo medio dell'Europa centrale è il meno probabile, e, a nostro parere, non va preso in considerazione anche per una ragione di carattere formale, e cioè che esso, almeno ufficialmente, contava le ore del giorno da $1^{\mathrm{h}}$ a $24^{\mathrm{h}}$, e non in forma duodecimale, come viceversa sono le misure date dal Baratta per eventi sino a tutto il 1893.

Per formarci un'idea su tale questione, abbiamo effettuato la conversione delle misure già considerate dal Baratta, e ciò sia in tempo medio locale, sia in tempo medio di Roma, sia in tempo universale, secondo le procedure dianzi esposte.

Diamo qualche esempio.

Esempio 1. 1456. Dicembre 5. Napoletano.

Nel Baratta, dopo un'analisi critica di varie notizie discordanti sulla data (un'altra grande scossa vi fu il giorno 30), relativamente ai tempi in cui la scossa fu sentita a Napoli si legge (pag. 66): 
L'ambasciatore senese scriveva in data 7 dicembre: "a di 4 de questo, sonate le XI hore venne uno terremoto...". Ercole marchese di Ferrara anche egli dava partecipazione dellavvenimento con una lettera in cui dice: "sabato quarto giomo dello stesso mese sino alla domenica verso le dodici ore avvenne un terremoto..." ". L'ambasciatore milanese Antonio da Trezzo, dando notizia della catastrofe a Francesco Sforza, incomincia cosi la sua relazione: "Sabato passato de nocte, circa le due hore, venendo la domenica, fò qua el magiore terremoto...". Ed infine un manoscritto sincrono della Università di Pavia pubblicato dal Romano [G. Romano, in Arch. Stor. per le provincie Napoletane, anno XIII, fasc. IV] concorda con ciò pienamente, giacché riferisce che "MCCCCLVI a dy quatro de dexembre tra le $X$ e XI hora e durò per un decimo dora fu in lo Reame un terremoto...". Adunque concludendo, la scossa disastrosa avvenne netla notte del 4-5 dicembre tra le ore 10 e 11 e durò circa 6 minuti.

Così il Baratta. Converrà ripetere le misure cronometriche: tre sono abbastanza coerenti fra loro, e precisamente: "sonate le $X$ hore", "verso le dodici hore", "tra la X e XI hora", mentre una quarta è discordante, e precisamente "sabato passato de nocte, circa le due hore venendo la domenica». Lasciando per il momento da parte questa ultima misura, leggiamo le prime tre - seguendo il Baratta - come 10 ore e mezza, e proviamo a interpretarle dapprima come ore temporarie e poi come ore italiane.

Per il luogo e per l'epoca dati, l'App. E dà per l'inizio delle ore italiane $\left(t_{c}\right)_{i}$ l'istante $16,96^{\mathrm{h}}$ di tempo civile e per l'equazione del tempo $E$ il valore $+0,15^{\mathrm{h}}$. Sulla falsariga di quanto mostrato poco sopra, si ottiene per la durata dell'arco diurno $d_{\varepsilon}$ il valore $9,2^{\mathrm{h}}$; la prima delle [21] dà allora, in corrispondenza a 10,5 ore di notte, 12,45 ore italiane. Aggiungendo queste all'istante dell'ora zero, cioè a 16,96, si ha (a meno di 24h) $5,41^{\mathrm{h}}$, cioè $5^{\mathrm{h}} 24,6^{\mathrm{m}} \approx 05^{\mathrm{h}} 25^{\mathrm{m}}$ di tempo medio locale; detraendo (App. D) la longitudine di Napoli rispetto a Roma $\left(0,12^{\mathrm{h}} \mathrm{E}\right)$ e rispetto a Greenwich $\left(0,9^{\mathrm{h}} \mathrm{E}\right)$, si hanno i ltempo medio di Roma, $5,29^{\mathrm{h}}=5^{\mathrm{h}} 17,4^{\mathrm{m}} \approx 05^{\mathrm{h}} 15^{\mathrm{m}}$, e quello universale, $4,46^{\mathrm{h}}=4^{\mathrm{h}} 27,6^{\mathrm{m}} \approx 04^{\mathrm{h}} 30^{\mathrm{m}}$.

Interpretando le $10,5^{\mathrm{h}}$ come ore italiane, si ottiene, aggiungendo tale misura a $16,96^{\mathrm{h}}$ e poi detraendo le longitudini, come fatto ora: $\approx 03^{\mathrm{h}} 30^{\mathrm{m}}$ di tempo medio locale, $\approx 03^{\mathrm{h}} 20^{\mathrm{m}}$ di tempo medio di Roma, $\approx 02^{\mathrm{h}} 30^{\mathrm{m}}$ di tempo universale.

A questo punto, se non esistessero altri elementi di giudizio, si dovrebbe propendere per l'interpretazione delle 10 ore e mezza come ore temporarie, che appare ben coerente con l'epoca. Esiste però la quarta misura, ciò̀ le $2^{\mathrm{h}}$ di notte, che, a priori, merita altrettanta fiducia delle altre.

Questa misura dà, se interpretata sia in ore temporarie sia in ore italiane, $\approx 19^{\mathrm{h}} 00^{\mathrm{m}} \mathrm{TU}$, troppo distante dalle altre tre. Secondo il nostro 
parere, essa va intesa come una locuzione indicante due ore (quasi certamente temporarie) dall'inizio della domenica, e cioè dalla mezzanotte ${ }^{*}$ ): a conti fatti, $\approx 01^{\mathrm{h}} 30^{\mathrm{m}} \mathrm{TU}$, non troppo distanti dalle 0230 corrispondenti all'interpretazione delle altre misure come ore italiane.

In definitiva - l'accordo con quanto dice il Baratta nell'avvertenza (citata in precedenza) a pag. 673 della sua opera - già a partire dal 1400 c da presumere che nei casi incerti debba prevalere l'interpretazione in ore italiane, anche in presenza di espressioni del tipo "ore di notte" o "ore della notte" $\left(^{* *}\right)$.

Esempio 2. 1687. Aprile 25-26. Amalfi.

Dal Baratta (pag. 152):

... nel Salernitano alla mezzanotte del 25.... ... le $5^{\text {hit } / 2}$ ital. corrispondono appunto alla mezzanotte...

Dall'App. E si trova $\left(t_{c}\right)_{i}=19,28^{\mathrm{h}} \approx 19 \mathrm{~h} 17^{\mathrm{m}}$; aggiungendo $t_{i}=5,5^{\mathrm{h}}$ italiane si ha per il tempo medio locale $24,78^{\mathrm{h}}=00^{\mathrm{h}} 46,8^{\mathrm{m}} \approx 00^{\mathrm{h}} 45^{\mathrm{m}}$; tenendo conto delle longitudini (App. D) si ha per il tempo medio di Roma $0,63^{\mathrm{h}}=00^{\mathrm{h}} 37,8^{\mathrm{m}} \approx 00^{\mathrm{h}} 40^{\mathrm{m}}$ e per il tempo universale $23,80^{\mathrm{h}}=23^{\mathrm{h}} 48^{\mathrm{m}} \approx$ $23^{1 / 2} 50^{\mathrm{m}}$. La notevole divergenza rispetto al tempo medio indicato dal Baratta (me\%anotte invece di mezzanotte e $45^{\mathrm{m}}$ ) è dovuta al fatto che egli ha preso per l'istante di inizio delle ore italiane $18^{\mathrm{h}} 30^{\mathrm{m}}$ (anziché $19^{\mathrm{h}} 17^{\mathrm{m}}$ ), come se si fosse in condizioni equinoziali.

\section{Esempio 3. 1730. Maggio 12. Norcia.}

Dal Baratta (pag. 216):

$1 l 12$ maggio, alle ore 101/4 ital. (6 $30^{\mathrm{m}}$ ant. circa) una scossa...: alle $19^{i t} 1 / 2\left(2^{1 / 3} / 4\right.$ pom.) una replica violentissima arrecò nuovi danni.

Dall'App. E si trova l'ora d'inizio delle ore italiane $19,66^{\mathrm{h}} \approx 19^{\mathrm{h}} 40^{\mathrm{m}}$ che, ovviamente, è la stessa per le due misure; stranamente, per la prima conversione del Baratta tale ora iniziale risulta $20^{\mathrm{h}} 15^{\mathrm{m}}$, mentre per la seconda risulta $19^{\mathrm{h}} 15^{\mathrm{m}}$, cioè un'ora esatta in meno. Aggiungendo a $19,66^{\mathrm{h}}$ le misure in ore italiane e tenendo poi conto delle longitudini (App. D), si ottengono, nell'ordine per le due misure, i tempi medi locali $05^{\mathrm{h}} 5^{\mathrm{m}} \mathrm{m}$ e $15^{\mathrm{h}} 10^{\mathrm{m}}$, i tempi medi di Roma $05^{\mathrm{h}} 50^{\mathrm{in}}$ e $15^{\mathrm{h}} 05^{\mathrm{m}}$, i tempi universali $05^{\mathrm{h}}$ $00^{\mathrm{m}}$ e $14^{\mathrm{h}} 15^{\mathrm{m}}$.

(*) Secondo l'uso ecclesiastico, e anche secondo l'uso francese: è probabile che quest'ultimo fosse consuetudinario per l'ambasciatore di $\mathrm{Mi}$ lano a Napoli.

(**) Per questo motivo, nella successiva tab. II abbiamo interpretato le $" 5^{h}$ di notte" nella descrizione del terremoto friulano del 6 giugno 1794 direttamente come ore italiane. 
Tabella II

ELEMENTI RIGUARDANTI MISURE ORARIE ANTICHE CONVERTITE

\begin{tabular}{|c|c|c|c|c|c|c|c|c|c|c|}
\hline \multicolumn{2}{|c|}{ Evento } & \multicolumn{2}{|c|}{ Misure del Baratta } & \multicolumn{2}{|c|}{ Inizio ore italiane } & \multirow{2}{*}{$\begin{array}{l}\text { t.m. } \\
\text { locale } \\
\text { (IV) }\end{array}$} & \multirow{2}{*}{ 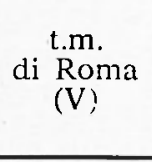 } & \multicolumn{3}{|c|}{ Tempo Universale } \\
\hline Data & Luogo & antiche & $\begin{array}{c}\text { conv. } \\
\text { (I) }\end{array}$ & $\begin{array}{c}\mathrm{B} \\
\text { (II) }\end{array}$ & $\begin{array}{c}\text { DM } \\
(\mathrm{III})\end{array}$ & & & DM & ENEL & CNEN \\
\hline \multicolumn{11}{|l|}{ ore temporarie } \\
\hline 80130 aprile* & Pontremoli & $2^{\mathrm{a}}$ ora di notte & 2130 & & & 2050 & 2100 & 2010 & & 20 \\
\hline 142510 agosto* & Ferrara & $I^{\text {h }}$ di notte & 2030 & & & 1950 & 1950 & 1900 & 19 & 2030 \\
\hline 14565 dicembre & Napoli & tra le X e XI hora & $0200 ?$ & & & 0525 & 0515 & 0430 & & 0430 \\
\hline \multicolumn{11}{|l|}{ ore italiane } \\
\hline 14565 dicembre & Napoli & tra le $\mathrm{X}$ e XI hora & $0200 ?$ & & 1700 & 0330 & 0320 & 0230 & 22 & 0430 \\
\hline 15053 gennaio* & Bologna & $9^{\text {h }}$ italiane & 0230 & 1730 & 1710 & 0210 & 0215 & 0125 & 01 & $\begin{array}{l}0130 \\
\text { (del 2) }\end{array}$ \\
\hline 154213 giugno & Mugello & $6^{\mathrm{h}} 1 / 2$ matt. & 0200 & 1930 & 2010 & 0240 & 0245 & 0155 & 9 & 01 \\
\hline 155126 settembre & Firenze & $23^{h}$ circa & 1730 & 1830 & 1800 & 1700 & 1700 & 1610 & 16 & 1630 \\
\hline 155813 aprile & Siena & $14^{\mathrm{h}} 30^{\mathrm{m}}$ & 0930 & 1900 & 1920 & 0950 & 0955 & 0905 & 8 & 0830 \\
\hline 15973 agosto* & Scarperia & alle $4^{h} \quad 1 / 2$ & 2400 & 1930 & 1950 & 0020 & 0025 & 2335 & 23 & 23 \\
\hline 162730 luglio & Foggia & $16-17^{\mathrm{h}}$ ital. & 1200 & 1930 & 1945 & 1215 & 1205 & 1115 & 1530 & 11 \\
\hline 167824 marzo & Siena & ore 17 & 1230 & 1930 & 1845 & 1145 & 1150 & 1100 & 16 & 1130 \\
\hline 168725 aprile* & Amalfi & $5^{\text {h }} 1 / 2$ ital. & 2400 & 1830 & 1915 & 0045 & 0040 & 2350 & 23 & 23 \\
\hline 169720 settembre & Siena & $22^{h}$ & 1630 & 1830 & 1825 & 1625 & 1630 & 1540 & 15 & 15 \\
\hline 20 settembre & Siena & $23^{h}$ & 1730 & 1830 & 1825 & 1725 & 1730 & 1640 & 16 & 16 \\
\hline 27 settembre & Siena & $15^{\mathrm{h}}$ & 0930 & 1830 & 1815 & 0915 & 0920 & 0830 & 830 & 830 \\
\hline 172411 dicembre & Siena & circa le $20^{\mathrm{h}}$ & 1300 & 1700 & 1655 & 1255 & 1300 & 1210 & 12 & 13 \\
\hline 17277 gennaio* & Noto & $7^{\text {h }} 1 / 2$ ital. & 0045 & 1715 & 1725 & 0055 & 0045 & 2355 & 0630 & 24 \\
\hline 173028 marzo & Massa & circa le $7^{\mathrm{h}}$ & 0300 & 2000 & 1850 & 0150 & 0200 & 0110 & 6 & 2 \\
\hline \multirow[t]{2}{*}{173012 maggio } & Norcia & $10^{\mathrm{h}} \mathrm{t} / \mathrm{A}^{\mathrm{s}}$ ital. & 0630 & 2015 & 1940 & 0555 & 0550 & 0500 & 0915 & 0530 \\
\hline & & $19^{\mathrm{h}} \mathrm{t} / 2$ & 1445 & 1915 & 1940 & 1510 & 1505 & 1415 & 1830 & 13 \\
\hline 17406 marzo & Garfagnana & $12^{\mathrm{h}} 1 / 4 \mathrm{ital}$. & 0630 & 1815 & 1825 & 0640 & 0645 & 0555 & 0530 & 0515 \\
\hline 174611 luglio & Barga & $18^{h}$ & 1400 & 2000 & 2010 & 1410 & 1420 & 1330 & 13 & 13 \\
\hline 176215 aprile & Mugello & $23^{\mathrm{h}}$ & 1800 & 1900 & 1910 & 1810 & 1815 & 1725 & 2230 & 17 \\
\hline \multirow{2}{*}{$\begin{aligned} & 7 \text { febbraio } \\
9 & \text { febbraio }\end{aligned}$} & Genova & $11^{\mathrm{h}}$ ital. & 0415 & 1715 & 1740 & 0440 & 0455 & 0405 & 0315 & 0315 \\
\hline & Genova & $21^{\mathrm{h}} 3 / 4$ ital. & 1530 & 1745 & 1740 & 1525 & 1540 & 1450 & 1530 & 15 \\
\hline 17791 g्रingno* & Bolcu:... & $4^{\text {h }} 1 / 2$ ital. & 2400 & 1930 & 2005 & 0035 & 0040 & 2350 & 23 & 24 \\
\hline 17831 marzo & Messina & 8,30 ital. & 0215 & 1745 & 1825 & 0255 & 0240 & 0150 & 0130 & 0130 \\
\hline 178625 dicembre & Rimini & dopo $9 \mathrm{~h}$ ital. & 0200 & 1700 & 1655 & 0155 & 0155 & 0105 & 01 & 01 \\
\hline 178820 ottobre & Tolmezzo & $4^{\text {h }} 1 / 2$ ital. & 2200 & 1730 & 1730 & 2200 & 2200 & 2110 & $\begin{array}{l}2300 \\
\quad(\text { del 19) }\end{array}$ & 2130 \\
\hline 1 novembre & Tolmezzo & $15^{\mathrm{h}}$ & 0815 & 1715 & 1710 & 0810 & 0810 & 0720 & 0715 & 0715 \\
\hline 5 novembre & Tolmezzo & ore $14 \begin{array}{l}\text { (Bar. dà, } \\
\text { errando) }\end{array}$ & 0405 & & 1710 & 0710 & 0705 & 061 & 0305 & 0305 \\
\hline 17946 giugno* & Friuli & $5^{\text {h }}$ di notte & 0100 & 2000 & 2010 & 0110 & 0110 & 0020 & $\begin{array}{l}0000 \\
(\text { del 7) }\end{array}$ & 0015 \\
\hline 180526 luglio* & Napoli & $2^{\mathrm{h}} 20^{\mathrm{m}}$ ital. & 220140 & 1942 & 1948 & 2208 & 2201 & 2111 & 210140 & 210140 \\
\hline 181714 marzo* & Messina & $05^{\mathrm{h}} 50^{\mathrm{m}}$ it. & 2350 & 1800 & 1830 & 0020 & 0010 & 2320 & 2250 & 2250 \\
\hline 182921 maggio* & Albano & $4^{\text {h }}$ ital. & 2348 & 1948 & 1945 & 2345 & 2345 & 2255 & 2248 & 2248 \\
\hline
\end{tabular}


Esempio 4. 1805. Luglio 26. Molise.

Dal Baratta (pag. 318):

A $2^{h} 20^{m}$ ital. del 26, ossia a $10^{h}$ Im $^{m} 40^{\text {s }}$ pom. (t.v.) si sentì in Napoli... l'ora di Napoli (10 $1^{m} 40^{\text {s }}$ p.t.v.) fu dedotta da un pendolo astronomico fermatosi appunto in detto istante:...

Questa volta si tratta di una conversione che il Baratta fa al contrario,, per così dire: egli infatti parte dal tempo vero $\left(^{*}\right)$, che è il dato sperimentale, e calcola la corrispondente ora italiana. Poiché la misura è data al secondo, con un errore probabile di qualche secondo, svolgiamo i calcoli con la migliore precisione deducibile dai valori delle App. D, E $(* *)$ :

tempo vero

—equaz. del tempo

tempo medio locale

long. da Greenwich

tempo universale

longitudine da Roma

tempo medio di Roma $22^{\mathrm{h}} 01^{\mathrm{m}} 40^{\mathrm{s}}=22,027.8^{\mathrm{h}}$

$$
+0,105
$$$$
22,132.8=22^{\mathrm{h}} \quad 07^{\mathrm{m}} 58,1^{\mathrm{s}} \approx 22^{\mathrm{h}} 08^{\mathrm{m}}
$$$$
-0,951.0
$$

$$
\begin{aligned}
& 21,181.8=21^{\mathrm{h}} 10^{\mathrm{m}} 54,5^{\mathrm{s}} \approx 21^{\mathrm{h}} 11^{\mathrm{m}} \\
& -0,119.0
\end{aligned}
$$$$
22,013.8=22^{\mathrm{h}} 00^{\mathrm{m}} 49,7^{\mathrm{s}} \approx 22^{\mathrm{h}} 01^{\mathrm{m}}
$$

L'App. E dà per l'ora d'inizio delle ore italiane il valore $19,80\left(19^{\mathrm{h}} 48^{\mathrm{m}}\right)$, che sottratto dal tempo medio locale dà, per le ore italiane, il valore 2,33 ciò̀ le $2^{\mathrm{h}} 20^{\mathrm{m}}$ del Baratta.

I risultati di questi calcoli di verifica sono raccolti nella tabella II. In questa sono esposti, ordinatamente: la data dell'evento (ovviamente secondo il Baratta); il luogo dell'evento (che di norma non è l'epicentro del sisma); le citazioni del Baratta relative alla misura antica e alla misura corrispondente secondo il Baratta, scritta in notazione moderna; l'istante di inizio delle ore italiane in quel giorno e in quel luogo, secondo il $\mathrm{Ba}$ -

(*) In realtà, si tratta del tempo vero aumentato di $12^{\text {h }}$ cioè contato a partire dalla mezzanotte anziché dal mezzogiorno; per semplicità, abbiamo mantenuto quest'uso anche nel seguito.

(**) Per conversioni di tempi veri o medi dati con l'approssimazione del secondo conviene, per conservarne la precisione delle misure di partenza, fare capo, per l'equazione del tempo, ad Annuari astronomici del- 
ratta (colonna B) e secondo noi (colonna DM); il tempo medio locale e quello medio di Roma dell'evento, secondo i nostri calcoli; il tempo universale dell'evento, secondo i nostri calcoli (colonna DM) e secondo quanto è riportato nei cataloghi ENEL e CNEN (ultime due colonne).

Questi due ultimi tempi sono stati riportati per mostrare la frequente discordanza, e quindi come ulteriore elemento di giudizio sui criteri interpretativi; va però osservato che essi, a rigore, non sono omogenei con gli altri, in quanto si riferiscono al tempo origine, cioè al tempo valutato all'epicentro e non, come per gli altri, valutato in vari luoghi intorno all'epicentro: ma tale considerazione non basta a giustificare le notevoli differenze che si riscontrano nei dati riportati.

Il confronto dei risultati esposti nelle colonne indicate come colonne II e III dovrebbe servire a riconoscere il criterio con cui il Baratta (o chi per lui) ha svolto la parte per così dire astronomica della conversione, cioè quella relativa all'istante iniziale del tramonto del Sole. In qualche caso il Baratta ha fatto approsimazioni illecite, quale quella [segnalata nell'es. 2 (1687. Aprile 25-26. Amalfi)] di assumere condizioni equinoziali fuori degli equinozi; in altri casi, sono evidenti banali sviste, quale quella [segnalata nell'es. 3 (1730. Maggio 12. Norcia)] di assumere inizi diversi per lo stesso luogo e lo stesso giorno. Si ha comunque una netta impressione di disomogeneità di procedura, al punto che in molti casi non si riesce a riconoscere la procedura medesima.

Il confronto dei risultati esposti nelle colonne indicate come colonne I, IV, V dovrebbe dare una qualche idea sulla natura del tempo in cui il Baratta ha espresso i risultati delle sue conversioni. Il semplice esame delle tre colonne mostra che gli scarti dei tempi dati dal Baratta rispetto ai tempi medi (locali e di Roma) calcolati da noi (in maniera omogenea) sono talmente erratici da non dare direttamente alcuna indicazione utile. Poiché tale fatto potrebbe derivare dalla non omogeneità, dianzi rilevata, dei criteri " astronomici " seguiti dal Baratta, abbiamo normalizzato i dati in questione, calcolando gli scarti dei dati da noi calcolati come se avessimo assunto, di volta in volta, l'istante iniziale preso dal Baratta. 
Orbene, per quanto riguarda i nostri tempi medi locali, l'accordo di essi con i tempi dati dal Baratta, fatta questa normalizzazione, risulta ottimo (scarto nullo) nel $72 \%$ dei 29 casi considerati di ore italiane e buono (scarto non maggiore di $I 5^{\mathrm{m}}$ ) nel $97 \%$ dei casi; queste percentuali scendono al $31 \%$ e al $72 \%$, rispettivamente, se in luogo del tempo medio locale si considera il tempo medio di Roma. Questo sembra indicare che il Baratta tendeva a considerare tempi locali. Resterebbe poi da vedere se questi ultimi fossero tempi medi o tempi veri; tale questione potrebbe avere rilevanza per alcuni dati del 1800 che, nel Baratta, sono espressi con misure al minuto. Al riguardo, abbiamo convertito i dati della colonna IV da tempo medio in tempo vero, ma l'esame dei risultati non ha portato ad alcuna definita opinione al riguardo. Il fatto che in un caso esplicito, e cioé quello illustrato nell'es. 4 (1805, Luglio 26. Molise) il Baratta, partendo da un tempo vero, non abbia sentito il bisogno di convertirlo in tempo medio, fa pensare che egli inclinasse per $\mathrm{i}$ tempi veri: questi ultimi - basta riandare a considerazioni fatte in precedenza - dovevano senz'altro essere la maggioranza nell'epoca considerata, in cui gli orologi venivano di norma controllati mediante meridiane a tempo vero.

La quesione della data. - Come detto prima, nel computo delle ore all'italiana il giorno iniziava all'avemaria della sera, che era dunque l'istante 2400 del giorno che moriva e l'istante 0000 del giorno seguente, con relativo cambio di data.

Per quanto, come detto, approfondite ricerche su attendibili fonti storiche esulassero dal limitato scopo di questo lavoro, abbiamo posto la massima cura nell'esaminare tutte le notizie date nel Baratta e in alcune fonti di più facile consultazione, al fine di formarci una definita opinione al riguardo. Orbene, tale opinione è che effettivamente si usasse cambiare la data del giorno allo spirare delle $24^{\mathrm{h}}$ ital., cioè all'avemaria della sera. Esistevano però usi cronachistici particolari, secondo i quali la notte veniva riferita al giorno immeliatamente precedente, con locuzioni quali "la sera del giomo... », " la notte del... », che potrebbero trarre in inganno. Inoltre, per quanto riguarda il Baratta — e in genere tutti i sismologi della seconda metà del 1800 - 
v'é la consuetudine di "interpretare" le fonti per quanto riguarda l'attribuzione della data; e ciò vien fatto talora correttamente, ma talora non correttamente.

In definitiva, riteniamo che la data indicata nel Baratta e in altri repertori sismologici del passato sia da accettare senza riserve soltanto in casi di cristallina evidenza: precisamente, quando si sia certi della scala di misura (ore temporarie, ore italiane, ore a.m. e p.m.) e, nel caso di ore temporarie e di ore all'italiana, si stia decisamente fuori dell'arco critico fra il tramonto e la mezzanotte. In tutti gli altri casi è invece altamente raccomandabile, per non dire doverosa, la consultazione delle fonti, specialmente di quelle storicamente e geograficamente più vicine all'evento.

A titolo indicativo, abbiamo segnato con un asterisco nella tab. II la data degli eventi " critici " nel senso ora detto $\left({ }^{*}\right)$.

A conforto dell'asserzione secondo la quale la data del giorno, nel computo delle ore all'italiana, cambiasse all'avemaria della sera, riportiamo le seguenti testimonianze, tratte dal Baratta [tra parentesi quadrate, dati o commenti nostri].

1) pag. 95 1541. Ottobre 22-23.

In Alessandria, ad $1^{\text {h }}$ di notte $\left[18^{\mathrm{h}}\right.$ circa] del 22 ottobre si sentì una scossa... Lo Spelta per Pavia [Historia dei Vescovi di Pavia, ca. 1610] dice che alla prima ora del 23 ottobre fu sentito un cosi forte terremoto che fece spaventare tutti.

Come si vede, il cronista usa la data all'italiana, il 23 ottobre, che il Baratta poi correttamente interpreta, in base all'ora, come il 22 ottobre all'uso attuale.

2) pag. 305 1791. Ottobre 12. Dintorni di Monteleone Calabro.

Verso l'Ave Maria (24h ital.) terremoto... A questo terremoto allude certamente il Malvasia-De Rossi (Documenti, ecc.) allorquando dice che nel 13 ottobre in Calabria fu sentita una fortissima scossa...

Anche qui il Baratta mette la data all'uso attuale, mentre gli studiosi citati mettono evidentemente la data delle cronache da loro consultate, cioè la data del giorno 13 , che iniziava all'avemaria (è questo il solo passo

(*) Particolare attenzione quanto al cambiamento di data va posta anche nel passare da tempi locali al tempo universale per eventi accaduti poco dopo la mezzanotte locale. 
del Baratta da cui si tragga esplicitamente che il giorno all'italiana cominciava appunto all'avemaria della sera).

\section{3) pag. 308 1794. Giugno 12. Ariano (Avellino).}

La sera del 12 giugno, verso $3^{h} 20^{m}$ ital. [circa 23h $20^{\mathrm{m}}$ ], in Napoli $e$ nei paesi circonvicini scossa lunga, non leggera...

... nota manoscritta al detto terremoto sentito in Sant'Agata dei Goti: "13 giugno... in questa notte, nell'ora terza e un quarto, si è udito da tutti un fiero terremoto...".

Altro esempio dunque di corretta interpretazione di una cronaca locale con ora data all'italiana.

Un esempio di testimonianza indiretta è il seguente:

4) pag. 111 1599-1600. Cascia (Perugia).

... a $5^{\mathrm{h}}$ della notte [circa $22^{\mathrm{h}} 15^{\mathrm{m}}$ ] del 5 novembre una scossa oltremodo violenta...

Orbene, tuttora nella zona quel terremoto è legato al nome di San Leonardo; questo Santo si festeggia il giorno 6 novembre che a quell'epoca iniziava dunque all'avemaria del 5 .

Un'osservazione simile vale per il terremoto del nursino avvenuto circa alle $19^{\mathrm{h}} 20^{\mathrm{m}}$ del 14 gennaio (all'italiana) 1703 in relazione a San Ponziano, venerato nello spoletino, la cui festa cade appunto il 14 gennaio.

Ecco poi alcune delle accennate locuzioni che attribuiscono la notte al giorno testé spirato.

5) pag. 885 (Bibliografia 1786. Dicembre 25. Rimini.

(Anonimo) Discorso istorico-filosofico sopra il tremuoto che nella notte del di 24, venendo il 25 dicembre dell'anno 1786, dopo le ore 9 $\left[\approx 02^{\mathrm{h}}\right]$ scosse orribilmente la città di Rimini... Cesena 1787.

Ovviamente va letto: "nella notte fra il 24 e il 25 dicembre".

6) pag. 316 1803. Aprile 7. Aquila, Teramo.

Nella notte del 7 aprile, a $5^{h} 1 / 2$ ital. [00 $30^{\mathrm{m}}$ ] in Teramo scossa...

pag. 916 (Bibliografia)

Pannella G., Sul tremuoto avvenuto nella notte seguente del di 7 aprile 1803, Napoli 1888.

Il titolo del saggio sembra indicare la data dell'8 aprile.

7) pag. 318 1805. Luglio 26. Molise.

Fenomeni precursori. - ... fu sentita qualche scossa, fra cui una a $1^{\text {h }}$ della notte $\left[\approx 20^{\mathrm{h}} 50^{\mathrm{m}}\right]$, a $21^{\mathrm{h}}\left[\approx 16^{\mathrm{h}} 50^{\mathrm{m}}\right]$ del giomo $[25]$ e a $9^{\mathrm{h}}\left[\approx 04^{\mathrm{h}}\right.$ $\left.50^{\mathrm{m}}\right]$ della notte antecedente al $26 . A 24^{\mathrm{h}}\left[\approx 19^{\mathrm{h}} 50^{\mathrm{m}}\right]$ di questa giornata gli animali si mostrarono inquieti... [la scossa disastrosa avvenne, come 
detto nel precedente esempio 4) circa alle $22^{\mathrm{h}} 08^{\mathrm{m}}$ tempo medio di Napoli]. pag. 866 (Bibliografia)

Capozzi G., Memoria sul tremuoto avvenuto nel contado di Molise nella sera de' 26 luglio dell'anno 1805, Benevento (s.d.)

pag. 903 (Bibliografia)

(Anonimo) lettera in cui si legge la continuazione e il lucidamento dei danni cagionati in Napoli e nel Regno dall'orribile terremoto accaduto nella notte de' 26 luglio 1805. Roma 1805.

Sulla scorta dei soli elementi qui riportati, sono possibili due interpretazioni. Secondo la prima, la sera e la notte di cui si parla nel titolo del saggio e nella Lettera vanno intese come la prosecuzione del giorno 26, talché quest'ultima è la data dell'evento.

Oppure - e così pensiamo noi - le espressioni vanno prese così cose sono, cioè riferentisi al giorno 26 iniziato alle $24^{\mathrm{h}}$ ital. del giorno 25 , e allora la data giusta è quest'ultima (l'espressione "questa giomata» nella prima citazione si riferirebbe allora al 25 luglio).

Periodo di transizione dalle ore italiane al tempo MEdio DELL'EUROPA CENTRALE.

Natura e attendibilità delle misure. - Come s'è detto, sul finire del 1700 le misure cronometriche in ore iialiane cominciano ad essere sostituite da indicazioni in ore antimeridiane (a.m., ant., a.) e pomeridiane (p.m., pom., p.), contate, le prime da $1^{\mathrm{h}}$ a $12^{\mathrm{h}}$ fra mezzanotte e mezzogiorno, le seconde da $1^{\mathrm{h}}$ a $12^{\mathrm{h}}$ fra mezzogiorno e mezzanotte. Di tale uso,nato nei paesi tedesci e diffusosi poi in Francia e negli altri paesi europei già nei secoli $16^{\circ}-17^{\circ}$, v'é, nel Baratta e in altri repertori sismologici del passato, qualche esempio relativo a località dell'Italia settentrionale ( ${ }^{*}$ ) anche prima dell'epoca (appunto il finire del 1700) in cui l'occupazione da parte degli eserciti francesi lo impose in tutta l'Italia.

$E^{\prime}$ da osservare che tali indicazioni furono inizialmente (come quelle in ore italiane) in tempo solare vero, ovviamente locale. Ripetendo cose già dette sopra, ricorderemo che successivamente

(*) Specialmente località del Piemonte e della Liguria, in cui l'influenza francese fu sempre molto forte: per esempio il Mercalli (ripreso poi dal Baratta) ne "I terremoti della Liguria e del Piemonte" (Napoli 1897) cita fonti con indicazioni in ore a.m. e p.m. già nel secolo $17^{\circ}$. 
le misure orarie furono in tempo medio locale (grosso modo, e sporadicamente, a partire dal $1810 \div 20$ ); poi, facendo forza sugli orologi degli uffici telegrafici e delle stazioni ferroviarie, furono espresse in tempo medio di Roma, all'incirca dal 1866; infine, dal $1^{\circ}$ gennaio 1894 (a seguito del citato R.D. del novembre 1893), in tempo medio dell'Europa centrale, che è il tempo di cui ancora oggi ci si serve in Italia.

Il periodo tra la fine del 1700 e la fine del 1800 è un periodo di grande confusione anche per i dati orari riferentisi a eventi sismici. Per questi ultimi, la situazione si fa chiara, almeno per i dati "ufficiali ", soltanto dal $1^{\circ}$ gennaio 1894 , data a partire dalla quale - come come già accennato - il "Bollettino della Società Sismologica Italiana " (e con esso tutti gli altri periodici scientifici italiani che riportavano notizie sismologiche) adottò il tempo medio dell'Europa centrale. Precedentemente, in mancanza di indicazioni esplicite o di illuminanti notizie collaterali, non si sa, quando si scorrono repertori sismologici di quel periodo, se si ha a che fare con ore di tempo medio di Roma o di tempo medio locale o di tempo vero locale, se non addirittura ancora di ore italiane: lo stesso Baratta in qualche caso $\left({ }^{*}\right)$ indica l'impossibilità di interpretare le misure che sono riportate nelle fonti.

Cosi, mentre per il precedente periodo delle ore italiane l'attenzione va riportata essenzialmente sulla correttezza del calcolo per la conversione in tempo medio o in tempo universale e per l'attribuzione della data, per molti eventi di questo periodo di transizione l'attenzione va riportata principalmente sulla corretta scelta della scala di tempo in cui vanno lette le misure.

L'opinione che ci siamo formata esaminando alcuni casi notevoli è che in genere le misure indicate nelle fonti come ore a.m. o p.m. senza uleriore specificazione siano da prendere come ore di tempo vero locale sino al 1860-70, dopodiché (fino al 1894) possano essere interpretate come ore di tempo medio, in qualche caso di tempo medio di Roma se provenienti da grandi centri abitati serviti dalla ferrovia e dotati di uffici telegrafici principali.

$\left(^{*}\right)$ Per esempio, pag. 395 (1845, settembre 13. Lucca): «A $1^{\text {th }} 15^{m}$ (ant. o pom. od ital.?) una violenta ma breve scossa...". 
Ma è un'opinione che va presa come criterio di larghissima massima, data la notevole complessità della situazione allora esistente.

Per dar conto di questa complessità, e anche per introdurre il discorso sulla precisione da attribuire alle misure in discussione, richiamiamo l'App. C., che contiene passi di carattere cronometrico tratti da uno studio particolareggiato di A. Serpieri sul terremoto riminese del 18 marzo 1875, dei quali ci siamo già serviti, per scopi analoghi, nella discussione sull'attendibilità delle misure in ore italiane.

Quei passi sono abbastanza indicativi sulla persistente pochezza di gran parte delle misurazioni cronometriche in un'epoca in cui la sismologia era già entrata nella fase "strumentale". V'é tuttavia da rilevare un sostanziale progresso rispetto al passato: infatti, grazie alla maggiore e più rapida circolazione delle notizie mediante il servizio telegrafico e quello postale per ferrovia, si era in grado di avere piuttosto presto informazioni sulla qualità degli orologi adoperati, e ciò consentiva di fare attendibili stime sulla precisione delle misure.

Genericamente parlando, l'errore assoluto delle misure di questo periodo può essere stimato dell'ordine da qualche secondo a $20 \div 30$ secondi se le misure erano date in ore, minuti, secondi, il che accadeva quando la sorgente era l'orologio di un Osservatorio (*); di $1 \div 2$ minuti per le misure date al minuto o fra. zione di minuto, che fanno presumere, come sorgente, un buon orologio meccanico; di $t 5^{\mathrm{m}}$ per le misure arrotondate ai $5^{\mathrm{m}}$ e $\mathrm{di} \pm 10^{\mathrm{m}}$ per quelle arrotondate al quarto d'ora che fanno pensare, come sorgenti, a orologi civici.

Tornando all'App. C., è da osservare che il Serpieri fa un accurato elenco dei dati orari riguardanti il terremoto in esame con ampie notizie collaterali (alcune delle quali costituiscono appunto la materia dell'Appenlice medesima): va rilevato che

(*) Accadeva talora che uno o più degli orologi a pendolo di un Osservatorio si fermassero a causa della scossa sismica. La prima misura "al secondo " riportata dal Baratta $\left(10^{\mathrm{h}} 1 \mathrm{~m} 40^{\mathrm{s}}\right.$ t.v., terremoto del Molise. 26 luglio 1805, discussa nel precedente es, 4) di conversione di ore italiane) riguarda appunto la "fermata " di un pendolo astronomico. Altre 
solo in minima parte le misure sono in tempo medio di Roma (che allora peraltro era già il tempo "nazionale") mentre predominano tempi medi e veri locali. L'autore riduce tutti questi vari tempi in tempo medio di Roma e, dopo un'ampia analisi, assegna allo scossa principale l'istante $00^{\mathrm{h}} 52^{\mathrm{m}} 44^{\mathrm{s}}$ t.m. di Roma. Il Baratta (pag. 466) dice: "La scossa, avvenuta verso le $0^{\prime \prime} 51^{m}$ ant. del 18 marzo...", senza specificare che si tratta di tempo medio di Roma; questo fatto fa pensare che, almeno per eventi del periodo intorno al 1888 (anno in cui fu pubblicato lo studio del Serpieri (*), il Baratta usasse di norma il tempo medio di Roma. E' molto probabile che tale periodo possa essere fatto partire almeno dal 1872, da quando cioè presero ad essere pubblicati, a cura di M.S. De Rossi, regolari bollettini sismici, presto inseriti nel "Bullettino del Vulcanismo Italiano" (dal 1873), nei quali, come avverte un'apposita nota, le ore sono espresse "per lo più in tempo medio di Roma"("*). Siamo pure inclini a pensare che in tale tempo medio siano espressi gli istanti che compaiono in studi di sintesi fatti nel periodo 1866-1894 (come, per es., molti studi particolari del Baratta). Esclusi questi casi, e cioè per indicazioni originali, c'è da pensare piuttosto, come detto poco sopra, che si tratti di tempo medio locale se la notizia

misure in secondi si hanno nel 1851 da Milano (terremoto Lombardo-Ticinese del 5 febbraio) e da Napoli (terremoto del Vulture, del 14 agosto); poi, dal 1860, più frequentemente (ma sempre da osservatori astronomici e poi anche da osservatori sismologici), talora con l'indicazione dell'errore assoluto (terremoto dei Colli Laziali del 22 gennaio $1892: 11^{\mathrm{h}} 25^{\mathrm{m}} 12^{\mathrm{s}} \pm 6^{\mathrm{s}}$ pom.); va notato tuttavia che questo terremoto è stato oggetto di uno studio particolareggiato.

(*) La pubblicazione avvenne nel 1889 a cura di G. Giovannozzi.

$(* *)$ Il Baratta, in una nota a pag. 456 , avverte che la pubblicazione del "Bullettino del Vulcanismo Italiano" gli consentirà da allora in poi (siamo al gennaio 1873) di essere più succinto nella descrizione degli eventi e in particolare di omettere la serie cronologica delle repliche. Ciò significa adesione ai criteri cronometrici adottati nel "Bullettino ", cioè l'adozione del tempo medio di Roma. E' da osservare che il Baratta parla esplicitamente di tempo medio di Roma soltanto in tre occasioni: la prima, relativamente al terremoto garganico dell'8 dicembre 1889 (per il quale riporta i dati orari di un suo studio), e le altre due per terremoti greci, sentiti in Italia (Candia, 17 luglio 1887; Epiro, 14 giugno 1893). 
viene da una città e molto probabilmente di tempo vero se la notizia vien da un piccolo centro. Ancora una volta si rivela determinante la consultazione degli studi originali e/o delle fonti storiche.

Conversione al tempo universale. - Nel caso in cui le misure orarie afferenti ad un medesimo evento siano date parte in tempo vero, parte in tempo medio di Roma (come nell'evento di cui all'App. C), può risultare vantaggioso, anche per confrontarsi con studi dell'epoca, adottare una procedura di riduzione progressiva, dal tempo vero al tempo medio locale, da quest'ultimo al tempo medio di Roma, e infine al tempo universale: tale è appunto la procedura qui descritta,anche se nulla vieta ( e anzi in qualche caso può risultare conveniente) di effettuare conversioni dirette in $\mathrm{TU}$, secondo le [15] $\div$ [18].

Il primo passo, comune a tutti i casi è di esprimere le misure in notazione a $24^{\mathrm{h}}$, anziché duodecimale; ciò si fa, com'é ben noto, lasciando inalterate le misure in ore antimeridiane e aggiungendo $12^{\mathrm{h}}$ a quelle in ore pomeridiane $\left(^{*}\right)$.

a) Conversione del tempo vero in tempo medio locale:

si attua togliendo dal tempo vero $\left(^{* *}\right)$ l'equazione del tempo $E$ (con il suo segno), secondo la [8] (vedi App. E).

b) Conversione del tempo medio locale in tempo medio di Roma:

si ottiene, socendo la [9], aggiungendo al primo la longitudine $\left(\lambda_{A}\right)_{R}$ del luogo rispetto a Roma (in ore),

$$
\left(t_{m}\right)_{R}=\left(t_{m}\right)_{A}+\left(\lambda_{A}\right)_{R}
$$

(*) E' sorprendente che questa semplice operazione abbia portato a errori grossolani anche in epoca recente; prima di noi, già altri hanno rilevato tale sconcertante fatto: per es., G. Agamennone [Boll. Soc. Sism. It., VI (1900-01), pag. 45] e A. Cavasino [ibidem, XX (1916) pag. 14].

(**) Ricordiamo che $\mathrm{i}$ tempi veri e medi qui nominati partono dalla mezzanotte anziché dal mezzogiorno. 
presa positiva oppure negativa a seconda che il luogo si trovi a ovest oppure a est rispetto a Roma. Nell'App. D sono riportate le longitudini rispetto a Roma (sia in ore, sia in minuti) delle città capoluogo di provincia.

c) Conversione del tempo di Roma in tempo universale:

si effettua, ancora secondo la [9], togliendo la longitudine di Roma (che per l'epoca considerata è la longitudine dell'Osservatorio del Collegio romano) rispetto a Greenwich (espressa in tempo) $\left({ }^{*}\right)$,

$$
\mathrm{TU}=\left(t_{m}\right)_{R}-0,832.04^{\mathrm{h}}=\left(t_{m}\right)_{\mathrm{R}}-49^{\mathrm{m}} 55,55^{\mathrm{s}} \approx\left(t_{m}\right)_{R}-50^{\mathrm{m}}
$$

Vogliamo attirare l'attenzione sul fatto che nel periodo immediamente precedente l'adozione del tempo medio dell'Europa centrale, e cioè all'incirca dal 1872 al 1893 compreso, $i$ dati orari nei citati "Bollettini » sismologici, come pure negli studi particolari, sono esplicitamente espressi per lo piu in tempo medio di Roma, talché occorre sottrarre da essi, per passare al tempo uni versale, $\approx 50$ minuti, e non un'ora esatta, come, invece, deve operare per le misure in T.M.E.C. Si ha l'impressione che non sempre si sia tenuta presente questa circostanza.

Periodo Successivo AL 1894.

In questo periodo non dovrebbero più esistere praticamente problemi di interpretazione di misure orarie in quanto queste sono date in tempo del fuso orario $-1^{\text {h }}\left(15^{\circ} \mathrm{E}\right)$.

Per il passaggio al tempo universale basta semplicemente togliere un'ora esatta. Questioni di carattere generale consistono nel porre la dovuta attenzione - come più volte segnalato all'eventuale cambiamento della data nel passare al tempo universale per eventi iniziati entro circa un'ora dopo la mezzanotte

(*) Cfr. tab. 1 a pag. 164. 
e, inoltre nel fare attenzione ai periodi di vigenza dell'ora estiva e all'inserzione di secondi intercalari di tempo universale coordinato.

Resta tuttavia da fare qualche osservazione relativamente alla precisione delle misure.

A tale riguardo, occorre fare una netta distinzione fra il periodo che arriva fino a circa il 1930 e il periodo successivo, nel quale ultimo ci si è potuti giovare di radiosegnali orari (trasmessi, in un primo momento, da stazioni di radiodiffusione e, successivamente, anche da apposite stazioni radiotrasmittenti).

Anche nel primo di tali periodi la sismologia è diventata ormai decisamente "strumentale" $\left({ }^{*}\right)$, nel senso che quasi tutte le osservazioni vengono fatte mediante strumenti dotati di registratori grafici o fotografici il cui asse dei tempi è comandato e/o controllato da orologi meccanici; continui perfezionamenti vengono apportati agli strumenti sia per quanto riguarda l'uniformità di marcia della zona di registrazione sia per quanto riguarda la qualità degli orologi di controllo. Se però si scorrono i bollettini sismici dei primi decenni del presente secolo si resta sorpresi nel constatare la cattiva qualità media delle misure orarie (salvo quelle provenienti da alcuni Osservatori particolarmente ben equipaggiati e ben gestiti), della quale un significativo indice è la frequente discordanza di misure relative a uno stesso evento provenienti da località vicine fra loro, talora vicinissime. Se in qualche caso ciò è imputabile alla scadente qualità degli orologi di controllo e dei movimenti di orologeria dei registratori (nei bollettini, le notizie di guasti a questi dispositivi sono piuttosto frequenti), nella maggior parte dei casi la causa va ricercata nella difficoltà di ben regolare gli orologi.

In alcune grandi città tale regolazione era resa possibile da appositi segnali ottici emessi o controllati dal locale Osservatorio astronomico che indicavano l'istante del mezzogiorno $\left({ }^{* *}\right)$;

(*) Qualche riserva ancora andrebbe fatta per gli eventi segnalati soltanto macrosismicamente, cioè per i quali manchino notizie strumentali.

$\left.{ }^{* *}\right)$ A Roma, per es. - ma usi simili si seguirono anche in altre grandi città -, il mezzogiorno era indicato da un colpo di cannone e dall'abbassarsi di un pallone colorato di rosso issato sulla torre dell'Osservatorio 
nelle città sprovviste di questo servizio ma provviste di Osservatorio astronomico la regolazione era possibile riferendosi agli orologi dell'Osservatorio: per es. portando in questo ultimo un buon cronometro da tasca regolando questo orologio e poi tornati all'Osservatorio sismologico, regolando sul cronometro gli altri orologi. Ma nelle città sprovviste di specola e, in generale, nei centri medi e piccoli, a meno di attrezzarsi localmente per il controllo astronomico del tempo, aggiungendo qualcosa di meglio alla vecchia meridiana, non v'era che fidarsi dei propri orologi e del controllo con gli orologi del telegrafo e della ferrovia. Per questi motivi una particolare attenzione va portata alla valutazione dell'errore delle misure e quindi del peso da attribuire a ciascuna di esse in sede di confronto reciproco.

Fortunatamente, il progressivo aumento degli Osservatori sismologici adeguatamente attrezzati, ha reso via via meno importanti le misure orarie attinte da altre fonti. In prosieguo di tempo, la situazione è ulteriormente migliorata in quanto l'accennata diffusione di radiosegnali orari ha consentito ovunque il controllo locale degli orologi con cadenza almeno giornaliera (all'inizio, al decimo di secondo, poi entro qualche centesimo di secondo); l'adozione di orologi piezoelettrici, capaci di conser vare con grande costanza il tempo fra un controllo con radiose. gnali orari e il successivo, e di registratori ad alta velocità di scorrimento (fra 0,5 e $2 \mathrm{~mm} / \mathrm{s}$ per quelli grafici normali; anche più alta per registratori grafici speciali e per quelli magnetici) ha poi notevolmente migliorato la precisione delle misure che, da un valore medio stimato di circa $+5^{\text {s }}$ all'inizio del secolo, può considerarsi oggi mediamente dell'ordine di +0.2 operando con buoni registratori grafici.

del Collegio romano. Una lapide murata vicino alla meridiana esistente nella chiesa di S. Maria degli Angeli ricorda che la meridiana medesima "inaugurata il 6-10-1702 servì a regolare gli orologi di Roma fino al 1846 quando il cannone cominciò ad annunciare il mezzodì ». Quest'ultimo uso - per sua natura non molto preciso (come tutti i segnali acustici) per regolare orologi in luoghi appena appena un po' distanti - è andato avanti fino a qualche anno fa. Il segnale ottico dall'Osservatorio del Collegio romano fu poi portato, quando quell'Osservatorio si fuse con l'Osservatorio del Campidoglio, sulla torre capitolina, e consistette nello spegnimento, a mezzogiorno preciso, di lampade elettriche di colore rosso. 
Già a questi livelli di precisione ( e ancor più con quelli, più elevati, raggiungibili con registratori magnetici e particolari tecniche di registrazione) cominciano a diventare non trascurabili alcune cause di errore che potremmo chiamare del secondo ordine rispetto a quelle, ben più rilevanti, esistenti qualche tempo fa; per es., in ordine alla lettura delle registrazioni, l'errore introdotto da deformazioni della zona di registrazione per sollecitazioni meccaniche o trattamenti fisico-chimici da essa subiti e, in ordine alla precisione intrinseca delle marche di tempo, e quindi alla contemporaneità di marche corrispondentisi in registrazioni fatte in luoghi diversi, l'errore introdotto dalla diversità e dalle eventuali variazioni del tempo di propagazione dei segnali orari e di sincronizzazione.

Ma queste, come ben si comprende, sono questioni da affrontare in un contesto diverso da quello in cui è stato sviluppato il presente lavoro. 
APPENDice A.

SECONDI INTERCALARI DI TEMPO TUC

Alla data del 1 gennaio 1972 sono stati inseriti $+10^{\text {s }}$ Alla data del 1 luglio 1972 sono stati inseriti $+1^{\text {s }}$ Alla data del 1 gennaio 1973 sono stati inseriti $+1^{\text {s }}$ Alla data del 1 gennaio 1974 sono stati inseriti $+1^{\text {s }}$ Alla data del 1 gennaio 1975 sono stati inseriti $+1^{\text {s }}$ Alla data del 1 gennaio 1976 sono stati inseriti $+1^{\text {s }}$ Alla data del 1 gennaio 1977 sono stati inseriti $+1^{\text {s }}$ Alla data del 1 gennaio 1978 sono stati inseriti $+1^{\text {s }}$ Alla data del 1 gennaio 1979 sono stati inseriti + 1s $^{\text {s }}$ 
APPENDice B

Periodi di Vigenza dell'ora legale (estiva) in Italia

\begin{tabular}{|lrlr|llll} 
Da ore 24 di sab. & 3 giugno & 1916 & a ore 24 di sab. 30 settembre 1916 \\
Da ore 24 di sab. & 31 & marzo & 1917 & a ore 24 di dom. 30 settembre 1917 \\
Da ore 24 di sab. & 9 marzo & 1918 & a ore 24 di dom. 6 ottobre & 1918 \\
Da ore 24 di sab. & 1 marzo & 1919 & a ore 24 di sab. 4 ottobre & 1919 \\
Da ore 24 di sab. 20 marzo & 1920 & a ore 24 di sab. 18 settembre 1920
\end{tabular}

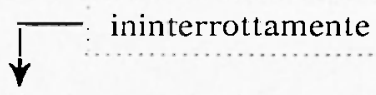

Da ore 24 di ven. 14 giugno $1940 \mid$ a ore 03 di lun. 2 novembre 1942 Da ore 02 di lun. 29 marzo 1943 a ore 03 di lun. 4 ottobre 1943 Da ore 02 di dom. 2 aprile 1944 a ore 03 di dom. 17 settembre 1944 Da ore 02 di lun. 2 aprile 1945 a ore 24 di sab. 15 settembre 1945 Da ore $02 \mathrm{di}$ dom. 17 marzo 1946 a ore 02 di dom. 6 ottobre 1946 Da ore 00 di dom. 16 marzo 1947 a ore 01 di dom. 5 ottobre 1947 Da ore 02 di dom. 29 febbr. 1948 a ore 03 di dom. 3 ottobre 1948 Da ore 00 di dom. 22 maggio 1966 a ore 24 di sab. 24 settembre 1966 Da ore 00 di dom. 28 maggio 1967 a ore 01 di dom. 24 settembre 1967 Da ore 00 di dom. 26 maggio 1968 a ore 01 di dom. 22 settembre 1968 Da ore 00 di dom. 1 giugno 1969 a ore 01 di dom. 28 settembre 1969 Da ore $00 \mathrm{di}$ dom. 31 maggio 1970 a ore 01 di dom. 27 settembre 1970 Da ore 00 di dom. 23 maggio 1971 a ore 01 di dom. 26 settembre 1971 Da ore 00 di dom. 28 maggio 1972 a ore 01 di dom. 1 ottobre 1972 Da ore 00 di dom. 3 giugno 1973 a ore 01 di dom. 30 settembre 1973 Da ore 00 di dom. 26 maggio 1974 a ore 01 di dom. 29 settembre 1974 Da ore 00 di dom. 1 giugno 1975 a ore 01 di dom. 28 settembre 1975 Da ore 00 di dom. 30 maggio 1976 a ore 01 di dom. 26 settembre 1976 Da ore $00 \mathrm{di}$ dom. 22 maggio 1977 a ore 01 di dom. 25 settembre 1977 Da ore 00 di dom. 28 maggio 1978 a ore 01 di dom. 1 ottobre 1978 Da ore 00 di dom. 27 maggio 1979 a ore 01 di dom. 30 settembre 1979 
Appendice C

\section{PASSI CON NOTAZIONI CRONOMETRICHE ESTRATTI DA:}

Alessandro SERPIERI, "Scritti di sismologia", nuovamente raccolti e pubblicati da G. Giovannozzi. Parte II. I terremoti del 18 marzo 1875 $e$ del 28 luglio 1883 ". Firenze $1889\left({ }^{*}\right)$.

Acqualagna Ora data: 12.47 tempo vero

"In quanto all'ora debbo dirle che l'orologio pubblico non va mai bene. Io però ho un discreto cilindro, che vado rimettendo con una meridiana ".

Bagno

Ora data: 12.45 tempo vero

"Batteva la mezzanotte e tre quarti al nostro orologio comunale, quando cominciò a sentirsi il terremoto..."

Cagli

Ora data: 12.49 tempo vero

«Gli orologi privati dettero 12.48 ovvero 12.50 . Ho preso la media: non ho potuto sapere quanto fosse l'accordo di quegli orologi con la meridiana, e quanto la meridiana sia esatta ".

Cagli [altro osservatore]

Ora data: 12.45 t.m. di Roma

«... l'orologio ... rimesso alla meridiana due giorni prima, e che d'ordinario in due o tre giorni non suol far variazione".

Camerino

Ora data: 12.55 tempo medio locale

«L'orologio del sismometro scattó, e paragonato con altro a t.m. locale, verificato con un regolatore, indicò che nel momento del terremoto erano le 12.55 t.m. locale ».

Cesena

Ora data: 12.56 t.m. di Roma

Il professore Francavilla ebbe l'ora dal capo della stazione della ferrovia, il quale la ottenne da un suo orologio privato che si fermò per la scossa.

Iesi

Ora data: 12.56 t.m. di Roma

"L'ora da me data è quella della stazione ferrovaria di Iesi. L'orologio della ferrovia ogni giorno viene regolato a dovere col t.m. di Roma".

(*) [L'autore riporta, da pag. 53 a pag. 134, osservazioni fatte in vari Csservatori e luoghi, con commenti suoi o degli osservatori, questi ultimi fra virgolette; le ore sono tutte p.m.; fra parentesi quadrate sono dati i commenti nostri]. 
segue APP. C

Mantova

Ora data: 12.58 o 12.59 t.m. di Roma

«L'orologio sonò l'un'ora ant. appena uno o due minuti dopo la scossa".

[Il Serpieri intepreta questa informazione come riferentesi al t.m. di Roma].

Modigliana

Ora data: 12.44 t.v.

"Riguardo all'ora, il temperatore dell'orologio mi disse di non averlo rimesso nè il 17 nè il 18, perché era esatto con l'ombra del gnomone e col cronometro ».

Moncalieri

Ora data: 1.25 t.m. di Nizza

«...In tutta la Francia ...ogni città segue il suo tempo locale, mentre gli orologi delle stazioni ferroviarie segnano invece l'ora di Parigi ». [In Italia, e anzi in tutta l'Europa, la situazione era analoga].

\section{Perugia}

Ora data: 12.55 t.m. di Roma

«L'ora è approssimativa. Il telegrafo era chiuso, e alla ferrovia non si badò al fenomeno... I confronti fatti poi della maggior parte degli orologi con quello del telegrafo porterebbero ore 12.55. Altri insistono per ore 12.53; ma io direi di stare alla prima ».

\section{Ravenna \\ Ora data: 12,55 t.m. di Roma}

"L'orologio della stazone, osservato subito dopo dall'uomo di guardia, dette l'ora ...".

\section{Rimini}

"Gli orologi della stazione si sono fermati a quest'ora "...Altri ancora hanno scritto che si fermò l'orologio della piazza della Fontana a 12.54, e che la sera era stato regolato col tempo della ferrovia. Ma poiché da un mezzodi all'altro avanzava in quei giorni di $2^{\mathrm{m}} 30^{\mathrm{s}}$, si potrebbe supporre che l'ora sua corretta fosse $12.551 / 2$.

\section{Udine}

Ora data: $1^{\text {h }}$ tempo medio locale

“... nell'istante in cui l'orologio civico batteva il tocco. Detto orologio è regolato a tempo medio del luogo".

\section{Urbino}

Ora data: $12.51 \quad 1 / 2$ tempo medio di Roma

"...Mi sono portato all'ufficio telegrafico, e dopo fatte le debite correzioni [probabilmente perché l'orologio dell'osservatore era regolato a tempo medio. locale] ho scelta l'ora media fra l'osservazione del telegrafista e la mia. (Io avevo ore $12.50 \circ$ al più 12.51; il telegrafista dava $12.512 / 3 »$. 
APPENDice D

COORDINATE GEORAFICHE DEI CAPOLUOGHI DI PROVINCIA $\left({ }^{*}\right)$

\begin{tabular}{|c|c|c|c|c|c|}
\hline \multirow{2}{*}{$\begin{array}{c}\text { CAPOLUOGHI } \\
\text { DI } \\
\text { PROVINCIA }\end{array}$} & \multirow{2}{*}{$\begin{array}{c}\text { lat. } N \\
\varphi \\
\text { (gradi) }\end{array}$} & \multicolumn{2}{|c|}{$\begin{array}{c}\text { longitudine } \lambda_{\mathrm{E}} \\
\text { Greenwich }\end{array}$} & \multicolumn{2}{|c|}{$\begin{array}{l}\text { longitudine } \lambda \\
\text { da Roma }\left({ }^{\star \star}\right)\end{array}$} \\
\hline & & (in ore) & (in $\min )$. & (in ore) & (in $\min$.) \\
\hline Agrigento & 37,309 & 0,9057 & 54,34 & $-0,0736$ & $-4,42$ \\
\hline Alessandria & 44,914 & 0,5739 & 34,43 & $+0,2582$ & $+15,49$ \\
\hline Ancona & 43.613 & 0,9017 & 54,10 & $-0,0696$ & $-4,18$ \\
\hline Aosta & 45,739 & 0,4875 & 29,25 & $+0,3445$ & $+20,67$ \\
\hline Arezzo & 43,459 & 0,7921 & 47,53 & $+0,0399$ & $+2,39$ \\
\hline Ascoli Piceno & 42,856 & 0,9053 & 54,32 & $-0,0732$ & $-4,39$ \\
\hline Asti & 44,896 & 0,5468 & 32,81 & $+0,2852$ & $+17,11$ \\
\hline Avellino & 40,910 & 0,9859 & 59,16 & $-0,1539$ & $-9,23$ \\
\hline Bari & 41,126 & 1,1242 & 67,45 & $-0,2922$ & $-17,53$ \\
\hline Belluno & 46,135 & 0,8145 & 48,87 & $+0,0175$ & $+1,05$ \\
\hline Benevento & 41,126 & 0,9845 & 59,07 & $-0,1525$ & $-9,15$ \\
\hline Bergamo & 45,694 & 0,6442 & 38,65 & $+0,1878$ & $+11,27$ \\
\hline Bologna & 44,493 & 0,7559 & 45,35 & $+0,0762$ & $+4,57$ \\
\hline Bolzano & 46.505 & 0,7569 & 45,42 & $+0,0751$ & $+4,51$ \\
\hline Brescia & 45,541 & 0,6811 & i), 87 & $+0,1509$ & $+9,05$ \\
\hline Brindisi & 40,635 & 1,1957 & 71,74 & $-0,3637$ & $-21,78$ \\
\hline Cagliari & 39,221 & 0,6073 & 36,44 & $+0,2247$ & $+13,48$ \\
\hline Caltanisetta & 37,491 & 0,9371 & 56,23 & $-0,1051$ & $-6,31$ \\
\hline Campobasso & 41,554 & 0,9770 & 58,62 & $-0,1450$ & $-8,70$ \\
\hline Caserta & 41,068 & 0,9556 & 57,34 & $-0,1236$ & $-7,42$ \\
\hline Catania & 37,500 & 1,0055 & 60,33 & $-0,1735$ & $-10,41$ \\
\hline Catanzaro & 38,901 & 1,1061 & 66,37 & $-0,2741$ & $-16,45$ \\
\hline
\end{tabular}

${ }^{*}$ Approssimativamente, il centro della città.

${ }^{* *}$ Riferita all'origine del tempo medio di Roma, e cioè all'Osservatorio del Collegio romano $\left(0,8321^{\mathrm{h}}\right.$ E. Greenwich).

Il segno - vale per le longitudini a Est del meridiano di Roma.

Il segno + vale per le longitudini a Ovest del meridiano di Roma. N.B. - Tutte le città elencate sono a Est del meridiano Greenwich. 
segue APP. D

COORIJNATE GEORAFICHE DEI CAPOLUOGH DI PROVINCIA

\begin{tabular}{|c|c|c|c|c|c|}
\hline \multirow{2}{*}{$\begin{array}{c}\text { CAPOLUOGHI } \\
\text { DI } \\
\text { PROVINCIA }\end{array}$} & \multirow{2}{*}{$\begin{array}{c}\text { lat. } N \\
\varphi \\
\text { (gradi) }\end{array}$} & \multicolumn{2}{|c|}{$\begin{array}{c}\text { longitudine } \lambda_{E} \\
\text { Greenwich }\end{array}$} & \multicolumn{2}{|c|}{$\begin{array}{l}\text { longitudine } \lambda \\
\text { da Roma }\left(^{* *}\right)\end{array}$} \\
\hline & & (in ore) & (in $\min$.) & (in ore) & (in $\min$.) \\
\hline Chieti & 42,337 & 0,9451 & 56,70 & $-0,1130$ & $-6,78$ \\
\hline Como & 45,802 & 0,6051 & 36,31 & $+0,2269$ & $+13,61$ \\
\hline Cosenza & 39,293 & 1,0833 & 65,00 & $-0,2512$ & $-15,07$ \\
\hline Cremona & 45,135 & 0,6682 & 40,09 & $+0,1638$ & $+9,83$ \\
\hline Cuneo & 44,392 & 0,5029 & 30,18 & $+0,3291$ & $+19,75$ \\
\hline Enna & 37,563 & 0,9517 & 57,10 & $-0,1187$ & $-7,12$ \\
\hline Ferrara & 44,838 & 0,7750 & 46,50 & $+0,0570$ & $+3,42$ \\
\hline Firenze & 43,770 & 0,7497 & 44,98 & $+0,0823$ & $+4,94$ \\
\hline Foggia & 41,459 & 1,0365 & 62,19 & $-0,2045$ & $-12,27$ \\
\hline Forlì & 44,198 & 0,8025 & 48,15 & $+0,0295$ & $+1,77$ \\
\hline Frosinone & 41,640 & 0,8901 & 53,41 & $-0,0581$ & $-3,49$ \\
\hline Genova & 44,414 & 0,5957 & 35,74 & $+0,2364$ & $+14,18$ \\
\hline Gorizia & 45,944 & 0,9077 & 54,46 & $-0,0756$ & $-4,54$ \\
\hline Grosseto & 42,766 & 0,7409 & 44,45 & $+0,0912$ & $+5,47$ \\
\hline Imperia & 43,885 & 0,5361 & 32,16 & $+0,2960$ & $+17,76$ \\
\hline Isernia & 41,590 & 0,9481 & 56,88 & $-0,1160$ & $-6,96$ \\
\hline L'Aquila & 42,347 & 0,8931 & 53,58 & $-0,0610$ & $-3,66$ \\
\hline La Spezia & 44,108 & 0,6550 & 39,30 & $+0,1770$ & $+10,62$ \\
\hline Latina & 41,466 & 0,8603 & 51,62 & $-0,0283$ & $-1,70$ \\
\hline Lecce & 40,356 & 1,2116 & 72,70 & $-0,3796$ & $-22,77$ \\
\hline Livorno & 43,550 & 0,6873 & 41,24 & $+0,1448$ & $+8,69$ \\
\hline Lucca & 43,842 & 0,7000 & 42,00 & $+0,1320$ & $+7,92$ \\
\hline Macerata & 43,294 & 0,8967 & 53,80 & $-0,0647$ & $-3,88$ \\
\hline Mantova & 45,155 & 0,7196 & 43,18 & $+0,1124$ & $+6,75$ \\
\hline Massa & 44.036 & 0,6763 & 40,58 & $+0,1558$ & $+9,35$ \\
\hline Matera & 40,667 & 1,1073 & 66,44 & $-0,2752$ & $-16,51$ \\
\hline Messina & 38,185 & 1,0366 & 62,20 & $-0,2046$ & $-12,27$ \\
\hline
\end{tabular}


segue Apr. D

COORDINATE GEORAFICHE DEI CAPOLLOGII DI PROVINCIA

\begin{tabular}{|c|c|c|c|c|c|}
\hline \multirow{2}{*}{$\begin{array}{c}\text { CAPOLUOGHI } \\
\text { DI } \\
\text { PROVINCIA }\end{array}$} & \multirow{2}{*}{$\begin{array}{c}\text { lat. } N \\
\varphi \\
\text { (gradi) }\end{array}$} & \multicolumn{2}{|c|}{$\begin{array}{c}\text { longitudine } \lambda_{\mathrm{E}} \\
\text { Greenwich }\end{array}$} & \multicolumn{2}{|c|}{$\begin{array}{l}\text { longitudine } \lambda \\
\text { da Roma }\left(^{* *}\right)\end{array}$} \\
\hline & & (in ore) & (in min.) & (in ore) & (in min.) \\
\hline Milano & 45,464 & 0,6121 & 36,73 & $+0,2199$ & $+13,20$ \\
\hline Modena & 44,646 & 0,7284 & 43,70 & $+0,1036$ & $+6,22$ \\
\hline Napoli & 40,856 & 0,9510 & 57,06 & $-0,1190$ & $-7,14$ \\
\hline Novara & 45,446 & 0,5739 & 34,44 & $+0,2581$ & $+15,49$ \\
\hline Nuoro & 40,315 & 0,6220 & 37,32 & $+0,2100$ & $+12,60$ \\
\hline Oristano & 39,901 & 0,5725 & 34,35 & $+0,2596$ & $+15,57$ \\
\hline Padova & 45,414 & 0,7913 & 47,48 & $+0,0407$ & $+2,44$ \\
\hline Palermo & 38,113 & 0,8918 & 53,51 & $-0,0598$ & $-3,59$ \\
\hline Parma & 44,802 & 0,6883 & 41,30 & $+0,1437$ & $+8,62$ \\
\hline Pavia & 45,185 & 0,6098 & 36,59 & $+0,2222$ & $+13,33$ \\
\hline Perugia & 43,108 & 0,8259 & 49,56 & $+0,0061$ & $+0,37$ \\
\hline Pesaro & 43,914 & 0,8609 & 51,66 & $-0,0289$ & $-1,73$ \\
\hline Pescara & 42,459 & 0,9475 & 56,85 & $-0,1155$ & $-6,93$ \\
\hline Piacenza & 45,050 & 0,6461 & 38,77 & $+0,1859$ & $+11,16$ \\
\hline Pisa & 43,712 & 0,6930 & 41,58 & $+0,1390$ & $+8,34$ \\
\hline Pistoia & 43,928 & 0,7275 & 43,65 & $+0,1045$ & $+6,27$ \\
\hline Pordenone & 45,958 & 0,8435 & 50,61 & $-0,0115$ & $-0,69$ \\
\hline Potenza & 40,637 & 1,0537 & 63,22 & $-0,2217$ & $-13,30$ \\
\hline Ragusa & 36,923 & 0,9819 & 58,91 & $-0,1498$ & $-8,99$ \\
\hline Ravenna & 44,414 & 0,8131 & 48,79 & $+0,0189$ & $+1,13$ \\
\hline Reggio Calabria & 38,107 & 1,0433 & 62,60 & $-0,2112$ & $-12,67$ \\
\hline Reggio Emilia & 44,698 & 0,7088 & 42,53 & $+0,1232$ & $+7,39$ \\
\hline Rieti & 42,401 & 0,8576 & 51,46 & $-0,0256$ & $-1,53$ \\
\hline Roma (Coll. Rom.) & 41,892 & 0,8321 & 49,93 & 0 & 0 \\
\hline Rovigo & 45,068 & 0,7860 & 47,16 & $+0,0460$ & $+2,76$ \\
\hline Salerno & 40,676 & 0,9835 & 59,01 & $-0,1515$ & $-9,09$ \\
\hline Sassari & 40,730 & 0,5706 & 34,24 & $+0,2614$ & $+15,69$ \\
\hline
\end{tabular}


segue App. D

COORDINATE GRORAFICHE DEI CAPOLUOGH DI PROVINCIA

\begin{tabular}{|c|c|c|c|c|c|}
\hline \multirow{2}{*}{$\begin{array}{c}\text { CAPOLUOGHI } \\
\text { DI } \\
\text { PROVINCIA }\end{array}$} & \multirow{2}{*}{$\begin{array}{c}\text { lat. } N \\
\varphi \\
\text { (gradi) }\end{array}$} & \multicolumn{2}{|c|}{$\begin{array}{l}\text { longitudine } \lambda_{E} \\
\text { Greenwich }\end{array}$} & \multicolumn{2}{|c|}{$\begin{array}{l}\text { longitudine } \lambda \\
\text { da Roma }\left({ }^{* *}\right)\end{array}$} \\
\hline & & (in ore) & (in $\min )$. & (in ore) & (in $\min$.) \\
\hline Savona & 44,302 & 0,5650 & 33,90 & 0,2670 & $+16,02$ \\
\hline Siena & 43,322 & 0,7552 & 45,31 & $+0,0768$ & $+4,61$ \\
\hline Siracusa & 37,072 & 1,0181 & 61,09 & $-0,1861$ & $-11,17$ \\
\hline Sondrio & 46,171 & 0,6578 & 39,47 & $+0,1742$ & $+10,45$ \\
\hline Taranto & 40,468 & 1,1497 & 68,98 & $-0,3176$ & $-19,06$ \\
\hline Teramo & 42,662 & 0,9131 & 54,79 & $-0,0811$ & $-4,87$ \\
\hline Terni & 42,559 & 0,8427 & 50,56 & $-0,0106$ & $-0,64$ \\
\hline Torino & 45.072 & 0,5123 & 30,74 & $+0,3197$ & $+19,18$ \\
\hline Trapani & 38,014 & 0,8349 & 50,09 & $-0,0028$ & $-0,17$ \\
\hline Trento & 46,068 & 0,7416 & 44,50 & $+0,0604$ & $+3,63$ \\
\hline Treviso & 45,669 & 0,8164 & 48,98 & $+0,0156$ & $+0,94$ \\
\hline Trieste & 45,649 & 0,9183 & 55,10 & $-0,0862$ & $-5,17$ \\
\hline Udine & 46,059 & 0,8823 & 52,94 & $-0,0502$ & $-3,01$ \\
\hline Urbino & 43,725 & 0,8442 & 50,54 & $+0,0122$ & $+0,73$ \\
\hline Varese & 45,820 & 0,5883 & 35,30 & $+0,2437$ & $+14,62$ \\
\hline Venezia & 45,432 & 0,8222 & 49,33 & $+0,0098$ & $+0,59$ \\
\hline Vercelli & 45,322 & 0,5612 & 33,67 & $+0,2708$ & $+16,25$ \\
\hline Verona & 45,439 & 0,7329 & 43,98 & $+0,0991$ & $+5,95$ \\
\hline Vicenza & 45,554 & 0,7697 & 46,18 & $+0,0624$ & $+3,74$ \\
\hline Viterbo & 42,415 & 0,8070 & 48,42 & $+0,0250$ & $+1,50$ \\
\hline
\end{tabular}




\begin{tabular}{|c|c|c|c|c|c|}
\hline 兽 & 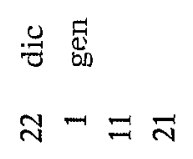 & 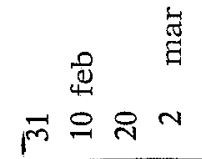 & $\begin{array}{c}\text { 岕 } \\
\approx \approx-=\end{array}$ & 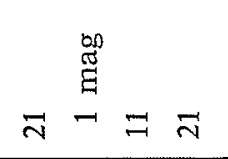 & 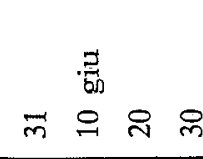 \\
\hline w & 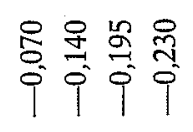 & 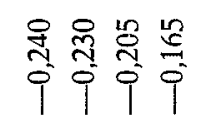 & 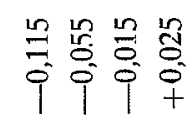 & 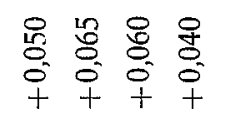 & 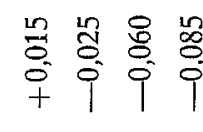 \\
\hline \&̊ำ & 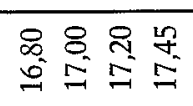 & 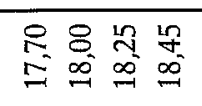 & 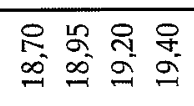 & 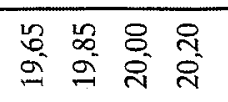 & 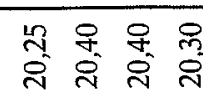 \\
\hline$z^{\stackrel{8}{9}}$ & 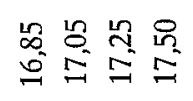 & 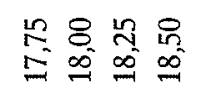 & 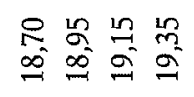 & 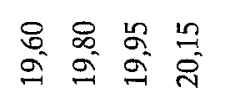 & ్ㅗㄴ \\
\hline 离 & 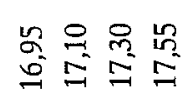 & 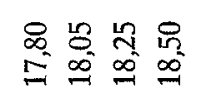 & 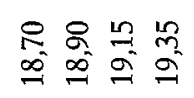 & 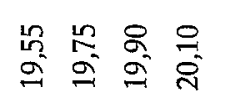 & 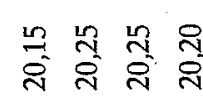 \\
\hline 咅。 & 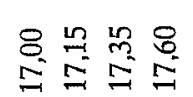 & 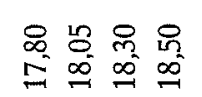 & 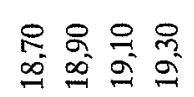 & 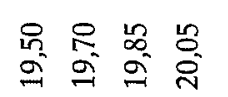 & 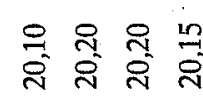 \\
\hline 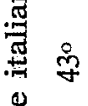 & 总胥导品 & 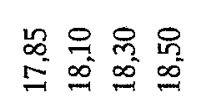 & 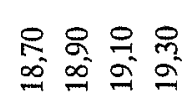 & 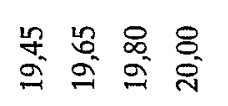 & 옹 \\
\hline 要 & 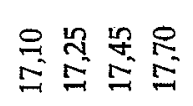 & 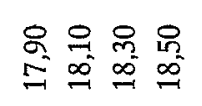 & 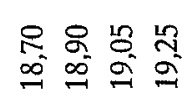 & $\begin{array}{l}9 \\
g \\
g \\
g\end{array}$ & 옴 \\
\hline$\frac{7}{7} \div$ & 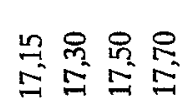 & 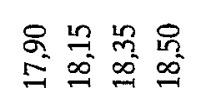 & 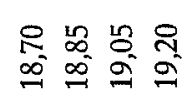 & 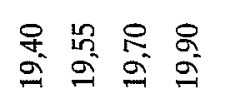 & 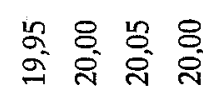 \\
\hline 过 & 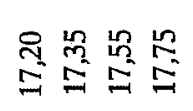 & 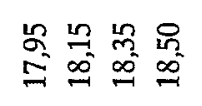 & 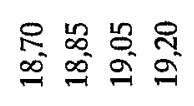 & 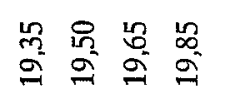 & 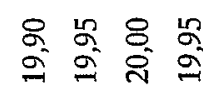 \\
\hline : & 恣是总。 & 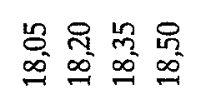 & 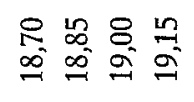 & 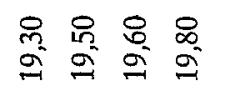 & 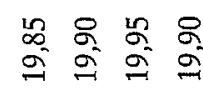 \\
\hline$b$ & 总哭哭哭 & 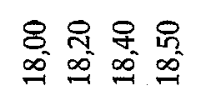 & 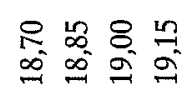 & 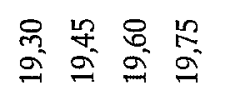 & 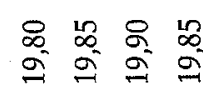 \\
\hline$\stackrel{\circ}{m}$ & 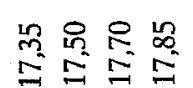 & 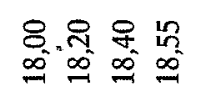 & 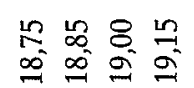 & 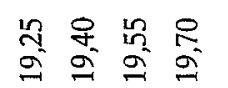 & 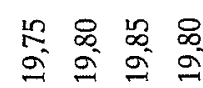 \\
\hline חొ & & 응 Nㅡㄴ & $\tilde{N}-\vec{\pi}$ & $\vec{y}=\vec{N} \vec{m}$ & 의 저 유 으 \\
\hline g & $\dot{8}$ & 递 & 崇 & $\stackrel{g}{g}$ & मे \\
\hline
\end{tabular}




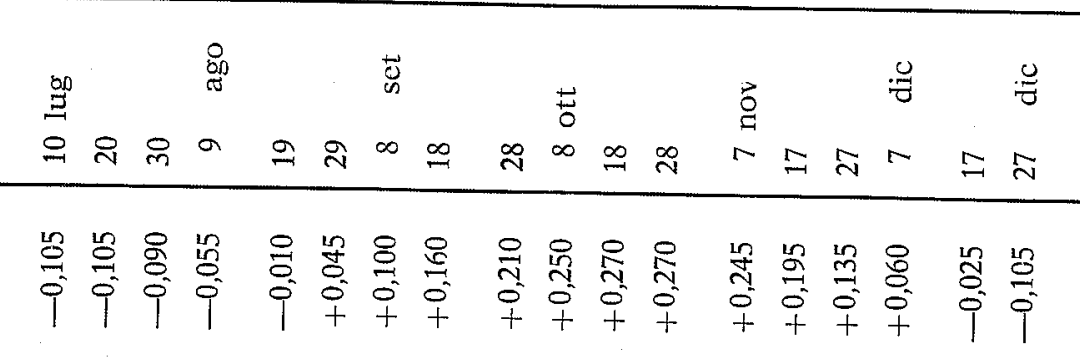

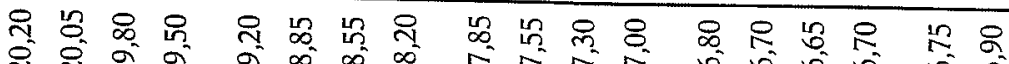

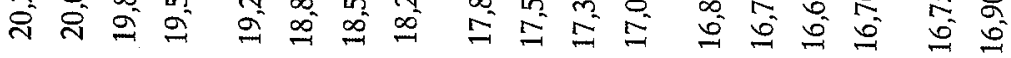

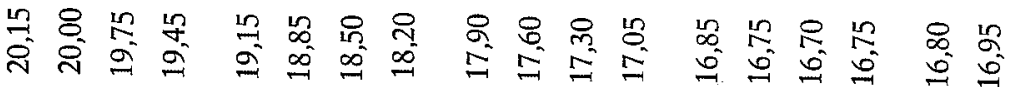

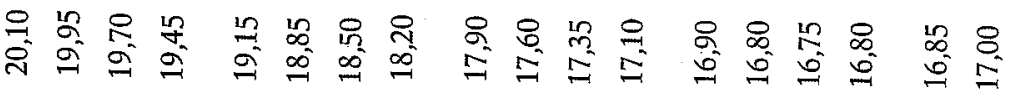

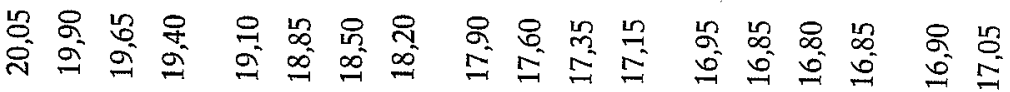

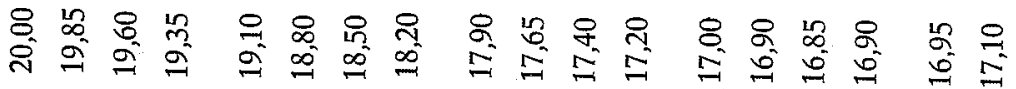

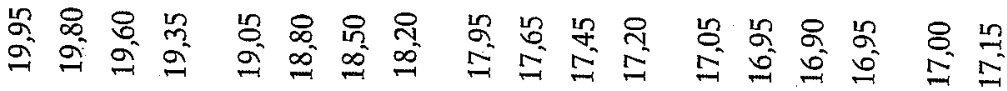

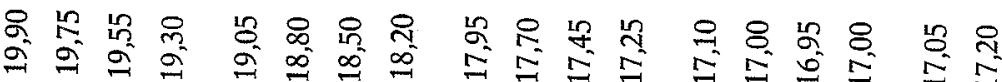

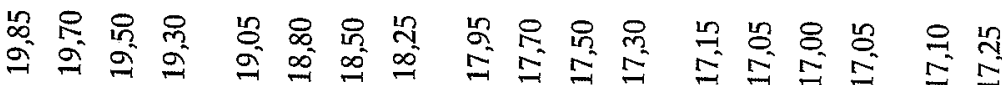

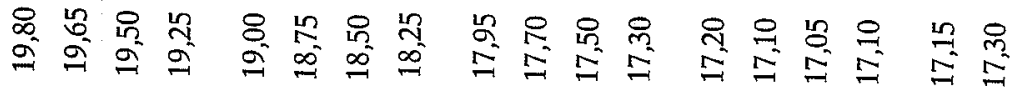
능

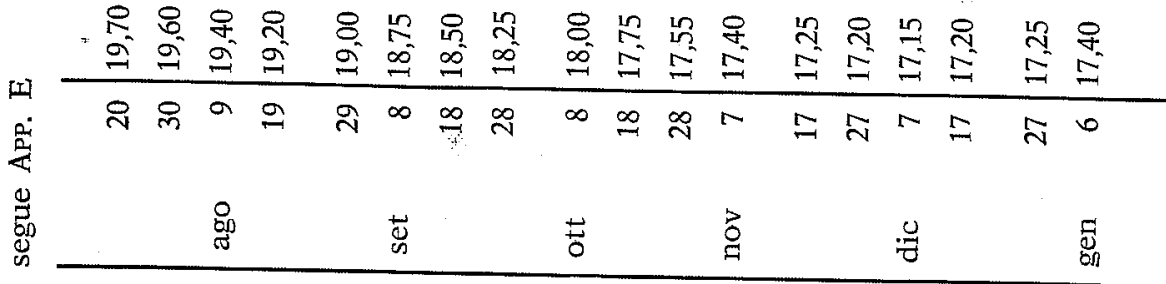


CONClusioni E SINTESI DEI PROCEDIMENTI DA ADOTTARE E DELLE PRECISIONI SUGGERITE PER LE MISURE ORARIE DI EVENTI SISMICI ANTERIORI AL 1894.

Da quanto fin qui esposto risultano con molta chiarezza le difficoltà che si incontrano quando si vogliano tradurre in termini corretti di tempo Greenwich (TU) le notizie di dati orari di eventi storici (o anche soltanto di quelli anteriori all'avvento dei segnali trasmessi via radio): siano esse quelle pervenute a noi dalle fonti originali, siano pure quelle pervenuteci attraverso interpretazioni di scienziati più o meno attenti al problema.

Comunque, poiché sia nell'un caso che nell'altro ci si trova di fronte a incertezze di base generalmente notevoli, riteniamo utile sintetizzare nei passi seguenti il procedimento di massima da seguire per convertire i tempi degli eventi storici in ore attuali (che sono ore civili di tempo medio) e poi in tempi medi (di Roma o) di Greenwich: diamo altresi un quadro degli errori da attribuire alle misure orarie degli eventi storici (non strumentali) secondo i suggerimenti scaturiti dall'analisi critica condotta nel presente lavoro.

Per le notizie orarie date prima del 1400 è da presumere che fossero adottate le ore temporarie (computate da un tramonto all'altro).

Dopo il 1400 entrano nell'uso quasi generale, e perdurano fin verso il 1860, le ore italiane (computate da un'avemaria all'altra).

Sia le une che le altre venivano date in tempo vero.

Il primo passo per la conversione dei tempi in ore attuali va fatto con l'ausilio dell'Appendice $D$ (che dà le coordinate geografiche delle città italiane capoluogo di provincia), dell'Appendice $E$ (che dà il tempo civile medio di inizio del giorno alle varie latitudini geografiche nei vari giorni dell'anno e l'equazione del tempo) e della figura 7 (che dà una sorta di orologio da usare per una rapida conversione approssimativa: una conversione più accurata si ottiene con i calcoli numerici indicati dalle formule [20] e [21].

Particolare attenzione va posta, in questa fase, alla eventualità di un cambiamento di data. 
Ricordiamo che solo in tempi più recenti, intorno al 1816 , cominciano a comparire (ma non generalmente) misure date in tempi medi locali, per cui va posta molta attenzione all'informazione di base che può essere data sia in tempo vero locale (t.v.l.) sia in tempo medio locale (t.m.l.), sia in tempo medio Roma (t.m.R.).

Per avere il tempo dell'evento riferito al meridiano di Roma o a quello di Greenwich, fino al 1894 si può operare come segue:

I) se il tempo dell'evento avvertito nel luogo $A(\varphi, \lambda)$ è dato in t.v.l. (ore temporarie, ore italiane, ore a.m. o p.m.) esso va convertito prima in $t . m . l$., indi in $t . m$. di Roma, poi in TU mediante il seguente procedimento:

$$
\begin{aligned}
\text { t.m.l. } & =\text { t.v.l. }- \text { Eq. tempo } \\
\text { t.m. Roma } & =\text { t.m.l. } \pm \lambda_{\mathrm{A}}{ }^{\text {minuti }}\left({ }^{*}\right)(\mathrm{da} \text { Roma }) \\
& =\text { t.m. Roma }-50^{\mathrm{m}} \\
\mathrm{TU}< & =\text { t.m.l. }-\lambda_{\mathrm{A}}{ }^{\text {minuti }}(\mathrm{da} \text { Greenwich }) .
\end{aligned}
$$

II) se il tempo dell'evento avvertito nel luogo $A$ è dato in tempo medio locale (t.m.l.) vale il processo più rapido:

$$
\begin{aligned}
\text { t.m. Roma } & =t . m . l . \pm \lambda_{\mathrm{A}}{ }^{\text {minuti }}(\mathrm{da} \text { Roma }) \\
& =t . m \text { Roma }-50^{\mathrm{m}} \\
\mathrm{TU}< & \left.=\text { t.m.l. }-\lambda_{\mathrm{A}}{ }^{\text {minuti }} \text { da Greenwich }\right) .
\end{aligned}
$$
Roma.

(*) Vale il segno + se A è a $W$ di Roma, il segno - se A è a $E$ di 
In entrambi i casi va assegnata al TU una attendibilità che viene sintetizzata (in linea di massima) nel modo seguente:

se la misura è data in ore temporarie varrà, dopo la conversione in $\mathrm{TU}$

$$
H=\mathrm{TU} \pm 45^{\mathrm{m}}
$$

(l'errore si può ridurre se si hanno elementi di valutazione più validi); in tutti gli altri casi (ore italiane, ore a.m. o p.m.I varrà:

prima
del
1866 $\begin{cases}H=\mathrm{TU} \pm 30^{\mathrm{m}} & \begin{array}{l}\text { (se la misura originale è data in ore } \\ \text { intere) }\end{array} \\ H=\mathrm{TU}+15^{\mathrm{m}} & \begin{array}{l}\text { (se la misura originale è data al } \\ \text { quarto d'ora) }\end{array}\end{cases}$

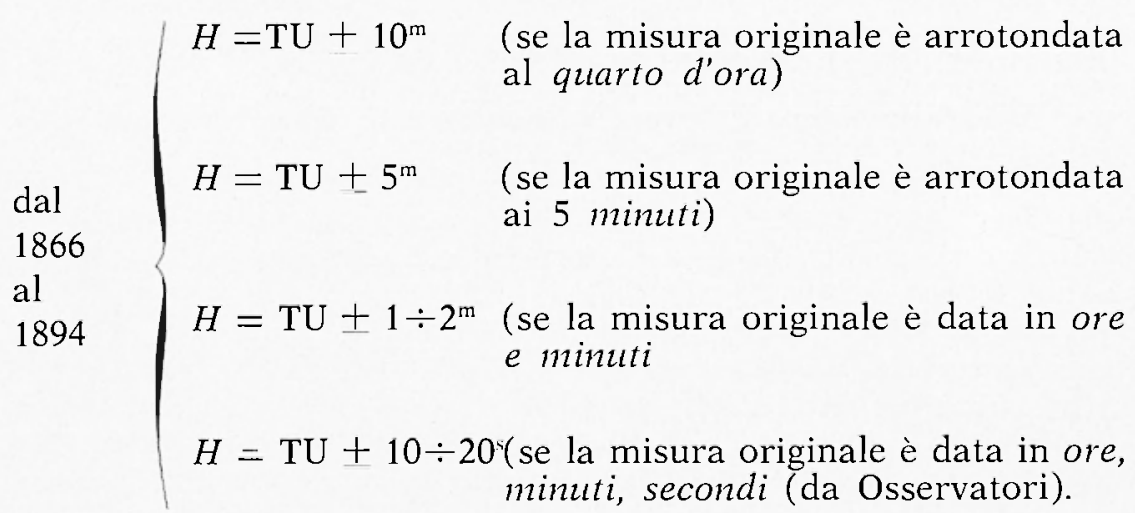

Per gli eventi posteriori al 1894 per lo più si tratta di dati strumentali forniti da Osservatori sismologici, sicché valgono le norme illustrate nel "Preambolo al Catalogo Sismico Nazionale(C.S.N.) I.N.G.».

Va sempre ricordato che i tempi che inizialmente sono riferiti al t.m. di Roma diventano tempi Greenwich (TU) togliendo dal tempo di Roma:

$$
49,8^{\mathrm{m}} \approx 50^{\mathrm{m}}=\lambda_{\text {Roma da Greenwich }}
$$




\section{BIBLIOGRAFIA}

AвEтti G., 1949 - Storia dell'Astronomia, Firenze.

Agrmennone G., 1900-01 - « Boll. Soc. Sism. It. », VI, pag. 45.

Baratta M., 1897 - I terremoti della Liguria e del Piemonte, Napoli.

BARITTA M., 1901 - I terremoti d'Italia, Torino.

Carrozzo M.T., De Visintini G., Giorgetti F., Iaccarino E., 1973 - General Catalogue of Italian Earthquakes, C.N.E.N. RT/PROT(73) 12.

Cavasino A., 1916 - «Boll. Soc. Sism. It. », XX, pag. 11.

De Rossi M.S., 1873 - «Bollettino del vulcanismo italiano » (dal 1873).

Serpieri A., 1889 - Scritti di sismologia (parte II) (raccolti da Giovannozzi).

Serpieri A., 1889 - I terremoti del 18-3-1875 e del 28-7-1883, Firenze.

von BassermanN-JoRdan E. Uhren - Seconda ediz. franc. Fribourg (CH), pag. 372. 